\title{
Le dépôt archaïque du rempart Nord d'Amathonte VII. Autres productions chypriotes et importations levantines
}

\begin{abstract}
Sabine FOURRIER*
RÉSUMÉ Faisant suite aux deux études précédentes, consacrées à des catégories particulières du dépôt (céramiques culinaires et vases du «style d'Amathonte»), cet article s'intéresse à toutes les autres catégories céramiques de production chypriote, ainsi qu’aux importations levantines. Il en dresse le répertoire et propose, de ce fait, une synthèse sur les productions amathousiennes de la fin de l'archaïsme. L'abondant matériel recueilli contre le rempart Nord de la ville basse d'Amathonte mérite, en outre, d'être comparé aux dépôts contemporains de la grotte du sanctuaire d'Aphrodite et des nécropoles : cet assemblage, de composition sensiblement différente, met en évidence l'importance de la pratique du banquet au palais, à la fin de l'époque archaïque.
\end{abstract}

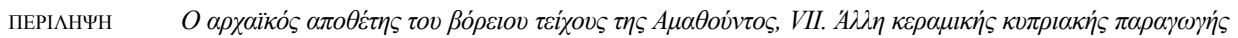

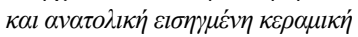

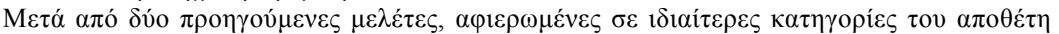

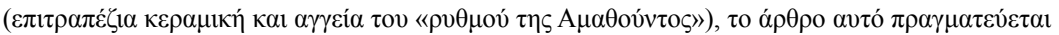

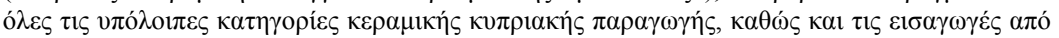

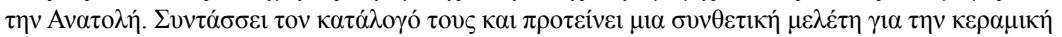

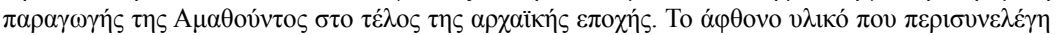

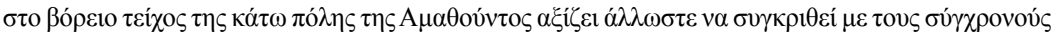

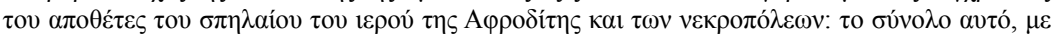

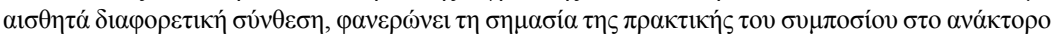

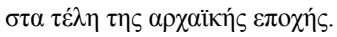

SUMMARY The archaic deposit of the North Rampart at Amathus, VII. Other cypriote wares and levantine imports

After two previous studies, concerning specific categories in the deposit (kitchen wares, and vases of the 'Amathus style'), this article treats all the other ceramic categories of cypriote production, as well as the levantine imports. It draws up the repertoire and thus proposes a synthesis of the amathusian production of late archaic times. The abundant material collected from up against the North Rampart of the Lower Town of Amathus merits, moreover, comparison with the contemporary deposits of the cave sanctuary of Aphrodite and of the cemeteries: this assemblage, of appreciably different composition, stresses the importance of the palatial banquet at the end of the archaic period.

* Déjà publiés dans cette série :

I. «Introduction : le contexte », BCH 127 (2003), p. 101-105.

II. « Les premières inscriptions grecques alphabétiques d'Amathonte (Inscriptions d'Amathonte VI) ", ibid., p. 107-121.

III. « Les petits objets », BCH 128-129 (2004-2005), p. 67-118.

IV. « L'assemblage archéozoologique », BCH 130 (2006), p. 7-35.

V. «Céramiques culinaires », BCH 131 (2007), p. 67-93.

VI. "Vases du "style d'Amathonte” ", BCH 132 (2008), à paraître. 
Je remercie L. Alpe de la grande aide qu'elle m’a apportée lors du tri, de la recherche de raccords et du dénombrement. Tous mes remerciements également à J. Humbert, pour la grande qualité de ses dessins.

Abréviations bibliographiques :

Amathonte VI = S. Fourrier, A. Hermary, Amathonte VI. Le sanctuaire d'Aphrodite des origines au début de l'époque impériale, ÉtChypr XVII (2006).

KB II = J.-Fr. SALLES, Kition-Bamboula II. Les égouts de la ville classique (1983).

Kition VI = V. KarageORghIs, Excavations at Kition VI. The Phoenician and Later Levels (2005).

SCE IV/2 = E. GJERSTAD, The Swedish Cyprus Expedition, IVI2. The Cypro-Geometric, Cypro-Archaic and CyproClassical Periods (1948).

BCH 133 (2009) 


\section{INTRODUCTION}

Sont rassemblées dans cet article toutes les céramiques de production chypriote ou levantine, recueillies dans le dépôt archaïque fouillé contre le rempart Nord d'Amathonte, à l'exception des vases culinaires et du "style d'Amathonte ", qui ont fait l'objet d'études spécifiques dans des tomaisons précédentes ${ }^{1}$. Contrairement à ces deux dernières catégories, le reste de l'abondant matériel mis au jour contre le rempart Nord ne comporte pas de formes inédites ou même rares qui justifie un traitement détaillé. Si la qualité de certains vases ou la présence de certaines formes, inconnues parmi le matériel du sanctuaire de l'acropole, mais généralement documentées par ailleurs, notamment dans les nécropoles, permettent de compléter la typologie de la céramique amathousienne archaïque proposée dans Amathonte VI, l'intérêt de cet ensemble réside principalement dans son contexte. Il s'agit d'un assemblage exceptionnel par sa quantité (qui excède largement les autres dépôts contemporains et conforte donc la validité des conclusions statistiques proposées) et par son origine : sûrement daté du tout début du ve s. av. J.-C. et probablement lié aux destructions causées par le siège d'Onésilos de Salamine, ce matériel provient du palais de l'acropole ${ }^{2}$. Il s'agit donc du seul ensemble clos, connu pour le moment, qui reflète le mobilier céramique d'un palais de rois chypriotes à l'époque archaïque. Or, ce matériel, qui est directement comparable à celui qui était déposé dans le sanctuaire de l'acropole et qui a été étudié selon la même méthode de dénombrement, offre une composition sensiblement différente, et dans les formes et dans les fabriques. Ce constat permet de réviser certains classements de la typologie proposée par E. Gjerstad (SCE IV/2), de réfléchir aux usages possibles de cette céramique et, partant, de s'interroger sur les activités qui avaient lieu au palais à l'époque archaïque.

La démarche suivie a été la même que pour l'étude des deux dépôts archaïques du sanctuaire d'Aphrodite : je renvoie donc à Amathonte VI pour l'exposé de la méthode, qui repose sur un dénombrement précis ${ }^{3}$, le mode de présentation de la typologie et les abré-

1. Les importations grecques, qui représentent un lot important, par leur qualité, leur variété (elles comprennent à la fois des amphores commerciales et des céramiques fines) et leur nombre, remarquable si on le compare à la rareté des importations égéennes dans les dépôts contemporains du sanctuaire, seront publiées par B. Blandin. Pour significatives qu'elles soient, elles ne constituent toutefois qu'une part infime de l'abondant matériel jeté contre la pente pour étayer le rempart Nord d'époque classique.

2. Voir B. Blandin, S. Fourrier, BCH 127 (2003), p. 101-105.

3. Amathonte VI, p. 49-126 ; voir en particulier p. 50 pour les distorsions qui peuvent apparaitre dans les tableaux statistiques. Même si le matériel a été trié au moment de la fouille lors des premières années d'exploration du dépôt et même si ce dernier n'a pas été vidé en totalité, pour des raisons de sécurité, le lot recueilli en 2001 est suffisamment abondant pour être jugé représentatif de l'ensemble (voir BCH 126 [2002], p. 571-576). Les céramiques culinaires et les vases du "style d'Amathonte ", qui ont été étudiés à part, ont été intégrés au dénombrement général, de même que certaines céramiques publiées avec les petits objets (BCH 128-129 [2004-2005], p. 67-118) : seul l'ostracon AM 1944, les lampes et les ratés de cuisson, qui ne sont pas ou plus des vases, et les importations grecques, qui sont en cours d'étude, n'apparaissent pas dans les tableaux généraux.

BCH 133 (2009) 
viations utilisées. Seuls les types nouveaux, qui ne sont pas attestés parmi le matériel publié du sanctuaire, feront l'objet d'une étude détaillée, avec l'indication d'éventuels parallèles : on se reportera, pour les autres, à la typologie établie dans Amathonte VI, dont on suit l'ordre, avec quelques aménagements mineurs. Comme dans cette dernière publication, un vase représentatif, appelé " tête de série ", illustre, autant que faire se peut, chaque forme attestée dans chaque fabrique. Son numéro d'inventaire, qui renvoie au classement typologique, facilite la comparaison avec le matériel du sanctuaire ${ }^{4}$. Les tableaux statistiques généraux comprennent une référence à l'assemblage céramique de la grotte du sanctuaire d'Aphrodite, qui est un dépôt déposé à la même date que celui du rempart Nord (fin de la période Chypro-Archaïque II) et donc le seul à lui être directement comparable.

\section{LE RÉPERTOIRE MORPHOLOGIQUE}

\section{1. FORMES OUVERTES}

\begin{tabular}{l|r|r|r|c}
\multirow{2}{*}{ Type } & \multicolumn{2}{|c|}{ Rempart Nord } & \multicolumn{2}{c}{ Grotte } \\
\cline { 2 - 5 } & NMI & \multicolumn{1}{c}{$\%$} & NMI & \% \\
\hline \hline A.1.1 & 55 & $2,2 \%$ & 68 & $3,4 \%$ \\
\hline A.1.2 & 30 & $1,2 \%$ & 32 & $1,6 \%$ \\
\hline A.1.3 & 1038 & $41,5 \%$ & 54 & $2,7 \%$ \\
\hline Total A.1. & 1123 & $\mathbf{4 4 , 9} \%$ & $\mathbf{1 5 8}$ & $7,9 \%$ \\
\hline A.2.1 & 601 & $24 \%$ & 272 & $13,7 \%$ \\
\hline A.2.5 & 80 & $3,2 \%$ & 12 & $0,6 \%$ \\
\hline Total A.2 & $\mathbf{6 8 1}$ & $\mathbf{2 7 , 2} \%$ & $\mathbf{2 9 7}$ & $\mathbf{1 5} \%$ \\
\hline A.3.2 & 55 & $2,2 \%$ & 62 & $3,1 \%$ \\
\hline A.4.1 & 22 & $0,9 \%$ & 0 & $0 \%$ \\
\hline A.4.2 & 317 & $12,7 \%$ & 225 & $11,3 \%$ \\
\hline Total A.4 & 339 & $\mathbf{1 3 , 6} \%$ & $\mathbf{2 2 5}$ & $\mathbf{1 1 , 3} \%$ \\
\hline TOTAL A & $\mathbf{2 1 9 8}$ & $\mathbf{8 7 , 9} \%$ & $\mathbf{8 1 9}$ & $\mathbf{4 1 , 2} \%$ \\
\hline B.2.1 & 47 & $1,9 \%$ & 202 & $10,2 \%$ \\
\hline B.2.2 & 8 & $0,3 \%$ & 283 & $14,2 \%$ \\
\hline Total B.2 & 55 & $\mathbf{2 , 2} \%$ & 494 & $\mathbf{2 4 , 9} \%$ \\
\hline
\end{tabular}

4. Ainsi, un numéro d'inventaire du type « $\mathrm{d} / \mathrm{a} \cdot \mathrm{bich} r .1$ » indique le contexte ( $\mathrm{d}$ " pour « dépôt du rempart Nord "), la forme (" a " pour " amphore »), la fabrique (" bichr. " pour «Bichrome ») et le no au sein de la série («1»). Seuls les objets inventoriés individuellement au musée de Limassol possèdent une numérotation différente, du type $\mathrm{AM}+\mathrm{n}^{\circ}$. La qualité des dessins a permis de réduire la description des têtes de série au strict minimum.

BCH 133 (2009) 


\begin{tabular}{l|c|c|c|c}
\multirow{2}{*}{ Type } & \multicolumn{2}{|c|}{ Rempart Nord } & \multicolumn{2}{c}{ Grotte } \\
\cline { 2 - 5 } & NMI & $\mathbf{\%}$ & NMI & \% \\
\hline \hline B.3.2 & 51 & $2,1 \%$ & 63 & $3,2 \%$ \\
\hline B.4.2 & 8 & $0,3 \%$ & 31 & $1,6 \%$ \\
\hline B.5.2 & 2 & $0,1 \%$ & 10 & $0,5 \%$ \\
\hline B.6.1 & 40 & $1,6 \%$ & 2 & $0,1 \%$ \\
\hline TOTAL B & $\mathbf{1 5 6}$ & $\mathbf{6 , 3} \%$ & $\mathbf{6 5 9}$ & $\mathbf{3 3 , 2} \%$ \\
\hline C.1.1 & 133 & $5,3 \%$ & 498 & $25,1 \%$ \\
\hline C.1.3 & 10 & $0,4 \%$ & 11 & $0,5 \%$ \\
\hline C.2.2 & 3 & $0,1 \%$ & 0 & $0 \%$ \\
\hline TOTAL C & $\mathbf{1 4 6}$ & $\mathbf{5 , 8} \%$ & $\mathbf{5 0 9}$ & $\mathbf{2 5 , 6} \%$ \\
\hline TOTAL & $\mathbf{2 5 0 0}$ & $\mathbf{1 0 0} \%$ & $\mathbf{1 9 8 7}$ & $\mathbf{1 0 0} \%$ \\
\hline \hline
\end{tabular}

Tableau 1. - Répartition des formes ouvertes. Comparaison entre les dépôts du rempart Nord et de la grotte du sanctuaire.

\begin{tabular}{|c|c|c|c|c|c|c|c|c|c|}
\hline Type & PW & WP & Bichr. & $\mathbf{R S}$ & BS & BoR & \begin{tabular}{|c|} 
Bichr.R. et \\
«blackened $»$
\end{tabular} & Total & $\%$ \\
\hline A.1.1 & 8 & 10 & 0 & 19 & 7 & 0 & 11 & 55 & $1,9 \%$ \\
\hline A.1.2 & 1 & 0 & 0 & 16 & 0 & 13 & 0 & 30 & $1 \%$ \\
\hline A.1.3 & 167 & 238 & 35 & 0 & 0 & 111 & 487 & 1038 & $34,7 \%$ \\
\hline Total A.1 & 176 & 248 & 35 & 35 & 7 & 124 & 498 & 1123 & $37,6 \%$ \\
\hline A.2.1 & 596 & 3 & 2 & 0 & 0 & 0 & 0 & 601 & $20,1 \%$ \\
\hline A. 2.5 & 30 & 2 & 6 & 29 & 7 & 4 & 2 & 80 & $2,7 \%$ \\
\hline Total A.2 & 626 & 5 & 8 & 29 & 7 & 4 & 2 & 681 & $22,8 \%$ \\
\hline A.3.2 & 6 & 9 & 40 & 0 & 0 & 0 & 0 & 55 & $1,9 \%$ \\
\hline A. 4.1 & 22 & 0 & 0 & 0 & 0 & 0 & 0 & 22 & $0,7 \%$ \\
\hline A. 4.2 & 188 & 27 & 90 & 9 & 0 & 3 & 0 & 317 & $10,6 \%$ \\
\hline Total A.4 & 210 & 27 & 90 & 9 & 0 & 3 & 0 & 339 & $11,3 \%$ \\
\hline TOTAL A & 1018 & 289 & 173 & 73 & 14 & 131 & 500 & 2198 & $73,6 \%$ \\
\hline B.2.1 & 0 & 0 & 22 & $1 ?$ & $3 ?$ & 10 & 11 & 47 & $1,6 \%$ \\
\hline B.2.2 & 1 & $1 ?$ & 6 & 0 & 0 & 0 & 0 & 8 & $0,2 \%$ \\
\hline Total B.2 & 1 & 1 & 28 & 1 & 3 & 10 & 11 & 55 & $1,8 \%$ \\
\hline B.3.2 & 22 & 29 & 0 & 0 & 0 & 0 & 0 & 51 & $1,7 \%$ \\
\hline B.4.2 & 0 & 5 & 0 & 3 & 0 & 0 & 0 & 8 & $0,3 \%$ \\
\hline B.5.2 & & & 2 & 0 & 0 & 0 & 0 & 2 & $0,1 \%$ \\
\hline
\end{tabular}




\begin{tabular}{|c|c|c|c|c|c|c|c|c|c|}
\hline Type & PW & WP & Bichr. & RS & BS & BoR & 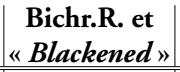 & Total & $\%$ \\
\hline B.6.1 & 6 & 0 & 3 & 4 & 1 & 8 & 18 & 40 & $1,3 \%$ \\
\hline TOTAL B & 29 & 35 & 33 & 8 & 4 & 18 & 29 & 156 & $5,2 \%$ \\
\hline C.1.1 & 128 & 0 & 0 & 4 & 0 & 1 & 0 & 133 & $4,5 \%$ \\
\hline C.1.3 & 3 & 0 & 4 & 3 & 0 & 0 & 0 & 10 & $0,3 \%$ \\
\hline C.2.2 & 0 & 0 & 3 & 0 & 0 & 0 & 0 & 3 & $0,1 \%$ \\
\hline TOTAL C & 131 & $\mathbf{0}$ & 7 & 7 & $\mathbf{0}$ & 1 & $\mathbf{0}$ & 146 & $4,9 \%$ \\
\hline D. 1 & 12 & 0 & 0 & 0 & 0 & 0 & 0 & 12 & $0,4 \%$ \\
\hline D. 2 & 1 & 0 & 0 & 0 & 0 & 0 & 0 & 1 & $0 \%$ \\
\hline D.3 & \begin{tabular}{|}
475 (dont \\
12 D. 3.1 , \\
3 D.3.2 et \\
9 D. 3.3 ) \\
\end{tabular} & 0 & 0 & 0 & 0 & 0 & 0 & 475 & $15,9 \%$ \\
\hline TOTAL D & 488 & $\mathbf{0}$ & $\mathbf{0}$ & $\mathbf{0}$ & $\mathbf{0}$ & $\mathbf{0}$ & $\mathbf{0}$ & 488 & $16,3 \%$ \\
\hline TOTAL & 1666 & 324 & 213 & 88 & 18 & 150 & 529 & 2988 & $100 \%$ \\
\hline$\%$ & $55,8 \%$ & $10,8 \%$ & $7,1 \%$ & $3 \%$ & $0,6 \%$ & $5 \%$ & $17,7 \%$ & $100 \%$ & \\
\hline
\end{tabular}

Tableau 2. - Répartition des formes ouvertes par type et par fabrique.

A. BOL PEU PROFOND À LARGE OUVERTURE (fig. 1-35)

A.1. Épaule marquée, rebord vertical, légèrement évasé ou légèrement rentrant (fig. 1-16)

A.1.1. Lèvre ronde (fig. 1-3)

Têtes de série : d/b.pw.10, d/b.rs.1, d/b.bs.1.

A.1.2. Lèvre à léger biseau interne (fig. 4-6)

Têtes de série : d/b.wp.4, d/b.rs.2, d/b.bor.1.

A.1.3. Lèvre à net biseau interne (fig. 7-16)

Têtes de série : d/b.pw.17, d/b.wp.5, d/b.bichr.3, d/b.bichr.5, d/b.bor.2, d/b.bichr.r.2, d/b.bichr.r.5, d/b.bichr.r.6, d/b.bs.4, d/b.bs.6.

Les vases recueillis dans le dépôt du rempart Nord possèdent des traits morphologiques (épaule très marquée, rebord généralement rentrant) qui trahissent un type évolué, à placer dans la classe $\mathrm{V}$. La prépondérance des lèvres à net biseau interne offre également un indice chronologique et participe de la même évolution des formes : le pincement qui façonne la lèvre s'accompagne d'une pression sur la paroi qui accentue la rupture de l'épaule. Certains exemplaires, de module plus réduit et réalisés dans des fabriques sans décor (PW, RS, BS), sont dépourvus d'anse (ainsi d/b.bs.1, fig. 3), tandis que les bols décorés sont 
systématiquement pourvus de petites anses en ruban ${ }^{5}$. Les bols de fabrique Bichr.R. et Bichr.R. "Blackened", qui sont les plus nombreux, illustrent parfaitement les tendances de la production amathousienne à la fin de la période archaïque : goût pour des formes de dimensions souvent réduites et des schémas décoratifs chargés, répétitifs et colorés.

\section{A.2. Profil de courbe continue (fig. 17-25)}

A.2.1. Bol de faible hauteur, rebord vertical, légèrement évasé ou rentrant, lèvre ronde (fig. 17-20)

Têtes de série : d/b.pw.22, d/b.pw.26, d/b.wp.7.

A.2.5. Parois évasées, lèvre nettement étalée (fig. 21-25)

Têtes de série : $\mathrm{d} / \mathrm{b}$. bichr.8, $\mathrm{d} / \mathrm{b}$. bor.3.

Variante à ressaut interne sous la lèvre: $\mathrm{d} / \mathrm{b}$.pw.4, $\mathrm{d} / \mathrm{b} . \mathrm{rs} .4, \mathrm{~d} / \mathrm{b} . \mathrm{bs} .2$.

Le type A.2.1 est très fréquent en fabrique PW (fig. 20). Cela conforte les conclusions suggérées dans Amathonte VI : ce type est caractéristique de l'archaïsme tardif, ce que confirment à la fois les dimensions des bols (certains sont même miniatures) et la forme de la lèvre, parfois nettement rentrante. Les bols, à fond plat ou plat saillant, sont dépourvus d'anse. Certains rares exemplaires ont une vasque plus profonde, qui invite à les placer dans la catégorie des bols de type B, mais il m'a paru peu satisfaisant de scinder en deux une série si homogène.

Les types A.2.2, A.2.3 et A.2.4, marginaux dans le dépôt de la grotte, sont complètement absents du remplissage contemporain du rempart Nord.

Le type A.2.5 est peu attesté. Les bols à lèvre étalée simple ne sont sûrement illustrés qu'en fabrique Bichr. et BoR. Les exemples dénombrés en fabriques WP et Bichr.R. ne sont représentés que par de petits fragments de lèvre, trop incomplets pour que l'identification soit assurée. En revanche, la variante à lèvre à ressaut interne, inconnue dans les dépôts du sanctuaire, est documentée par plusieurs exemplaires, tous réalisés dans des fabriques sans décor peint, PW et RS. La forme se rapproche de celle des plats à marli de production ou d'inspiration levantine, particulièrement fréquents dans les niveaux du Chypro-Archaïque II et du Chypro-Classique de Kition ${ }^{6}$. Un certain nombre d'exemplaires du rempart Nord possèdent une pâte fine qui n'est pas amathousienne (de production levantine ou kitienne).

5. Elles ne sont toutefois représentées sur les dessins que si elles sont, entièrement ou partiellement, conservées sur le vase.

6. Ce type de bol à lèvre à encoche apparaît, sous forme d'importations ou d'imitations fidèles en RS, dès les niveaux de la période archaïque I à Kition (voir, par exemple, Kition VI, pl. CXXVII 2749). Au cours du Chypro-Archaïque II, les bols, le plus souvent en fabrique PW, sont de hauteur plus réduite et les parois ne sont plus de profil rectiligne évasé (ibid., pl. CLXXII 5244). P. Bikai classe ces plats à marli parmi ses " common ware plates ", qui proviennent pour la plupart des fouilles de Kition-Kathari: P. BIKAI, The Phoenician Pottery of Cyprus (1987), p. 41-43, pl. XX.

BCH 133 (2009) 
A.3. Profil caréné (fig. 26-28)

A.3.2. Bol à parois nettement évasées, lèvre pendante (fig. 26-28)

Têtes de série : d/b.pw.5, d/b.wp.13, d/b.bichr.23.

Seul le type à parois nettement évasées et lèvre pendante est attesté. Il s'agit d'une évolution du type dérivé des céramiques fines levantines, dites parfois "Samaria Ware ${ }^{7}$ ". Le profil écrasé, la position basse de la carène sont des indices morphologiques caractéristiques de la classe V. Comme dans les autres types, les séries miniatures sont nombreuses, en particulier dans les fabriques décorées (Bichr., fig. 28). Elles signent l'une des grandes tendances stylistiques du répertoire amathousien de la fin de l'archaïsme. Ce type n'existe que dans les fabriques à surface claire, engobée ou non (PW, WP et Bichr.).

\section{A.4. Profil évasé rectiligne (fig. 29-35)}

A.4.1. Lèvre ronde ou légèrement biseautée (fig. 29)

Tête de série : $\mathrm{d} / \mathrm{b}$.pw.14.

Ce type, qui n'avait été identifié que grâce à un petit bol Bichr. du sanctuaire, est représenté par une vingtaine d'individus provenant du dépôt du rempart Nord. L'origine levantine de la forme est probable ${ }^{8}$, ce que confirme l'emploi, en l'occurrence exclusif, d'une fabrique sans décor $(\mathrm{PW})$. Les parois très inclinées invitent à placer cette série de production dans la classe V. Les bols à parois évasées rectilignes de la classe IV ont généralement une vasque plus profonde et une lèvre étalée 9 .

\section{A.4.2. Lèvre étalée (fig. 30-35)}

Têtes de série : d/b.pw.19, d/b.pw.21, d/b.wp.10, d/b.bichr.12, d/b.bichr.14, d/b.rs.5.

Les bols de fabriques décorées (WP et Bichr.) sont de plus grandes dimensions que les bols PW ou RS. Ils possèdent un décor chargé et très coloré. Certains exemplaires monochromes sont munis d'une lèvre longue, marquée d'un net ressaut interne, à l'imitation des plats à marli d'origine levantine (voir supra le commentaire du type A.2.5).

B. BOL DE PROFONDEUR MOYENNE (fig. 36-54)

B.2. Profil de courbe continue ${ }^{10}$ (fig. 36-43)

7. Amathonte VI, p. 55.

8. Voir les références données dans Amathonte VI, p. 56, n. 40.

9. Voir, par exemple, P. Flourentzos, RDAC 2004, pl. XIV 124(28) et 124(29) : bols RS II(IV) de la Tombe 646 d'Amathonte.

10. Le type à épaule marquée (B.1), plus ancien, n’est pas attesté parmi le matériel du rempart Nord : voir Amathonte VI, p. 57-58.

BCH 133 (2009) 
B.2.1. Lèvre ronde ou amincie (fig. 36-41)

Têtes de série : d/b.bichr.17, d/b.bichr.19, d/b.bor.5, d/b.bichr.r.11, d/b.bs.7.

La dizaine d'individus en fabrique BoR (fig. 39) sont très fragmentaires et ils correspondent probablement à un matériel résiduel, datable de la classe IV (certains fragments appartiennent peut-être à des bols Bichr.R., que l'état de conservation des tessons ne permet pas d'identifier sûrement). En revanche, la série Bichr. présente toutes les caractéristiques d'une production amathousienne de la fin de l'archaïsme : les parois des bols sont nettement convexes, les dimensions petites, le décor, avec ses larges croisillons et ses frises de motifs géométriques et végétaux superposés (fig. 38), n’est pas sans rappeler celui des vases du "style d'Amathonte ", qui est cependant rarement décliné sur des formes ouvertes ${ }^{11}$. Les bols Bichr.R. et Bichr.R. "Blackened», qui portent un décor similaire, en constituent le pendant parmi les productions à engobe rouge.

B.2.2. Lèvre à biseau (fig. 42-43)

Têtes de série : d/b.pw.18, d/b.bichr.20.

Le type est marginal parmi les bols découverts dans le dépôt du rempart Nord. Il est totalement absent dans les productions à engobe rouge.

\section{B.3. Profil caréné (fig. 44-45)}

Ce type est l'équivalent, parmi les bols profonds, du type A.3. Comme pour ce dernier, seul le type évolué de la fin de l'archaïsme (B.3.2) est attesté dans le matériel du rempart Nord.

B.3.2. Lèvre étalée pendante (fig. 44-45)

Têtes de série : d/b.wp.8, d/b.bichr.10.

B.4. Corps de courbe complexe, à parois convexes et rebord évasé (fig. 46-47)

Le type B.4.1, à épaule marquée, est caractéristique de la classe IV ${ }^{12}$. Il n'est pas représenté parmi le matériel, plus tardif, du dépôt du rempart Nord.

B.4.2. Profil de courbe continue (fig. 46-47)

Têtes de série : d/b.wp.15, d/b.rs.6.

La série de petits bols sans anses, à fond plat saillant, et frise de feuilles sur le haut de la vasque (fig. 46) manque de parallèle. Le motif décoratif est emprunté aux vases du "style d'Amathonte ", ce qui indique qu'il s'agit d'une production locale, caractéristique de la fin de l'archaïsme.

11. Voir la série du rempart Nord, publiée dans le BCH 132 (2008), p. 555-585.

12. Amathonte VI, p. 60. 


\section{B.5. Bol miniature à ouverture étroite (fig. 48)}

Cette forme, qui n'apparaît que de manière anecdotique parmi les dépôts archaïques d'Amathonte, quel qu'en soit le contexte, est illustrée par très peu d'exemplaires, tous à corps de profil biconvexe (B.5.2) et à décor de bandes Bichr., autant de traits caractéristiques des productions de la fin de l'archaïsme.

B.5.2. Corps de profil biconvexe (fig. 48)

Tête de série : d/b.bichr.25.

\section{B.6. Bol à lèvre (fig. 49-54)}

Seul le type B.6.1 est sûrement attesté, ce qui ne saurait surprendre : le type B.6.2, à large rebord légèrement évasé et ressaut sous la lèvre, est surtout en vogue au ChyproGéométrique III et Chypro-Archaïque I et il est le plus souvent décliné en fabrique BoR ${ }^{13}$. Les quelques bols à lèvre du rempart Nord sont de module réduit, leur ligne de profil est "écrasée » et, lorsqu'ils sont peints, ils portent un riche décor bariolé, autant de traits caractéristiques de la fin de l'archaïsme. On notera que tous les exemplaires dont le profil est à peu près complet sont dépourvus d'anse, à l'exception d'un seul bol, de plus grandes dimensions (d/b.bichr.r.9, fig. 54), qui est muni d'anses horizontales redressées, très décollées de la paroi.

B.6.1. Rebord vertical et épaule marquée (fig. 49-54)

Têtes de série : d/b.pw.1, d/b.bichr.26, d/b.rs.7, d/b.bor.7, d/b.bichr.r.9, d/b.bichr.r.10.

\section{BOL À PAROI FINE (fig. 55-58)}

Cette série de production, qui reprend une forme de vase à boire caractéristique du Levant, apparaît en grand nombre dans les niveaux archaïques du sanctuaire d'Aphrodite à Amathonte ${ }^{14}$. Les exemplaires du dépôt du rempart Nord sont proportionnellement moins nombreux et ils entrent majoritairement, comme ceux de la grotte, dans le type du bol profond de profil convexe (type C.1.1). Le type C.1.2, caractéristique des niveaux du Chypro-Archaïque I et qui est très bien représenté, à Amathonte, parmi le matériel du bothros, est totalement absent. Autre indice d'une datation à la fin de la période archaïque, une surface entièrement réservée remplace le décor de bande pourpre sur le haut de la vasque. Les quelques bols à parois évasées (type C.1.3, fig. 57) ou de format miniature et à panse carénée (type C.2.2, fig. 58) portent ce décor de bande et sont sans doute à interpréter comme matériel résiduel dans des niveaux plus récents.

13. Ibid., p. 61.

14. Ibid., p. 61-63.

BCH 133 (2009) 


\section{C.1. Bol profond}

C.1.1. Profil convexe (fig. 55-56)

Têtes de série : $\mathrm{d} / \mathrm{b} \cdot \mathrm{pw} \cdot 6, \mathrm{~d} / \mathrm{b} \cdot \mathrm{pw} .7$.

C.1.3. Parois nettement évasées (fig. 57)

Tête de série : d/b.bichr.27.

\section{C.2. Bol miniature}

\section{C.2.2. Profil caréné (fig. 58)}

Tête de série : d/b.bichr.28.

\section{Cuvettes (fig. 59-65)}

Alors que cette catégorie céramique n'est que marginalement représentée parmi le matériel du sanctuaire d'Aphrodite ${ }^{15}$, et uniquement par des fragments de " bols perses ", les vases du dépôt du rempart Nord sont plus nombreux et, surtout, ils appartiennent à des types variés. La place importante occupée au palais par ces céramiques destinées à préparer la nourriture — ou éventuellement à la distribuer — recoupe celle des céramiques à feu, destinées à la cuire ${ }^{16}$. Encore une fois, les assemblages de vases indiquent que le palais était un lieu de transformation, de préparation et de cuisson des aliments, que l'on doit mettre en relation avec la pratique du banquet collectif.

Seuls les « bols perses ", dans leurs deux variantes principales (à fond plat/à base en anneau), constituent des séries abondantes. Il en est de même pour les céramiques culinaires, qui offrent une grande variété de types, mais parmi lesquelles les marmites prédominent largement. On remarquera que ces deux catégories, auxquelles il faut ajouter les amphores à anses de panier, sont également caractéristiques des niveaux classiques mis au jour lors de la fouille du palais de l'acropole ${ }^{17}$.

15. Ibid., p. 63.

16. Pour l'interprétation des « bols perses ", voir les indices bibliographiques donnés dans Amathonte VI, p. 63. Pour leurs centres de production, voir les récentes analyses publiées par M. SPATARO, A. VILLING, "Scientific Investigation of Pottery Grinding Bowls from the Archaic and Classical Eastern Mediterranean ", The British Museum Technical Research Bulletin 3 (2010), p. 89-100. Pour l'assemblage de céramiques culinaires du dépôt du rempart Nord, S. FOURRIER, BCH 131 (2007), p. 67-93.

17. Pour les amphores à anses de panier, voir Th. Petit, dans Cl. BaUrain, C. Bonnet, V. Krings (éds), Phoinikeia Grammata, Studia Phoenicia XII (1991), p. 481-490. Les « bols perses » sont également l'une des catégories céramiques les plus nombreuses parmi le matériel de la fin de l'époque classique, découvert dans le port d'Amathonte : voir Fr. ALABE, La céramique non amphorique sub-classique du port d'Amathonte, mémoire inédit de 3e année de l'EFA (1988).

BCH 133 (2009) 
D.1. A parois épaisses et rebord peu élevé, lèvre rentrante (fig. 59)

Tête de série : $\mathrm{d} / \mathrm{b} \cdot \mathrm{pw} \cdot 15$ (fig. 59a).

Ce type est, à ma connaissance, sans parallèle. Il n'est pas sûr qu'il s'agisse d'un récipient : la courbure peu prononcée de la paroi invite à restituer une forme de grandes dimensions, dont l'ouverture n'est pas circulaire. Des fragments de forme voisine, inédits, ont été recueillis dans les niveaux archaïques du sanctuaire de Kition-Bamboula, dans une zone artisanale (fig. 59b-c) : il pourrait s'agir de fragments de soufflets. L'absence de marques de feu, sur les fragments de Kition, indique que ces éléments céramiques n'étaient pas en contact direct ni avec la flamme, ni avec le métal en fusion. Mais la pâte des exemplaires kitiens, ferrugineuse et réfractaire, montre cependant une proximité avec le feu, dont ne témoigne pas la pâte calcaire des quelques tessons d'Amathonte ${ }^{18}$. La présence d'éventuels ateliers métallurgiques dans le palais, si toutefois cette interprétation est juste, ne saurait surprendre ${ }^{19}$.

\section{D.2. À parois épaisses, évasées, rebord à biseau externe (fig. 60)}

Tête de série: $\mathrm{d} / \mathrm{b} . p w .16$.

Comp. : Amathonte : Fr. VAndenabeELE, BCH 109 (1985), p. 645, fig. 63.

Kition-Bamboula: KB II, p. 71, fig. 28, 246.

Le type n'est illustré que par un seul exemplaire, qui se distingue en outre par un traitement de surface particulier (incisions dans la pâte avant cuisson, faites au doigt ou à l'aide d'un instrument large). La forme est banale, mais les parallèles sont rares. L'exemple d'Amathonte provient du remplissage d'un silo et il est daté de la période archaïque. Celui de Kition a été recueilli dans l'égout 113 (le matériel qui y a été découvert date des environs du milieu du IVe s. av. J.-C.). Ce dernier récipient se distingue, par de nombreux détails, de la cuvette du rempart Nord : ses parois ont une courbure plus prononcée, sa lèvre est moulurée, mais on notera un mode de décor voisin, né de l'impression du doigt dans la pâte.

\section{D.3. «Bol perse » (fig. 61-65)}

La quantité et la variété des exemplaires recueillis dans le dépôt du rempart Nord permettent de compléter la typologie proposée dans Amathonte VI, sans en remettre en question les conclusions chronologiques : les cuvettes de ce type sont aussi bien munies de

18. Un fragment du sanctuaire de Kition-Kathari que V. Karageorghis interprète comme un plateau ou un bassin, entre peut-être également dans cette catégorie. Même s'il provient de niveaux post-classiques non stratifiés, on remarquera qu'il a été découvert avec divers fragments métalliques : Kition VI, pl. CI et CLXXXVIII 1439. Mentionnons également un autre récipient fragmentaire, recueilli dans les niveaux classiques de Kition-Bamboula (locus $D$ ) et que le fouilleur interprète comme un creuset en terre cuite : $K B$ II, p. 105, fig. 39, 383. Ce dernier exemple possède toutefois des parois plus élevées.

19. Sur l'existence d'ateliers dans le palais, dont témoigne le matériel, voir les références données par S. FOURRIER, BCH 128-129 (2004-2005), p. 102.

BCH 133 (2009) 
fond plat que de base en anneau, sans que ce détail morphologique ait une signification chronologique ${ }^{20}$. En l'absence de raccord assuré, il est difficile de classer les fragments de «bols perses ». Toutes les lèvres qu'on n'a pas pu rattacher à un type de fond précis sont en bourrelet externe (451 individus différents). Seuls les bols dont l'ouverture est déformée en panier (type D.3.3) possèdent une lèvre ronde, dans le prolongement de la paroi. D'autres détails, notamment la présence d'une anse verticale, distinguent d'ailleurs cette dernière variante. Il m'a toutefois paru préférable de la faire entrer dans la catégorie des "bols perses ", dont elle possède les traits morphologiques principaux.

Les « bols perses ", communs dans les niveaux classiques du palais d'Amathonte, sont relativement rares parmi les céramiques des tombes contemporaines. Ils entrent toutefois dans la composition du matériel funéraire de tombes d'autres régions, à Idalion, Salamine ou Aphendrika ${ }^{21}$. Les riches dépôts céramiques de Kafizin documentent, pour une période plus récente, une grande variété de grands bols, cuvettes, ou mortiers, qu'il est tentant de mettre en relation avec une dîme, versée au sanctuaire de la Nymphe ${ }^{22}$. Mais le parallèle le plus révélateur est fourni par les nécropoles de Salamine, où les "bols perses » apparaissent, dès la période archaïque, dans la composition des offrandes des « tombes royales » ${ }^{23}$. Comme je l'ai suggéré ailleurs, ces dernières offrent une image synthétique du mobilier palatial, elles en mettent en scène les types caractéristiques : on ne saurait donc être surpris de l'association de chars et de meubles en matières précieuses avec des céramiques culinaires et des cuvettes ${ }^{24}$. On n'a pas encore découvert de tombes "royales " à Amathonte, mais on peut supposer qu'elles livreraient un matériel similaire : malgré des spécificités culturelles marquées, les royaumes chypriotes partagent un certain nombre de pratiques et de symboles royaux que l'on peut qualifier de " panchypriotes » 25 .

20. On a dénombré 84 fragments de fonds plats et 59 fragments de bases en anneau plat. Seul le type à fond plat est recensé dans la typologie suédoise : SCE IV/2, fig. LVI 23.

21. Idalion : V. KarageOrghis, RDAC 1964, p. 69, fig. 23. Cette tombe (Tombe 2) est datée du ChyproClassique I. Salamine : V. KARAGEORGHIS, Excavations in the Necropolis of Salamis II (1970), pl. LIII 3 et 9 (Tombe 6, datée du Chypro-Archaïque II) ; pl. LXV 85 (Tombe 10, utilisée au cours des périodes archaïque et classique) ; pl. LXXXV 5, 7, 13 et 14 (Tombe 18, Chypro-Classique I), etc. Aphendrika : E. Dray, J. Du Plat TaYlor, RDAC 1937-1939, p. 89, fig. 42 (tombes classiques).

22. T. B. MitForD, The Nymphaeum of Kafizin, Kadmos Suppl. 2 (1980), p. 44-154. Malheureusement, seul le matériel inscrit est pour le moment publié, et l'on ne dispose d'aucune indication statistique sur la composition typologique des offrandes.

23. V. KARAGEORGHIS, Excavations in the Necropolis of Salamis I (1967), pl. CXI (Tombe 2, datée du ChyproArchaïque I) ; pl. XLI et CXXV (Tombe 3, datée des environs de 600 av. J.-C.) ; pl. CXLIX (Tombe 50, inhumation datée du Chypro-Archaïque I-II) ; id., Excavations in the Necropolis of Salamis III (1973), pl. CCXXV et CCXXXIII (Tombe 79, datée du Chypro-Archaïque I).

24. S. FourRIER, BCH 131 (2007), p. 81.

25. Il est probable que le sarcophage d'Amathonte de la collection Cesnola (V. KARAGEORGHIS et al., Ancient Art from Cyprus. The Cesnola Collection in the Metropolitan Museum of Art [2000], p. 201-204, cat. no 330) provient d'une tombe « royale». Malheureusement, le matériel qui lui était associé est perdu et

BCH 133 (2009) 
D.3.1. À fond plat (fig. 61-62)

Têtes de série : d/b.pw.27, d/b.pw.28.

D.3.2. À base en anneau plat (fig. 63-64)

Têtes de série $: \mathrm{d} / \mathrm{b} . p w .32, \mathrm{~d} / \mathrm{b} \cdot \mathrm{pw} .33$.

D.3.3. À ouverture en panier (fig. 65)

Tête de série : $\mathrm{d} / \mathrm{b} \cdot \mathrm{pw} .31$.

Dans cette dernière variante morphologique, qui est documentée par neuf individus, la cuvette est munie d'une anse verticale et l'ouverture est étirée en une forme ovale plutôt que circulaire. C'est là un trait rare, mais récurrent, dans la production chypriote depuis le début de l'époque géométrique et le type possède des parallèles, plus ou moins proches, dans diverses fabriques, peintes ou non ${ }^{26}$. Il est probable qu'il s'agit de l'imitation, en terre cuite, d'une forme de panier en matière périssable.

\section{2. CRUCheS}

\begin{tabular}{|c|c|c|c|c|}
\hline \multirow[t]{2}{*}{ Type } & \multicolumn{2}{|c|}{ Rempart Nord } & \multicolumn{2}{|l|}{ Grotte } \\
\hline & NMI & $\%$ & NMI & $\%$ \\
\hline A. 1.1 & 311 & $51,6 \%$ & 439 & $69 \%$ \\
\hline A.1.2 & 4 & $0,6 \%$ & 0 & $0 \%$ \\
\hline Total A.1 & $315+62$ ind. $=377$ & $62,5 \%$ & 439 & $69 \%$ \\
\hline A. 2.2 & 1 & $0,2 \%$ & 0 & $0 \%$ \\
\hline A.3 & 10 & $1,6 \%$ & 4 & $0,6 \%$ \\
\hline A. 4 & 49 & $8,1 \%$ & 0 & $0 \%$ \\
\hline TOTAL A & $437+7$ ind. $=444$ & $73,6 \%$ & 443 & $69,6 \%$ \\
\hline B.1.1 & 82 & $13,6 \%$ & 111 & $17,4 \%$ \\
\hline B.1.2 & 71 & $11,8 \%$ & 0 & $0 \%$ \\
\hline Total B.1 & 153 & $25,4 \%$ & $111+55$ ind. $=166$ & $26,1 \%$ \\
\hline B.2.1 & 1 & $0,2 \%$ & 1 & $0,2 \%$ \\
\hline
\end{tabular}

on n'a aucun renseignement fiable sur le contexte de découverte. Sur l'idéologie royale panchypriote, voir S. FourRier, dans R. ÉTIENNE (dir.), La Méditerranée au VII siècle av. J.-C. (2010), p. 164-165.

26. Comparer, par exemple, avec V. Karageorghis, Palaepaphos-Skales. An Iron Age Cemetery in Cyprus (1983), fig. LVI 74 (tombe datée du début du chypro-Géométrique I), K. NicolaOU, RDAC 1964, pl. XVII 7 (tombe chypro-archaïque I de Marion), SCE IV/2, fig. XLVIII 1 (bol Bichr. Và décor figuré du British Museum) et G. WalBerG, RDAC 1979, p. 276-280 (bol Bichr. V du musée d'Uppsala). Plusieurs bols PW des tombes « royales » archaïques de Salamine présentent la même déformation de l'ouverture, mais la forme générale du bol, à vasque plus profonde, est différente : V. KARAGEORGHIS (1967, supra, n. 23), pl. CXXXIII 82 et 59 (Tombe 31, datée du Chypro-Archaïque I).

BCH 133 (2009) 


\begin{tabular}{l|r|r|r|r} 
Type & \multicolumn{3}{|c}{ Rempart Nord } & \multicolumn{2}{c}{ Grotte } \\
\hline \hline TOTAL B & $\mathbf{1 5 4}$ & $\mathbf{2 5 , 6} \%$ & $\mathbf{1 6 7}$ & $\mathbf{2 6 , 3} \%$ \\
\hline C.1 & 2 & $0,3 \%$ & 0 & $0 \%$ \\
\hline C.2.2 & 2 & $0,3 \%$ & 6 & $1 \%$ \\
\hline C.2.4 & 0 & $0 \%$ & 7 & $1,1 \%$ \\
\hline Total C.2 & 2 & $\mathbf{0 , 3} \%$ & 13 & $\mathbf{2 , 1} \%$ \\
\hline C.3 & 0 & $0 \%$ & 9 & $1,4 \%$ \\
\hline TOTAL C & $\mathbf{4}$ & $\mathbf{0 , 6} \%$ & $\mathbf{2 2}$ & $\mathbf{3 , 5} \%$ \\
\hline D & $\mathbf{1}$ (pondéré) & $\mathbf{0 , 2} \%$ & 4 & $\mathbf{0 , 6} \%$ \\
\hline TOTAL & $\mathbf{6 0 3}$ & $\mathbf{1 0 0} \%$ & $\mathbf{6 3 6}$ & $\mathbf{1 0 0} \%$ \\
\hline \hline
\end{tabular}

Tableau 3. - Répartition des cruches. Comparaison entre les dépôts du rempart Nord et de la grotte du sanctuaire.

\begin{tabular}{|c|c|c|c|c|c|c|c|c|c|}
\hline Type & PW & WP & Bichr. & RS & BS & BoR & $\left|\begin{array}{c}\text { Bichr.R. et } \\
\text { «Blackened }\end{array}\right|$ & Total & $\%$ \\
\hline A.1.1 & 158 & 0 & 0 & 53 & 100 & 0 & 0 & 311 & $51,6 \%$ \\
\hline A. 1.2 & 4 & 0 & 0 & 0 & 0 & 0 & 0 & 4 & $0,6 \%$ \\
\hline Total A.1 & $\begin{array}{r}162+62 \\
\text { ind. }=224\end{array}$ & 0 & 0 & 53 & 100 & 0 & 0 & 377 & $62,5 \%$ \\
\hline A. 2.2 & 0 & 1 & 0 & 0 & 0 & 0 & 0 & 1 & $0,2 \%$ \\
\hline A.3 & 3 & 0 & 1 & 0 & 4 & 2 & 0 & 10 & $1,6 \%$ \\
\hline A. 4 & 43 & 0 & 0 & 3 & 3 & 0 & 0 & 49 & $8,1 \%$ \\
\hline TOTAL A & $\begin{array}{r}270+7 \\
\text { ind. }=277\end{array}$ & 1 & 1 & 56 & 107 & 2 & 0 & 444 & $73,6 \%$ \\
\hline B.1.1 & 9 & 28 & 34 & 2 & 0 & 9 & 0 & 82 & $13,6 \%$ \\
\hline B.1.2 & 44 & 26 & 1 & 0 & 0 & 0 & 0 & 71 & $11,8 \%$ \\
\hline Total B.1 & 53 & 54 & 35 & 2 & 0 & 9 & 0 & 153 & $25,4 \%$ \\
\hline B.2.1 & 0 & 1 & 0 & 0 & 0 & 0 & 0 & 1 & $0,2 \%$ \\
\hline TOTAL B & 53 & 55 & 35 & 2 & $\mathbf{0}$ & 9 & $\mathbf{0}$ & 154 & $25,6 \%$ \\
\hline C.1 & 0 & 0 & 2 & 0 & 0 & 0 & 0 & 2 & $0,3 \%$ \\
\hline C. 2.2 & 0 & 0 & 0 & 0 & 0 & 2 & 0 & 2 & $0,3 \%$ \\
\hline TOTAL C & $\mathbf{0}$ & 0 & 2 & 0 & 0 & 2 & 0 & 4 & $0,6 \%$ \\
\hline $\mathrm{D}$ & 1 (pondéré) & 0 & 0 & 0 & 0 & 0 & 0 & 1 & $0,2 \%$ \\
\hline TOTAL & 331 & 56 & 38 & 58 & 107 & 13 & 0 & 603 & $100 \%$ \\
\hline$\%$ & $54,9 \%$ & $9,3 \%$ & $6,3 \%$ & $9,6 \%$ & $17,7 \%$ & $2,2 \%$ & $0 \%$ & $100 \%$ & \\
\hline
\end{tabular}

Tableau 4. - Répartition des cruches par type et par fabrique. 
A. CRUChe À OUVERTURe RONDE (fig. 66-81)

\section{A.1. Col cylindrique ou légèrement évasé, lèvre ronde (fig. 66-73)}

A.1.1. Corps globulaire (fig. 66-71)

Têtes de série : d/cr.pw.1, d/cr.rs.9, d/cr.rs.6, d/cr.rs.3, d/cr.bs.1, d/cr.bs.6.

La forme est courante dès l'époque géométrique et elle présente peu de variations au cours de l'Âge du Fer. Certains traits morphologiques soulignent toutefois la date récente des cruches du dépôt du rempart Nord : le col a tendance à être plus étroit, resserré à sa base ; beaucoup de cruches sont de petit format, parfois même miniatures (fig. 69); le corps est de profil très globulaire, voire biconique pour les exemplaires les plus petits. Ce type n'existe que dans des fabriques sans décor peint, à surface engobée ou non.

La variante à attache supérieure de l'anse sur le col, qui n'était attestée que marginalement dans le sanctuaire et uniquement dans le dépôt de la grotte, est documenté par 34 exemplaires du rempart Nord (neuf de fabrique RS et vingt-cinq de fabrique BS). De même, en raison de leur petit format, les cruches à base annulaire (fig. 70) sont beaucoup plus rares que celles à fond plat saillant.

A.1.2. Corps étroit (fig. 72-73)

Têtes de série : AM 2624, AM 2628.

Le type n'est sûrement documenté que par 4 exemplaires de fabrique PW. Il s'agit, là encore, d'une forme connue de l'époque géométrique à l'époque classique et qui, isolée, est de datation difficile. Certes, les cruches de ce type paraissent moins nombreuses dans des contextes du Chypro-Archaïque II que dans ceux du début de l'époque archaïque, mais elles n'y sont pas complètement inconnues ${ }^{27}$ et le type est à nouveau très en vogue à l'époque classique.

\section{A.2. Col cylindrique étroit, lèvre ronde (fig. 74)}

A.2.2. Anse du rebord à l'épaule (fig. 74)

Tête de série : $\mathrm{d} /$ cr.wp. 1 .

Comp. : ?

Cette forme de cruche n'était attestée dans le sanctuaire que par un exemplaire à attache de l'anse sur le col, datable du Chypro-Archaïque I ${ }^{28}$. Elle est également isolée, dans sa variante à attache de l'anse sur le rebord, dans le dépôt du rempart Nord.

27. Voir, par exemple, un exemplaire recueilli dans la Tombe 251 de la nécropole Ouest d'Amathonte : Th. PETIT, RDAC 2007, p. 207, pl. V.13. Dans les assemblages du sanctuaire, le type est absent du dépôt de la grotte, alors qu'il est documenté par 32 individus du bothros: Amathonte VI, p. 88, tableau 3.

28. Amathonte VI, p. 65.

BCH 133 (2009) 
Selon une autre lecture du fragment, il pourrait s'agir d'une cruche à bec (type D.2), qui possède des dimensions réduites et un profil de col voisin ${ }^{29}$. Mais l'absence de tessons de becs en fabriques peintes et l'état de conservation du fragment interdisent toute conclusion.

A.3. Col cylindrique étroit, lèvre étalée, anse de la lèvre à l'épaule (fig. 75-78)

Têtes de série :d/cr.pw.5, d/cr.bichr.4, d/cr.bs.3, d/cr.bor.3.

La lèvre pendante, le profil biconique de certains exemples du rempart Nord invitent à les dater de l'extrême fin de la période archaïque ${ }^{30}$.

A.4. Col cylindrique étroit, évasé vers le haut, anse de la lèvre à l'épaule, débordante (fig. 79-81)

Têtes de série : $\mathrm{d} /$ cr.pw.4, d/cr.rs.4, d/cr.bs.4.

Comp.: Amathonte (palais) : $\quad$ BCH 102 (1978), p. 949, fig. 17 (PW VI).

Amathonte (nécropoles) : $B C H 84$ (1960), p. 267, fig. 39 (WP VI).

P. Flourentzos, RDAC 2004, pl. I, 9 (PW V) ; pl. II, 12 et 13 (RS III[V]) ; pl. III, 30 (PW V) ; pl. IV, 31 (RS III[V]) et 32 (PW V) ; pl. VI, 60 (RS III[V]) et 61 (PW V) ; pl. VII, 62 (RS III[V]) ; pl. XX, 124(90) (RS III[V]), 124(92) (RS III[V]), 124(93) (PW V), 124(94) (PW V), 124(96) (RS III[V]), 124(97) (Bichr.R.II[IV] sic) ; pl. XXI, 124(98) (RS III[V]) et 124(99) (RS III[V]).

I. NicOlaOU, $R D A C 1985$, p. 268, fig. 6, 11 et 12 (PW VI) ; pl. XLVI, 32 (PW VI) et 34 (WP VI) ; pl. XLVII, 54 et 56a (WP VI) ; pl. XLVIII, 91 (PW VI), 92 (Bichr.R. III[VI]) et 116 (WP VI).

Th. PETIT, RDAC 2007, pl. VII, 22-23 (« White on Red VI »).

Chr. TytGat, $B C H 103$ (1979), p. 743, fig. 14.

Kornos : $\quad$ M. LoulLOUPIS, RDAC 1967, pl. XXV, 52 (Bichr.R. II[V]).

Limassol : $\quad$ BCH 86 (1962), p. 379, fig. 73, centre (WP VI).

BCH 128-129 (2004-2005), p. 1652, 39 (Bichr.R. II[V]).

P. FlourENTZOS, RDAC 1993, pl. XXXV, 1, 12 (Bichr.R. III[VI]) et 6 (PW VI) ; pl. XXXVI, 16 (PW VI) ; pl. XXXVII, 29, 34 (Bichr.R. III[VI]) et 32 (PW VI) ; pl. XL, 67 (Bichr.R. III[VI]).

Pyrgos : $\quad$ BCH91 (1967), p. 308, fig. 93 (Bichr.R. II[V]).

29. Voir, par exemple, $B C H 126$ (2002), p. 703, fig. 49 (cruche-biberon de la Tombe 783 de la nécropole Est d'Amathonte, qu'on attribuera plus volontiers à la classe V).

30. Aux parallèles proposés dans Amathonte VI, p. 65-66, on peut ajouter Kition VI, pl. CLIII 3764, qui provient d'un niveau daté des sols 2A-2, soit la fin du Chypro-Archaïque II/début du Chypro-Classique I.

BCH 133 (2009) 
Ce type n'avait pas été répertorié parmi les cruches découvertes dans le sanctuaire ${ }^{31}$. La forme est cependant commune dans les tombes contemporaines de la fin de l'époque archaïque et les parallèles ne manquent pas. Elle n'est attestée qu'à Amathonte et sur le territoire du royaume (tombes de Limassol, Pyrgos et Kornos) : il s'agit d'une production originale, propre aux ateliers amathousiens. On peut noter quelques variantes dans le détail de la morphologie, notamment dans la forme de la lèvre, simplement ronde dans le prolongement du rebord évasé ou repliée et légèrement incurvée vers l'intérieur. De même, l'anse peut être en ruban ou bifide et elle tend à déborder largement au-dessus de l'horizontale de l'ouverture. Toutes les cruches de ce type, découvertes dans le dépôt du rempart Nord, sont dépourvues de décor peint (fabriques PW, RS et BS). Les exemples des nécropoles montrent cependant que la forme peut être déclinée en fabrique WP ou Bichr. (avec souvent seulement une bande rouge sur la panse, sans peinture noire associée, mais aussi parfois des motifs empruntés au "style d'Amathonte»). Ils indiquent également que les cruches RS (et "Blackened») portent généralement une large bande de peinture blanche sur la panse (Th. Petit propose de dénommer la fabrique "White on Red»). L'attribution du type à la classe VI, proposée par E. Gjerstad, me paraît préférable à une attribution à la classe $\mathrm{V}$, parfois suggérée. En effet, même si cette forme est aussi attestée dans des contextes purement archaïques et non pas seulement du Chypro-Classique I, il s'agit toujours d'assemblages de la fin du Chypro-Archaïque II et les caractéristiques générales de ce type (anse débordante, profil écrasé, col de plus en plus étroit, lèvre de plus en plus volumineuse et repliée, décor simplifié ou surface entièrement réservée) renvoient au répertoire de la période classique.

\section{B. CRUCHE À OUVERTURE PINCÉE (fig. 82-93)}

\section{B.1. Col cylindrique et corps globulaire (fig. 82-92)}

\section{B.1.1. Col haut et étroit (fig. 82-89)}

Têtes de série : d/cr.pw.8, d/cr.pw.9, d/cr.wp.2, d/cr.bichr.5, d/cr.bichr.7, d/cr.bichr.9, d/cr.rs.1, d/cr.bor.1.

Deux tendances morphologiques indiquent la date récente des exemplaires du dépôt du rempart Nord : la lèvre devient plus haute par rapport à la hauteur générale du col, au point de parfois se confondre avec lui ${ }^{32}$; les vases sont souvent de dimensions réduites, ce qui écrase le profil de la panse. Une même date à la fin de l'archaïsme est suggérée par le décor d'œil qui orne la lèvre : c'est un motif déjà connu dans la classe céramique précé-

31. Il est illustré dans la classe VI de la typologie suédoise par des cruches du British Museum : SCE IV/2, fig. LVIII 7 (WP VI) ; fig. LIX 6 (Bichr. VI) ; fig. LX 16 (Bichr.R. III[VI]).

32. C'est, dans la typologie suédoise, une caractéristique des classes $\mathrm{V}$ et $\mathrm{VI}$ : voir, par exemple, SCE IV/2, fig. LVII 14 (PW V) et fig. LX 18 (Bichr.R. III[VI]).

BCH 133 (2009) 
dente, notamment sur les cruches en Free Field Style (Amathonte VI, type B.2.1), mais il connaît une vogue particulière dans la fabrique Bichr. V33.

B.1.2. Col plus court et plus large (fig. 90-92)

Têtes de série : $\mathrm{d} /$ cr.pw.11, d/cr.wp.5, d/cr.bichr.8.

Dans cette variante encore, les détails de la lèvre, épaissie et peu distincte du col, et le choix de certaines fabriques, comme de la technique du «Bichrome amathousien " ${ }^{34}$, invitent à placer les cruches dans la classe V. Le type n'est pas attesté parmi le matériel de la grotte, et il n'est documenté au sanctuaire que par quelques exemplaires du bothros, tous attribuables à la classe IV 35 .

\section{B.2. Col court et évasé (fig. 93)}

\section{B.2.1. Corps biconvexe (fig. 93)}

Tête de série : $\mathrm{d} /$ cr.wp.3.

Cette série de production typiquement amathousienne est caractéristique de la classe IV ${ }^{36}$. L'exemplaire du rempart Nord est évidemment résiduel.

C. CRUCHe À RESSAUt SUR Le COl ANSE DU COl À l'épaule (fig. 94-95)

C.1. Lèvre ronde ou en léger bourrelet externe (fig. 94)

Tête de série : $\mathrm{d} /$ cr.bichr.2.

Le type n'est représenté que par deux fragments Bichr. du Rempart Nord, sans doute résiduels.

\section{C.2. Lèvre étalée (fig. 95)}

C.2.2. Cruche de petite taille, à col évasé et corps globulaire (fig. 95)

Tête de série : $\mathrm{d} /$ cr.bor.4.

Comme le précédent, ce type est bien documenté dans la classe IV. Les deux exemples du rempart Nord, de fabrique BoR, sont résiduels.

D. CRUCHE À BEC (fig. 96)

33. SCE IV/2, fig. XLIX 6, 9 et 11-13. Pour l'ensemble des cruches de type B.1 du dépôt du rempart Nord, voir également les parallèles de la Tombe 251 de la nécropole d'Amathonte, datée de la fin du ChyproArchaïque II : Th. PeTIT, RDAC 2007, p. 193-210.

34. Pour l'identification de cette fabrique, voir Fl. DANIEL et al., BCH 131 (2007), p. 44-45.

35. Amathonte VI, p. 67.

36. Ibid., p. 67-69. 
L'existence de cruches à bec n'est assurée que grâce à un fragment PW (d/cr.pw.7) qu'il est impossible d'identifier plus précisément.

\section{3. AMPHORES}

\begin{tabular}{|c|c|c|c|c|}
\hline \multirow[t]{2}{*}{ Type } & \multicolumn{2}{|c|}{ Rempart Nord } & \multicolumn{2}{|c|}{ Grotte } \\
\hline & NMI & $\%$ & NMI & $\%$ \\
\hline A. 1 & 18 & $1,4 \%$ & 20 & $3,4 \%$ \\
\hline A.2.1 & 1 & $0,1 \%$ & 1 & $0,2 \%$ \\
\hline A. 2.2 & 1 & $0,1 \%$ & 20 & $3,4 \%$ \\
\hline A. 2.3 & 16 & $1,2 \%$ & 16 & $2,7 \%$ \\
\hline A.2.4 & 0 & $0 \%$ & 2 & $0,3 \%$ \\
\hline TOTAL A & 36 & $2,8 \%$ & 60 & $10,2 \%$ \\
\hline B.1.1 & 2 & $0,1 \%$ & 2 & $0,3 \%$ \\
\hline B.2.1 & 159 & $12,2 \%$ & 90 & $15,2 \%$ \\
\hline B.2.3 & 2 & $0,1 \%$ & 0 & $0 \%$ \\
\hline B.3 & 236 & $18,2 \%$ & 307 & $51,9 \%$ \\
\hline B.4.1 & 557 & $42,8 \%$ & 94 & $15,9 \%$ \\
\hline B.4.2 & 4 & $0,3 \%$ & 0 & $0 \%$ \\
\hline B.5 & 9 & $0,7 \%$ & 33 & $5,6 \%$ \\
\hline TOTAL B & 969 & $74,4 \%$ & 526 & $88,9 \%$ \\
\hline C.1 & 77 & $5,9 \%$ & 3 & $0,5 \%$ \\
\hline C.2 & 0 & $0 \%$ & 1 & $0,2 \%$ \\
\hline C.3 & 219 & $16,8 \%$ & 0 & $0 \%$ \\
\hline TOTAL C & 296 & $22,7 \%$ & 4 & $0,7 \%$ \\
\hline $\mathrm{D}$ & 1 (pondéré) & $0,1 \%$ & 1 (pondéré) & $0,2 \%$ \\
\hline TOTAL & 1302 & $100 \%$ & 591 & $100 \%$ \\
\hline
\end{tabular}

Tableau 5. - Répartition des amphores. Comparaison entre les dépôts du rempart Nord et de la grotte du sanctuaire.

\begin{tabular}{l|r|r|r|r|r|r|r|r|r} 
Type & PW & WP & Bichr. & RS & BS & BoR & $\begin{array}{c}\text { Bichr.R. et } \\
\text { «lackened }\end{array}$ & Total & \% \\
\hline \hline A.1 & 3 & 7 & 7 & 0 & 0 & 0 & 1 (pondéré) & 18 & $1,4 \%$ \\
\hline A.2.1 & 0 & 0 & 0 & 0 & 0 & 1 & 0 & 1 & $0,1 \%$ \\
\hline A.2.2 & 1 & 0 & 0 & 0 & 0 & 0 & 0 & 1 & $0,1 \%$ \\
\hline A.2.3 & 0 & 0 & 16 & 0 & 0 & 0 & 0 & 16 & $1,2 \%$ \\
\hline
\end{tabular}

BCH 133 (2009) 


\begin{tabular}{|c|c|c|c|c|c|c|c|c|c|}
\hline Type & PW & WP & Bichr. & $\mathbf{R S}$ & BS & BoR & \begin{tabular}{|c} 
Bichr.R. et \\
«Blackened»
\end{tabular} & Total & $\%$ \\
\hline TOTAL A & 4 & 7 & 23 & 0 & 0 & 1 & 1 & 36 & $2,8 \%$ \\
\hline B.1.1 & 0 & 0 & 0 & 0 & 0 & 1 & 1 & 2 & $0,1 \%$ \\
\hline B.2.1 & 52 & 106 & 0 & 0 & 0 & 0 & 1 & 159 & $12,2 \%$ \\
\hline B.2.3 & 2 & 0 & 0 & 0 & 0 & 0 & 0 & 2 & $0,1 \%$ \\
\hline B.3 & 29 & 41 & 125 & 0 & 0 & 0 & 41 & 236 & $18,2 \%$ \\
\hline B.4.1 & 125 & 273 & 126 & 0 & 0 & 8 & 25 & 557 & $42,8 \%$ \\
\hline B.4.2 & 0 & 0 & 0 & 0 & 0 & 0 & 4 & 4 & $0,3 \%$ \\
\hline B. 5 & 1 (pondéré) & 0 & 8 & 0 & 0 & 0 & 0 & 9 & $0,7 \%$ \\
\hline TOTAL B & 209 & 420 & 259 & 0 & 0 & 9 & 72 & 969 & $74,4 \%$ \\
\hline C. 1 & 77 & 0 & 0 & 0 & 0 & 0 & 0 & 77 & $5,9 \%$ \\
\hline C. 3 & 219 & 0 & 0 & 0 & 0 & 0 & 0 & 219 & $16,8 \%$ \\
\hline TOTAL C & 296 & 0 & 0 & 0 & 0 & 0 & 0 & 296 & $22,7 \%$ \\
\hline D & 1 (pondéré) & 0 & 0 & 0 & 0 & 0 & 0 & 1 & $0,1 \%$ \\
\hline TOTAL & 510 & 427 & 282 & $\mathbf{0}$ & $\mathbf{0}$ & 10 & 73 & 1302 & $100 \%$ \\
\hline$\%$ & $39,2 \%$ & $32,8 \%$ & $21,6 \%$ & $0 \%$ & $0 \%$ & $0,8 \%$ & $5,6 \%$ & $100 \%$ & \\
\hline
\end{tabular}

Tableau 6. - Répartition des amphores par type et par fabrique.

\section{A. AMPHORE A ANSES VerTiCALES (fig. 97-103)}

A.1. Amphore de grande taille, lèvre en bourrelet externe, anse du col à l'épaule (fig. 97-105)

Têtes de série : d/a.pw.14, d/a.pw.18, d/a.wp.1, d/a.wp.2, d/a.bichr.2, d/a.bichr.1, d/a.bichr.3, d/a. bichr.4.

Les dimensions de ces amphores les rapprochent de la catégorie des pithoi, bien attestés dans les magasins du palais classique ${ }^{37}$. Le type, que caractérise un col court et large sur lequel est appliquée l'attache supérieure des anses, est documenté par plusieurs exemplaires de la grotte. Il ne s'agit donc pas d'une forme spécifique, créée pour les besoins du sanctuaire ${ }^{38}$. Il est toutefois possible que la forme soit liée au culte de la grande déesse d'Amathonte, honorée au palais comme au sanctuaire du sommet de l'acropole, car aucun autre contexte amathousien n'en a livré. Plusieurs fragments d'anses trifides, dont un de fabrique Bichr.R., appartiennent à ce type (fig. 105).

37. Th. PetIT, «Le palais », dans P. AuperT (dir.), Guide d'Amathonte, SitMon XV (1996), p. 99-107. Il est d'ailleurs remarquable que les pithoi retrouvés lors des fouilles des magasins du palais offrent une grande variété de types.

38. Contrairement à ce que j'ai écrit dans Amathonte VI, p. 72-73. Le type est peut-être aussi connu parmi le matériel du palais de l'acropole : $B C H 114$ (1990), p. 996, fig. 12. 
J'avais placé cette forme, jusqu'alors inédite, dans la classe IV, en m'appuyant sur des critères morphologiques et stylistiques. Cependant, l'absence d'amphores de type A.1 dans le bothros et sa présence dans les deux grands dépôts de la fin de l'archaïsme, celui de la grotte et celui du rempart Nord, invitent peut-être à revoir cette attribution. Certes, le décor végétal qui orne souvent les cols trouve ses meilleurs parallèles parmi les productions d'autres régions des classes III et IV ${ }^{39}$, mais c'est un répertoire qui ne devient en vogue dans le royaume qu'au Chypro-Archaïque II, avec le «style d'Amathonte ». Certes, le décor figuré de l'" amphore aux taureaux » renvoie à des œuvres levantines du début du VIIe s. av. J.-C., mais la reprise archaïsante de modèles orientaux ou égyptiens plus anciens semble un trait caractéristique des ateliers amathousiens du VIe s. ${ }^{40}$. Par ailleurs, certains motifs décoratifs (fig. 104) et l'usage de la technique du «Bichrome amathousien » sont des traits caractéristiques du ChyproArchaïque II, même s'ils peuvent apparaître plus tôt dans les productions du royaume.

\section{A.2. Anses de la lèvre à l'épaule (fig. 106-108)}

A.2.1. Col cylindrique ou légèrement évasé, lèvre en bourrelet externe (fig. 106)

Tête de série : $\mathrm{d} / \mathrm{a}$.bor.3.

Le type n'est illustré que par un fragment d'amphore BoR, probablement résiduelle.

A.2.2. Col cylindrique ou légèrement évasé, lèvre étalée (fig. 107)

Tête de série : d/a.pw.15.

Le type est tout aussi rare dans le dépôt que le précédent, puisqu’on n’a pu lui rattacher qu'un fragment PW avec anse trifide ${ }^{41}$.

A.2.3. Col nettement évasé (fig. 108)

Tête de série : $\mathrm{d} / \mathrm{a}$.bichr.5.

B. AMPHORE A ANSES HORIZONTALES (fig. 109-138)

B.1. Col proportionnellement court par rapport à la hauteur totale du vase, lèvre en bourrelet externe (fig. 109110)

39. Comparer notamment le décor de d/a.wp.1 et 2 (fig. 99-100) avec celui des amphores à col haut WP et Bichr. IV, sans doute fabriquées dans la partie orientale de l'île (Salamine), comme l'amphore Hubbard du Musée de Chypre : SCE IV/2, pl. 23, 18. Pour ce style qu'elle nomme "fleuri ", voir également M. YoN, « Peintres, potiers et coroplathes à Salamine », CCEC 35 (2005), p. 35-54.

40. Voir le commentaire d'A. HERMARY, Amathonte $V$. Les figurines en terre cuite archaïques et classiques, les sculptures en pierre, ÉtChypr XV (2000), p. 79-80, cat. no 514. Pour l'étude du décor du vase aux taureaux : id., BCH 114 (1990), p. 187-206.

41. Ce type d'anse complexe, qui imite probablement des attaches de vases métalliques, est connu depuis le début de l’Âge du Fer : voir, par exemple, une amphore récemment découverte dans la nécropole de Palaepaphos-Plakes : BCH 124 (2000), p. 673, fig. 27.

BCH 133 (2009) 
B.1.1. Amphore de grande taille à col cylindrique, de fabrique hybride (fig. 109-110)

Têtes de série : $\mathrm{d} /$ a.bor.2, d/a.bichr.r.4.

Cette série, dont la diffusion paraît limitée aux régions d'Amathonte et de Kourion ${ }^{42}$, est caractérisée par une technique de décoration mixte ou hybride : le col porte, sur sa surface extérieure, un décor peint sur fond blanc ou réservé (fabriques WP ou Bichr.) et, sur sa surface intérieure, un engobe rouge ou noirci. Le corps du vase est décoré selon une technique de peinture sur engobe rouge, monochrome $(\mathrm{BoR})$ ou, plus fréquemment, bichrome (Bichr.R.), qui peut être également noircie (fabriques « Blackened»).

\section{B.2. Col cylindrique, lèvre en bourrelet externe (fig. 111-115)}

B.2.1. Col assez haut et étroit, corps globulaire (fig. 111-114)

Têtes de série : d/a.pw.17, d/a.pw.19, d/a.wp.4, d/a.wp.6, d/a.bichr.r.1.

L'attribution à ce type des amphores PW est loin d'être assurée. Le petit nombre d'anses verticales dans le dépôt et l'absence de traces d'arrachement d'anses sur le col invitent à leur restituer des anses horizontales, fixées sur l'épaule. Leurs autres caractéristiques toutefois, et en particulier leurs dimensions, les rapprochent des amphores pithoïdes de type A.1.

Ce type est commun en fabrique WP, et il entre dans la classe IV. Sa présence, assez nombreuse, dans des contextes plus récents montre qu'il continue d'être produit au cours du VIe s. av. J.-C. Les autres amphores portent généralement un décor géométrique (lignes et cercles concentriques), où s'immiscent parfois des motifs végétaux (fig. 113-114). Le décor figuré de l'amphore d/a.bichr.r.1 est exceptionnel ${ }^{43}$.

B.2.3. Col assez haut et étroit, moulures sur la lèvre et le col (fig. 115)

Tête de série : d/a.pw.20.

Comp. : Amathonte : Fr. Vandenabeele, BCH 109 (1985), p. 645, fig. 67 (PW IV).

Marion : SCE IV/2, fig. XLV, 18 (PW IV).

Ce type, qui n'est illustré que par deux individus PW du rempart Nord, n'avait pas été recensé parmi le matériel du sanctuaire. Il est relativement rare, mais on retrouve le détail du col à ressaut sur l'abondante série des amphores à anses de panier (type C.1). Comme ces dernières, les amphores à col étroit et lèvre moulurée doivent apparaître au ChyproArchaïque I et continuer d'être fabriquées au VIe s. av. J.-C. L'attribution à la classe IV, proposée par E. Gjerstad pour l'exemplaire, voisin, de Marion, semble donc raisonnable.

42. On complètera les références données dans Amathonte VI par l'article de L. AlPE, S. FOURRIER, "Une production originale d'Amathonte : les amphores à anses horizontales et les dinoi de style hybride ", CCEC 33 (2003), p. 149-167.

43. L'amphore est publiée en détail dans BCH 132 (2008), p 561. 
Le type s'inscrit probablement dans la catégorie des grandes amphores de fabrique PW, de dimensions parfois proches de celles des pithoi, que leurs variantes morphologiques font entrer dans divers types (A.1, A.2, B.2.1). Comme les pithoi, qui présentent eux aussi de nombreuses variantes, ces amphores de grandes dimensions paraissent, à Amathonte, spécifiques des assemblages palatiaux ${ }^{44}$.

\section{B.3. Amphorisque à col légèrement évasé45 (fig. 116-119)}

Têtes de série : d/a.pw.23, d/a.bichr.9, d/a.bichr.10, d/a.bichr.11, d/a.bichr.12, d/a.bichr.13, da.bichr.14, d/a.bichr.15, d/a.bichr.16, d/a.bichr.17, d/a.bichr.18, d/a.bichr.r.3, d/a. bs.3, d/a.bs.2.

Le type est caractéristique des assemblages céramiques d'Amathonte à la fin de l'époque archaïque. Les exemplaires Bichr. décorés dans le «style d'Amathonte » ont été publiés en détail dans un article précédent ${ }^{46}$. Les amphorisques Bichr.R. et Bichr.R. "Blackened" portent un décor répétitif, fait de frises géométriques sur le col et l'épaule, que rehausse une ligne de points blancs à la jonction du col et de la panse, et de croisillons ou de lignes ondulées blancs sur le panneau principal, qui court entre les deux anses. Sur certains exemples, le haut du col, à l'extérieur, n'est pas engobé et la peinture noire est appliquée directement sur la surface, selon la technique WP (fig. 118). Contrairement aux vases du "style d'Amathonte ", relativement rares, les amphorisques à croisillons ou lignes ondulés abondent dans le matériel des nécropoles du royaume à la fin du VIe s. av. J.-C.

\section{B.4. Col nettement évasé ${ }^{47}$ (fig. 120-135)}

B.4.1. Lèvre en bourrelet externe (fig. 120-132)

Têtes de série : d/a.pw.21, d/a.pw.22, d/a.wp.5, d/a.wp.7, d/a.wp.8, d/a.wp.9, d/a.bichr.7, d/a.bichr.8, d/a.bor.4, d/a.bichr.r.5, d/a.bichr.r.6, d/a.bs.9, d/a.bs.10.

Il s'agit là encore d'un type qui est très commun à Amathonte et sur le territoire du royaume dans les assemblages céramiques, en particulier funéraires, de la fin de l'archaïsme. À l'évasement du col correspond un corps souvent biconique, qui contribue à donner aux vases de cette série un profil très écrasé, caractéristique des productions amathousiennes

44. L'origine du matériel utilisé pour combler le silo de l'acropole, publié par Fr. VANDENABEELE (BCH 109 [1985], p. 629-655), est inconnue, mais la localisation du dépôt, en contrebas du palais, invite à y voir un dépôt palatial plutôt que domestique.

45. La cohérence de la série m'incite à fondre en un seul type B.3 les deux variantes que j’avais distinguées dans Amathonte VI, p. 77.

46. BCH 132 (2008), p. 000.

47. L'existence d'une variante avec lèvre repliée m'a conduite à distinguer deux sous-types à l'intérieur de cette catégorie, qui n'était jusqu'alors illustrée que par des exemplaires à lèvre en bourrelet externe : Amathonte VI, p. 78. Les parallèles proposés dans cette dernière publication concernent donc désormais le type B.4.1.

BCH 133 (2009) 
du Chypro-Archaïque II. Les amphores sont de dimensions variées et le dépôt du rempart Nord a livré quelques exemplaires d'assez grande taille.

B.4.2. Lèvre repliée (fig. 133-135)

Têtes de série: $\mathrm{d} / a . b i c h r . r .7, \mathrm{~d} / a . b s .7, \mathrm{~d} /$ a.bs.5.

Comp. : Marion : SCE IV/2, fig. XLVII, 1 (WP V) ; fig. L, 9 (Bichr. V).

Tremithousa : E. RAPTOU, RDAC 2001, p. 192, fig. 3, 22 (WP VI).

La variante à lèvre repliée n'est pas attestée parmi le matériel archaïque du sanctuaire et c'est un trait morphologique qui est, de manière générale, peu prisé des potiers amathousiens. De fait, la forme générale de ces amphores (notamment, outre la lèvre repliée, le col haut), la technique du décor (qui marie une technique Bichr. en noir et rouge, et non pas noir et blanc, sur une surface qui paraît déjà recouverte d'un engobe rouge) et la qualité de la pâte (beige, grise au cœur) orientent vers l'hypothèse d'une production non-amathousienne. Les cols hauts et larges, ornés de cercles concentriques et fermés d'une lèvre repliée, paraissent des traits propres aux ateliers du Nord-Ouest de Chypre et, en particulier de Paphos, où des amphores de ce type sont fabriquées dès le ChyproArchaïque I ${ }^{48}$. La découverte d'une amphore à col haut et lèvre repliée dans une tombe d'époque classique de Tremithousa, non loin de Paphos, confirme la permanence de la forme tout au long de la période archaïque.

La série du rempart Nord, en nombre réduit, est très cohérente, et dans la forme, et dans le schéma décoratif. Sur les quatre individus du dépôt, trois sont de fabrique Bichr.R. "Blackened", un de fabrique Bichr.R. Les amphores de Marion possèdent un col très évasé et un corps nettement biconique, traits tardifs qui paraissent moins marqués sur les exemplaires recueillis dans le dépôt du rempart Nord. Néanmoins, une attribution de l'ensemble de cette série à la classe V, caractéristique du Chypro-Archaïque II, est satisfaisante en l'état actuel de la documentation, même si le type est encore attesté dans des contextes plus récents ${ }^{49}$.

\section{B.5. Amphore miniature à col évasé, lèvre ronde, anses en ruban (fig. 136-138)}

Têtes de série : d/a.pw.24, d/a.bichr.19, d/a.bichr.20.

La forme, quoique rare, est typique des productions amathousiennes de la fin de l'époque archaïque. On remarquera la présence d'un exemplaire PW50.

48. Par exemple, V. KarageOrGHIS, BCH91 (1967), p. 226, fig. 12 (nécropole de Palaepaphos-Mavrommatis). On remarquera également que les vases BoR archaïques de Paphos possèdent une même pâte assez fine, grise au cœur.

49. La tombe de Tremithousa est datée du IVe s. av. J.-C. par son inventeur : E. RAPTOU, RDAC 2001, p. 187-212.

50. Ce type est surtout décliné en fabriques peintes, et notamment en Bichr. Mentionnons toutefois une amphore miniature PW de la Tombe 646 d'Amathonte : P. FlourENTZOS (supra, n. 9), p. 221, 52 (PW V).

BCH 133 (2009) 


\section{AMPHORE À ANSES DE PANIER ET VARIANTES (fig. 139-147)}

Il n'est pas question de reprendre ici en détail le dossier des amphores à anses de panier, qui représentent une catégorie importante non seulement à Chypre mais aussi dans l'ensemble de la Méditerranée orientale où ce type a été largement exporté dès l'époque archaïque ${ }^{51}$. On se contentera d'ajouter, aux subdivisions proposées dans Amathonte VI, un nouveau type, C.3, qui correspond à la forme évoluée de cette amphore de transport chypriote. Le type C.2, qui était illustré dans la grotte du sanctuaire par un exemplaire complet, n’a pas pu être identifié parmi les fragments du rempart Nord.

La plupart des exemplaires recueillis dans le dépôt du rempart Nord possèdent une pâte typiquement amathousienne, qui en prouve le caractère local. Ce type, rare au sanctuaire, est très commun dans le dépôt palatial, ainsi qu'au palais d'époque classique. Comme au palais, plusieurs fonds d'amphores à anses de panier du dépôt du rempart Nord portent des marques incisées avant cuisson ${ }^{52}$.

\section{C.1. Lèvre repliée, ressaut sur le col, corps de profil convexe ou biconvexe (fig. 139-141)}

Têtes de série : d/a.pw.7, d/a.pw.8, d/a.pw.9.

Les amphores du dépôt du rempart Nord possèdent des traits évolués, qui invitent à les placer dans la classe V. De fait, le col est plus large et plus court que celui des exemplaires du bothros, ce qui invite à leur restituer un corps biconvexe et des anses de panier qui dépassent largement de l'ouverture du col. Comme celles, plus anciennes, du bothros toutefois, les amphores du rempart Nord devaient être munies d'une base annulaire ${ }^{53}$.

\section{C.3. Col court, anses placées haut sur l'épaule, fond pointu (fig. 142-147)}

Têtes de série : d/a.pw.1, d/a.pw.2, d/a.pw.4, d/a.pw.6.

Ce type illustre la forme évoluée de l'amphore à anses de panier, au corps plus allongé, aux anses verticales et au fond pointu (fig. 146-147). C'est la forme caractéristique de l'époque classique, qui apparaît dès la fin du VI ${ }^{\mathrm{e}} \mathrm{s}$. av. J.-C. ${ }^{54}$. La lèvre des différents exemplaires présente des variantes de détail, qui ne paraissent pas avoir de signification chronologique. Certains fonds, en revanche, dont la surface a été raclée verticalement, sont caractéristiques de la classe VI55.

51. Voir les indications et les références bibliographiques données dans Amathonte VI, p. 78-79.

52. S. FOURRIER, BCH 128-129 (2004-2005), p. 92-95.

53. On complètera les exemplaires fragmentaires du dépôt selon le modèle de l'amphore de Marion, illustrée dans SCE IV/2, fig. LVII 22.

54. Voir SCE IV/2, fig. LVII 23 (amphore PW V de Marion).

55. Comparer fig. 146 et SCE IV/2, fig. LXIII 10 (amphore PW VI de Vouni).

BCH 133 (2009) 
D. AMPHORE À FOND EN BOUTON (fig. 148)

Tête de série : $\mathrm{d} / \mathrm{a} \cdot \mathrm{pw} .16$.

Il s'agit du type de l'amphore commerciale grecque, qui est bien attesté dans le dépôt par de nombreuses importations de l'Attique, de Corinthe et de la Grèce de l'Est. Quelques fragments, auxquels je n'ai pu rattacher aucun rebord, paraissent toutefois, en raison de leur pâte, appartenir à une amphore de production chypriote mais non locale. Le type est inclus dans la classification suédoise dans la classe $\mathrm{V}^{56}$.

\section{4. JARRES}

Les jarres constituent une catégorie peu nombreuse, mais représentée par une grande variété de types, au sein du dépôt du rempart Nord. J'ai donc ajouté un certain nombre de subdivisions à la typologie proposée dans Amathonte VI, certaines d'entre elles n'étant illustrées que par un exemplaire.

\begin{tabular}{l|r|r|r|r|r|r|r|r} 
Type & PW & WP & Bichr. & \multicolumn{1}{|l|}{ RS } & BS & BoR & $\begin{array}{c}\text { Bichr.R. et } \\
\text { "Blackened }\end{array}$ & Total \\
\hline \hline A.1 & 0 & 0 & 0 & 0 & 0 & 1 & 0 & 1 \\
\hline A.4 & 0 & 0 & 2 & 0 & 0 & 0 & 0 & 2 \\
\hline A.5 & 0 & 0 & 0 & 1 & 0 & 0 & 0 & 1 \\
\hline B.2 & 71 & 1 & 0 & 1 & 0 & 0 & 1 & 74 \\
\hline C.1 & 18 & 0 & 0 & 2 & 0 & 3 & 0 & 23 \\
\hline C.3 & 4 & 6 & 12 & 1 & 0 & 2 & 6 & 31 \\
\hline TOTAL & $\mathbf{9 3}$ & $\mathbf{7}$ & $\mathbf{1 4}$ & $\mathbf{5}$ & $\mathbf{0}$ & $\mathbf{6}$ & 7 & $\mathbf{1 3 2}$ \\
\hline \hline
\end{tabular}

Tableau 7. - Répartition des jarres par type et par fabrique.

A. À COl distingué de LA PANSE (fig. 149-152)

A.1. Col cylindrique court, lèvre ronde, anses horizontales (fig. 149)

Tête de série : $\mathrm{d} / \mathrm{d} .12$.

Le type n'est illustré que par un fragment de col. Cet unique exemplaire porte vraisemblablement un décor hybride, peint en noir sur surface réservée sur le haut du col (technique WP), en noir ou en noir et blanc sur engobe rouge pour le reste du vase (technique BoR ou Bichr.R.). Un parallèle exact est fourni par une jarre Bichr.R. I(IV) de la

56. SCE IV/2, fig. LVII 24-25 (amphores PW V de Marion).

BCH 133 (2009) 
Tombe 556 d'Amathonte-Kokkinogia 57. Comme les amphores de type B.1.1, cette nouvelle catégorie de vases hybrides représente une production spécifiquement amathousienne.

\section{A.4. Col cylindrique court, lèvre étalée (fig. 150-151)}

Têtes de série: $\mathrm{d} / \mathrm{d} .1, \mathrm{~d} /$ cr.bichr. $3^{58}$.

Comp. : Limassol-Aghia Zoni : BCH 86 (1962), p. 379, fig. 73, droite. Marion : SCE IV/2, fig. XXXI, 9 (Bichr. IV).

Les deux vases Bichr. que je place dans ce type ont en commun de posséder un col court et une lèvre étalée. Il n'est pas sûr qu'ils entretiennent d'autres liens morphologiques, ce que leur état de conservation ne permet pas de décider.

Le rapprochement avec la petite jarre de Limassol-Aghia Zoni n'est pertinent que pour $\mathrm{d} /$ cr.bichr.3. On remarquera que, comme cette dernière, l'exemplaire du rempart Nord porte un décor caractéristique du "style d'Amathonte ", ce qui conduit à le placer dans la classe Bichr. V. La pâte de l'autre jarre (fig. 150) montre qu'elle est importée d'une autre région de Chypre. Sa texture, ainsi que le traitement de surface du vase, invitent à le rapprocher des amphores de type B.4.2 (fig. 133-135), que j’ai proposé d'identifier comme paphiennes.

\section{A.5. Rebord évasé, lèvre biseautée (fig. 152)}

Tête de série : $\mathrm{d} / \mathrm{d} .2$.

Comp. : ?

Ce type de col est attesté, dès le début de l'Âge du Fer, sur des marmites ${ }^{59}$. Il paraît toutefois plus fréquent sur des jarres des classes $\mathrm{V}$ et, surtout, VI ${ }^{60}$, même si aucun des rapprochements proposés n'est exactement semblable à notre fragment.

57. BCH 113 (1989), p. 810, fig. 78. Le même schéma décoratif sur le col, réservé, apparaît sur une jarre Bichr.R. I(IV) de forme sensiblement différente : SCE IV/2, fig. XLI 1, qu'on peut rapprocher de fragments recueillis dans le silo, en contrebas du palais : Fr. VANDENABEELE, BCH 109 (1985), p. 652, fig. 116-117.

58. J'avais d'abord compris ce fragment comme appartenant à une cruche, d'où son no d'inventaire. Cependant, le rapprochement avec la petite jarre de Limassol-Aghia Zoni invite à revoir cette interprétation et à restituer deux anses verticales au vase, ce qui n'apparaît pas sur le dessin fig. 151.

59. V. Karageorghis, Palaepaphos-Skales. An Iron Age Cemetery in Cyprus (1983), p. 368. Pour des contextes de la fin du Chypro-Archaïque I : id. (1967, supra, n. 23), pl. CXXV 75.

60. SCE IV/2, fig. LII 4 (BoR III[V]) ; fig. LIX 5 (Bichr. VI) ; fig. LX 3 (BoR IV[VI]) et 9 (Bichr.R III[VI]) ; fig. LXII 1 (PW VI). On peut également rapprocher la forme générale de la jarre d'Amathonte, mais non le détail de sa lèvre, de la série des " cratères " des niveaux classiques de Kition-Bamboula: KB II, fig. 31, 264-265.

BCH 133 (2009) 


\section{B. À COL NON DiSTINGUÉ DE LA PANSE ET OUVERTURE TRÈS LARGE (fig. 153-155)}

\section{B.2. Lèvre en bourrelet externe (fig. 153-155)}

Têtes de série : d/b.pw.34, d/b.pw.37, d/d.3.

Le type est bien représenté en fabrique PW. Les vases sont souvent d'assez grandes dimensions et ils sont munis d'anses verticales. Leur morphologie les rapproche des marmites ${ }^{61}$, mais leur pâte, calcaire, et leur surface, qui n’a jamais été exposée au feu, montrent qu'ils avaient un autre usage.

C. À COL NON DISTINGUÉ DE LA PANSE ET OUVERTURE RÉDUITE PAR RAPPORT AU DIAMÈTRE DU CORPS (fig. 156-161)

C.1. Lèvre ronde ou épaissie, dans le prolongement de la paroi ou légèrement redressée (fig. 156-157)

Têtes de série : $\mathrm{d} / \mathrm{b} \cdot \mathrm{pw} .36, \mathrm{~d} / \mathrm{d} .4$.

Les jarres de type C.1 sont très fragmentaires. Elles reprennent une forme d'origine levantine, connue à Chypre dès le Chypro-Géométrique III ${ }^{62}$. Le choix de fabriques sans décor $(\mathrm{PW})$ ou à engobe rouge (RS et BoR) souligne cette filiation.

Le prototype est dépourvu d'anses. Lorsqu'elles sont suffisamment bien conservées, les jarres du dépôt possèdent des anses verticales, fixées haut sur l'épaule et très débordantes. Cela invite à leur prêter un profil plus biconique que globulaire, à l'image d'une jarre de Marion, caractéristique de la classe $\mathrm{V}^{63}$. Mais le meilleur parallèle est sans doute fourni par deux jarres découvertes à Aghia Phyla-Limnazousa, tout près de Limassol, et elles aussi datables du Chypro-Archaïque II : ces dernières, qui possèdent toutefois une lèvre repliée et non ronde, portent un décor hybride, caractéristique des ateliers amathousiens ${ }^{64}$.

\section{C.3. Courte lèvre étalée (fig. 158-161)}

Têtes de série : d/d.6, d/d.7, d/d.8, d/d.9.

Comp. : ?

61. En particulier des jarres $\mathrm{CW}$ de grandes dimensions, dont plusieurs exemplaires ont été recueillis dans le dépôt du rempart Nord : $B C H 131$ (2007), p. 74-75 et p. 90-91, fig. 13-15.

62. Elle est répertoriée dans la typologie suédoise dès la classe III : SCE IV/2, fig. XXVII 2 (jarre RS I[III] d'Amathonte). Un exemple importé, muni d'un pied complexe en anneaux, provient de la nécropole d'Amathonte : P. MAYNOR BIKAI, dans La nécropole d'Amathonte, tombes 113-367, II. Céramiques non chypriotes, Et Chypr VIII (1987), pl. VII 17 ; un autre, de fabrique RS locale (?) du "pseudo-tophet " : BCH 117 (1993), p. 726, fig. 19.

63. SCE IV/2, fig. LIII 10 (Bichr.R. II[V]). Cette dernière possède cependant une lèvre repliée et non ronde.

64. BCH 90 (1966), p. 332, fig. 81. Voir également le commentaire de L. ALPE, S. FouRRIER (supra, n. 42), p. $160-161$. 
Cette dernière variante complète le groupe des jarres de type $\mathrm{C}$ répertoriées dans Amathonte $\mathrm{VI}{ }^{65}$. Elle est documentée par une petite série de vases décorés, surtout de fabrique Bichr., et même Polychrome, puisque des rehauts de peinture blanche, qui complètent le noir et le rouge, ne sont pas rares. La série est homogène, tant dans son format que dans son décor qui emprunte ses motifs principaux, géométriques et floraux, au «style d'Amathonte ».

Le type n'est pas recensé dans la typologie suédoise et je ne lui ai trouvé aucun parallèle parmi les découvertes publiées des nécropoles d'Amathonte et de sa région. La forme de la lèvre et de la vasque rapproche nos exemplaires amathousiens de certaines jarres de fabrique Bichr. IV, qui évoluent dans la classe suivante avec un corps moins globulaire ${ }^{66}$. Mais il est improbable que les jarres d'Amathonte soient munies d'un piédestal, et leur décor renvoie aux productions locales du Chypro-Archaïque II. Les traces d'arrachement d'une anse, visibles sur un seul des fragments recueillis, montrent que les jarres possédaient des anses verticales, dont l'attache supérieure était fixée juste sous le rebord.

\section{5. COUVERCLES}

Les couvercles recueillis dans le dépôt du rempart Nord n'apportent aucune nouveauté par rapport aux types répertoriés parmi le matériel du sanctuaire ${ }^{67}$. Ils sont, en outre, peu nombreux et ils possèdent tous un profil convexe (type A), sauf, peut-être, un fragment RS peu identifiable.

\begin{tabular}{l|r|r|r|r|r|r|r|r} 
Type & PW & WP & Bichr. & \multicolumn{1}{|c|}{ RS } & BS & BoR & $\begin{array}{c}\text { Bichr.R. et } \\
\text { ilackened }\end{array}$ & Total \\
\hline \hline A.1.1 & 5 & 7 & 12 & 4 & 0 & 1 & 0 & 29 \\
\hline A.2 & 0 & 0 & 2 & 0 & 0 & 0 & 1 & 3 \\
\hline B & 0 & 0 & 0 & $1 ?$ & 0 & 0 & 0 & $1 ?$ \\
\hline TOTAL & $\mathbf{5}$ & 7 & $\mathbf{1 4}$ & $\mathbf{5}$ & $\mathbf{0}$ & $\mathbf{1}$ & $\mathbf{1}$ & $\mathbf{3 3}$ \\
\hline \hline
\end{tabular}

Tableau 8. - Répartition des couvercles par type et par fabrique.

A. PROFIl CONVEXE (fig. 162-167)

A.1. Profil de courbe convexe plus ou moins prononcée, lèvre étalée (fig. 162-165)

Têtes de série: $\mathrm{d} / \mathrm{c} .1, \mathrm{~d} / \mathrm{c} .2, \mathrm{~d} / \mathrm{c} .3, \mathrm{~d} / \mathrm{c} .4$.

65. Amathonte VI, p. 81.

66. SCE IV/2, fig. XXXI 14 (jarre Bichr. IV du Musée de Chypre) ; fig. LII 5 (jarre BoR III[V] d'Amathonte).

67. Amathonte VI, p. 83-84.

BCH 133 (2009) 
Comme attendu, tous les couvercles du rempart Nord entrent dans le type A.1.2, à longue lèvre horizontale, qui représente une forme évoluée du type A.1.1, qui n'est attesté, au sanctuaire, que parmi le matériel du bothros. Certains profils sont très écrasés (fig. 164), ce qui confirme une datation à la fin de l'époque archaïque.

\section{A.2. Profil globulaire (fig. 166-167)}

Têtes de série $: \mathrm{d} / \mathrm{c} .6, \mathrm{~d} / \mathrm{c} .7$.

Les couvercles de ce type se distinguent des précédents par une courbe continue de la paroi, que ne vient pas casser l'orientation de la lèvre. On remarquera le décor caractéristique du «style d'Amathonte » que porte l'exemplaire Bichr. (fig. 167).

\section{LES FABRIQUES}

\begin{tabular}{l|r|r|r|r|r|r} 
& \multicolumn{3}{|c|}{ Rempart Nord } & \multicolumn{3}{c}{ Grotte } \\
\hline \hline Fabriques & NR & NMI & \multicolumn{1}{c|}{$\%$} & NR & NMI & $\%$ \\
\hline PW & 31537 & 2474 & $43,2 \%$ & 16856 & 1471 & $40,7 \%$ \\
\hline WP & 7797 & 821 & $14,3 \%$ & 5158 & 579 & $16 \%$ \\
\hline Bichr. & 3387 & 554 & $9,7 \%$ & 3807 & 573 & $15,9 \%$ \\
\hline RS & 2128 & 149 & $2,6 \%$ & 1369 & 109 & $3 \%$ \\
\hline BoR & 1007 & 179 & $3,1 \%$ & 625 & 130 & $3,6 \%$ \\
\hline Bichr.R., BS et fabriques & 5898 & 735 & $12,8 \%$ & 710 & 126 & $3,5 \%$ \\
\hline «lackened» & 189 & 146 & $2,5 \%$ & 1178 & 509 & $14,1 \%$ \\
\hline Paroi fine & 2845 & 131 & $2,3 \%$ & 1715 & 60 & $1,7 \%$ \\
\hline Jarres-torpilles & 7571 & 542 & $9,5 \%$ & 547 & 55 & $1,5 \%$ \\
\hline Culinaires & $\mathbf{6 2 3 5 9}$ & $\mathbf{5 7 3 1}$ & $\mathbf{1 0 0} \%$ & $\mathbf{3 1 9 6 5}$ & $\mathbf{3 6 1 2}$ & $100 \%$ \\
\hline TOTAL & & & & &
\end{tabular}

Tableau 9. - Répartition des différentes fabriques. Comparaison entre les dépôts du rempart Nord et de la grotte du sanctuaire ${ }^{68}$.

68. Afin de permettre la comparaison avec le tableau publié dans Amathonte VI, p. 117, les jarres-torpilles ont été intégrées dans le dénombrement, alors qu'il ne s'agit pas d'une production chypriote, et un champ spécifique a été créé pour les bols à paroi fine, qui, selon leur mode de décoration, entrent dans diverses fabriques. En revanche, les fabriques BS, Bichr.R. et dérivées "Blackened " n’ont pas été distinguées dans la mesure où l'identification est, à de rares exceptions près, très difficile à partir de fragments.

BCH 133 (2009) 


\section{1. PW}

\section{1. 1. Formes ouvertes}

Parmi les bols de type A.1 domine la variante à lèvre biseautée.

Type A.1.1

d/b.pw.10 (fig. 1). - NR = 1. Diam. 20,4 cm. Pâte rose, nombreuses inclusions noires et blanches.

Type A.1.3

d/b.pw.17 (fig. 7). - NR = 2. Diam. 28,8 cm. Pâte rose, petites inclusions noires et blanches. Petites anses horizontales en ruban.

Les petits bols sans anse, à lèvre légèrement rentrante (type A.2.1), sont particulièrement fréquents. Ils sont parfois déclinés en miniatures.

d/b.pw.22 (fig. 17). $-\mathrm{NR}=8$. Diam. $11 \mathrm{~cm}$. Pâte beige, petites inclusions noires.

d/b.pw.26 (fig. 18). - NR = 1. Diam. 5,1 cm. Pâte beige, petites inclusions noires et blanches.

Les quelques individus de type A.2.5 entrent tous dans la variante à lèvre à ressaut interne.

d/b.pw.4 (fig. 21). — NR = 1. Diam. $12 \mathrm{~cm}$. Pâte rose, petites inclusions noires et blanches.

Les bols peu profonds à panse carénée sont en nombre réduit.

d/b.pw.27 (fig. 26). — NR = 1. Diam. 7 cm. Pâte beige, inclusions noires et blanches.

Les bols à parois évasées rectilignes sont également de petit format. Ils possèdent une lèvre ronde (type A.4.1) ou étalée (type A.4.2). La dernière forme est la plus commune. Malgré des variations de détail, en particulier dans la forme de la lèvre, plus ou moins horizontale, la série est très homogène : de dimensions réduites, les bols sont munis d'un fond plat saillant, ils possèdent des parois épaisses et très inclinées, et ils sont systématiquement dépourvus d'anses, autre indice en faveur d'une origine levantine du type.

d/b.pw.14 (fig. 29). $-\mathrm{NR}=1$. Diam. 11,6 cm. Pâte beige, petites inclusions noires.

d/b.pw.19 (fig. 30). $-\mathrm{NR}=4$. Diam. 10,6 cm. Pâte rose, nombreuses inclusions noires.

d/b.pw.21 (fig. 31). $-\mathrm{NR}=2$. Diam. $9 \mathrm{~cm}$. Pâte beige, nombreuses petites inclusions noires.

Le bol profond de type B.2.2 est isolé.

d/b.pw.18 (fig. 42). - NR = 2. Diam. $17 \mathrm{~cm}$. Pâte beige, inclusions noires et blanches.

Les bols à lèvre (type B.6) sont également rares dans cette fabrique.

d/b.pw.1 (fig. 49). - NR = 1. Diam. $11 \mathrm{~cm}$. Pâte rose, petites inclusions noires. Surface extérieure beige

Les bols à paroi fine, très nombreux, forment une série homogène, à vasque profonde et profil convexe (type C.1.1), de module constant. Les trois fragments de lèvre qui documentent le type C.1.3 sont trop réduits pour permettre de restituer la forme.

BCH 133 (2009) 
d/b.pw.6 (fig. 55). - NR = 8. Diam. 11,2 cm. Pâte beige à rose, petites inclusions noires. Surface non lissée.

d/b.pw.7 (fig. 56). — NR = 2. Diam. 11,8 cm. Même pâte et même aspect de surface.

Parmi les formes ouvertes PW, signalons enfin une série de cuvettes, de formes variées.

Type D.1

d/b/pw.15 (fig. 59). $-\mathrm{NR}=1$. Diam. ? Pâte beige, nombreuses inclusions noires.

Type D.2

d/b.pw.16 (fig. 60). - NR = 1. Diam. 39,4 cm. Pâte beige à verdâtre, nombreuses inclusions noires. Sur la face externe, deux larges incisions avant cuisson.

Les «bols perses » (type D.3) sont mieux attestés.

Type D.3.1

d/b.pw.27 (fig. 61). - NR = 4. Diam. 21,4 cm. Pâte rose, inclusions noires et blanches. Surface verdâtre.

d/b.pw.28 (fig. 62). - NR = 2. Diam. $22 \mathrm{~cm}$. Pâte beige, petites inclusions noires et blanches. Surface verdâtre.

Type D.3.2

d/b.pw.32 (fig. 63). - NR = 8. Diam. 28,4 cm. Pâte rose, inclusions noires et blanches. Surface verdâtre.

d/b.pw.33 (fig. 64). - NR = 2. Diam. $35 \mathrm{~cm}$. Pâte beige à verdâtre, nombreuses inclusions noires. Surface verte.

Type D.3.3

d/b.pw.31 (fig. 65). - NR = 11. Diam. max. 27,2 cm. Pâte rose, beige au cœur, inclusions noires et blanches.

\section{1. 2. Cruches}

Les cruches à ouverture ronde sont les plus nombreuses dans cette fabrique.

Type A.1.1

d/cr.pw.1 (fig. 66). $-\mathrm{NR}=1$. Haut. 12,5 cm. Pâte beige, petites inclusions noires et blanches. Surface beige.

Type A.1.2

AM 2624 (fig. 72). - NR = 1. Haut. 9,3 cm (sans l'anse débordante). Pâte beige, nombreuses petites inclusions noires.

AM 2628 (fig. 73). - NR = 1. Haut. 7,3 cm (sans l'anse débordante). Pâte brune, inclusions noires et blanches. La surface du vase est couverte de concrétions calcaires.

BCH 133 (2009) 
Type A.3

d/cr.pw.5 (fig. 75). $-\mathrm{NR}=2$. Haut. cons. $2 \mathrm{~cm}$. Pâte beige, petites inclusions noires.

Le type A.4, absent du sanctuaire, est bien attesté dans le dépôt du rempart Nord, comme dans les tombes amathousiennes de la fin de l'époque archaïque. On remarquera, sur l'exemple illustré, la carène de l'épaule et le décor de lignes parallèles incisées, autant de traits tardifs qui confortent une attribution à la classe VI, caractéristique du ChyproClassique I.

d/cr.pw.4 (fig. 79). - NR = 5. Haut. cons. 11,4 cm. Pâte rose, inclusions noires et blanches, surface beige.

Les cruches à ouverture pincée possèdent des traits morphologiques tardifs, qui invitent à les placer dans la classe V, parfois VI. Le fragment d/cr.pw.8 (fig. 82) présente une pâte qui n'est pas amathousienne, mais qui renvoie à des productions de Salamine ou de la région d'Idalion. Le décor en pastille rapportée sur le haut de l'anse en est un autre détail caractéristique ${ }^{69}$.

d/cr.pw.8 (fig. 82). $-\mathrm{NR}=2$. Haut. cons. $5,8 \mathrm{~cm}$. Pâte beige, fine, surface beige lissée.

d/cr.pw.9 (fig. 83). $-\mathrm{NR}=1$. Haut. 4,3 cm. Pâte beige à orangé, nombreuses inclusions noires. Surface beige.

Le type B.1.2 est documenté par un assez grand nombre d'exemplaires, tous très mal conservés.

d/cr.pw.11 (fig. 90). - NR = 4. Haut. cons. 2,9 cm (sans l'anse). Pâte beige à rose. Petites inclusions noires.

Mentionnons enfin un fragment de cruche à bec, qu'il n'a pas été possible d'identifier plus précisément.

d/cr.pw.7 (fig. 96). $-\mathrm{NR}=1$. Haut. cons. $9 \mathrm{~cm}$. Pâte beige orangé, grosses inclusions noires et blanches. Surface extérieure beige.

\section{1. 3. Amphores}

Les amphores PW à anses verticales sont rares. Trois fragments de rebords illustrent le type A.1, qui était jusqu'alors uniquement connu grâce à des amphores de fabriques décorées recueillies dans la grotte du sanctuaire.

d/a.pw.14 (fig. 97). - NR = 9. Haut. cons. $13,1 \mathrm{~cm}$; diam. $38 \mathrm{~cm}$. Pâte beige, inclusions noires et blanches. Surface beige.

d/a.pw.18 (fig. 98). $-\mathrm{NR}=1$. Haut. cons. $16 \mathrm{~cm}$; diam. $58 \mathrm{~cm}$. Pâte beige, grosses inclusions noires et blanches. Traces d'arrachement d'une anse sur le col.

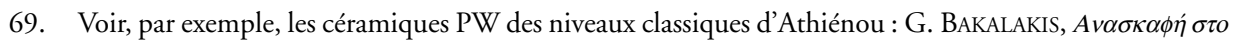

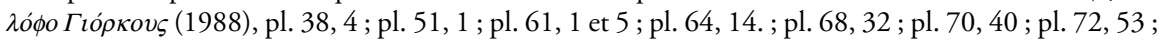
pl. 73, 6 .

BCH 133 (2009) 
Le fragment d'amphore de type A.2.2 se rapproche de la série de production précédente en raison de ses dimensions et du caractère composite de l'anse.

d/a/pw.15 (fig. 107). - NR = 3. Haut. cons. $23 \mathrm{~cm}$. Pâte beige, petites inclusions noires et blanches. Surface beige. Le boudin central de l'anse trifide est torsadé.

Il n'est pas assuré que les amphores classées dans le type B.2.1 possèdent des anses horizontales plutôt que verticales. Mais aucun fragment n'a permis d'identifier de départ d'anse sur le col et les fragments d'anses verticales sont, de manière générale, rares dans le dépôt du rempart Nord. Les nombreux exemplaires recensés sont proches, par leur morphologie générale, des amphores pithoïdes de type A.1. Seuls les deux vases illustrés possèdent des boutons décoratifs en pastilles de pâte rapportées.

d/a.pw.17 (fig. 111). $-\mathrm{NR}=1$. Haut. cons. 19,6 cm; diam. 35,3 cm. Pâte beige foncé, inclusions noires et blanches. Un bouton décoratif en pastille de pâte rapportée sur le col.

d/a.pw.19 (fig. 112). - NR = 2. Haut. cons. $17,8 \mathrm{~cm}$; diam. $50,5 \mathrm{~cm}$. Pâte beige foncé, inclusions noires et blanches. Surface verdâtre. Trois boutons décoratifs en pastilles rapportées sur le col.

Les amphores de type B.2.3 sont proches, bien que de dimensions légèrement plus réduites, du type précédent.

d/a.pw.20 (fig. 115). - NR = 3. Haut. cons. $16,8 \mathrm{~cm}$; diam. $24 \mathrm{~cm}$. Pâte beige, petites inclusions noires et blanches, surface verdâtre.

Une trentaine d'amphorisques PW documentent un type qui est surtout décliné en fabriques peintes.

d/a.pw.23 (fig. 116). $-\mathrm{NR}=6$. Haut. cons. $8,8 \mathrm{~cm}$, diam. $8,7 \mathrm{~cm}$. Pâte beige à rose, inclusions noires et blanches. Surface beige.

Le type d'amphores PW le plus commun se caractérise par un col nettement évasé et une lèvre en bourrelet externe (type B.4.1).

d/a.pw.21 (fig. 120). $-\mathrm{NR}=7$. Haut. cons. 12,2 cm ; diam. 19,6 cm. Pâte marron, inclusions noires et blanches. Surface beige.

d/a.pw.22 (fig. 121). $-\mathrm{NR}=6$. Haut. cons. $10,8 \mathrm{~cm}$; diam. 17,4 cm. Pâte beige, inclusions noires et blanches.

Dernier type à anses horizontales, les amphores miniatures ne sont documentées dans cette fabrique que par un fragment de panse et une anse en ruban, auxquels il a été impossible de rattacher un rebord.

d/a.pw.24 (fig. 136). - NR = 1. Haut. cons. $6 \mathrm{~cm}$. Pâte beige, petites inclusions noires et blanches.

Les amphores à anses de panier représentent une série importante et variée au sein du dépôt. 


\section{Type C. 1}

d/a.pw.7 (fig. 139). $-\mathrm{NR}=6$. Haut. cons. $8 \mathrm{~cm}$; diam. 10,9 cm. Pâte beige à rose, inclusions noires et blanches. Surface beige.

d/a.pw.8 (fig. 140). - NR = 2. Haut. cons. $7 \mathrm{~cm}$; diam. 12,8 cm. Pâte beige, petites inclusions noires et blanches. Surface beige.

d/a.pw.9 (fig. 141). - NR = 3. Haut. cons. $5,8 \mathrm{~cm}$; diam. $13 \mathrm{~cm}$. Pâte verdâtre, inclusions noires et blanches. Surface verdâtre.

Type C.3 :

d/a.pw.1 (fig. 142). - NR = 7. Haut. cons. $11,9 \mathrm{~cm}$; diam. 14,9 cm. Pâte rose orangé, nombreuses inclusions noires et blanches. Surface beige.

d/a.pw.2 (fig. 143). $-\mathrm{NR}=5$. Haut. cons. $5,8 \mathrm{~cm}$; diam. 14,1 cm. Pâte rose, nombreuses inclusions noires et blanches.

d/a.pw.4 (fig. 144). $-\mathrm{NR}=4$. Haut. cons. 4,6 cm ; diam. 12,8 cm. Pâte verte, grosses inclusions noires et blanches.

d/a.pw.6 (fig. 145). $-\mathrm{NR}=2$. Haut. cons. $4,7 \mathrm{~cm}$; diam. 12,2 cm. Pâte verdâtre, inclusions noires et blanches.

Plusieurs fragments appartiennent enfin à une amphore commerciale de type grec mais de production vraisemblablement chypriote (type D) :

d/a.pw.16 (fig. 148). $-\mathrm{NR}=4$. Haut. restituée (sans le rebord) 43,2 cm. Pâte beige, grosses et nombreuses inclusions noires.

\section{1. 4. Jarres}

Le type B.2 est documenté par un grand nombre d'exemplaires. La première jarre illustrée possède une pâte non amathousienne, qui invite à y reconnaître une importation de l'Est de l'île (Kition ?), mais je ne lui ai pas trouvé de parallèle.

d/b.pw.34 (fig. 153). - NR = 1. Diam. 16,9 cm. Pâte beige, fine et poudreuse, petites inclusions noires. Série d'incisions avant cuisson sur la panse.

d/b.pw.37 (fig. 154). - NR = 4. Diam. 25,4 cm. Pâte beige à rose, petites inclusions noires et blanches. Surface beige.

\section{Type C.1}

d/b.pw.36 (fig. 156). - NR = 1. Diam. $12 \mathrm{~cm}$. Pâte rose, nombreuses petites inclusions noires et blanches.

\section{2. WP}

\section{2. 1. Formes ouvertes}

Comme en fabrique PW, la variante à lèvre biseautée est largement majoritaire parmi les bols de type A.1.

BCH 133 (2009) 
Type A.1.2

d/b.wp.4 (fig. 4). - NR = 1. Diam. $23,2 \mathrm{~cm}$. Pâte verdâtre, très poreuse. Inclusions noires et blanches.

Type A.1.3

d/b.wp.5 (fig. 8). - NR = 4. Diam. 29,2 cm. Pâte beige, verdâtre par endroit, inclusions noires et blanches.

Les bols de type A.2 et A.3 sont rares dans cette fabrique.

Type A.2.1

d/b.wp.7 (fig. 19). — NR = 1. Diam. 17,7 cm. Pâte rose, inclusions noires et blanches.

Type A.3.2

d/b.wp.13 (fig. 27). — NR = 1. Diam. 13,8 cm. Pâte beige, petites inclusions noires et blanches.

Le type à parois rectilignes évasées et lèvre étalée (A.4.2) est mieux représenté, mais uniquement par des exemplaires fragmentaires.

d/b.wp.10 (fig. 32). — NR = 1. Diam. 26,4 cm. Pâte beige, petites inclusions noires et blanches.

Un fragment de lèvre pourrait illustrer le type profond B.2.2, mais il est de dimensions trop réduites pour que l'identification soit assurée. De même, il est possible qu'un certain nombre de bols, classés dans le type à panse carénée (B.3.2) soient en réalité de fabrique Bichr., ce que le mauvais état de conservation des surfaces ne permet pas de reconnaître sûrement.

d/b.wp.8 (fig. 44). - NR = 1. Diam. 21,4 cm. Pâte beige, inclusions noires et blanches.

Les petits bols sans anse à corps de courbe complexe (type B.4.2) sont peu nombreux, mais ils illustrent les tendances miniaturistes et décoratives des productions amathousiennes du Chypro-Archaïque II.

d/b.wp.15 (fig. 46). - NR = 6. Diam. 11,2 cm. Pâte beige, petites inclusions noires. Engobe blanc sur la surface extérieure.

\section{2. 2. Cruches}

Seul un exemplaire documente l'existence de cruches à ouverture ronde de fabrique WP. Le type est isolé, mais le mode d'attache de l'anse est assuré par les traces d'arrachement.

d/cr.wp.1 (fig. 74). — NR = 1. Haut. cons. $3,8 \mathrm{~cm}$. Pâte rose, inclusions noires et blanches. Pas d'engobe.

Les cruches à ouverture pincée sont plus nombreuses. 
Type B.1.1

d/cr.wp.2 (fig. 84). $-\mathrm{NR}=3$. Haut. cons. 7,7 cm (sans l'anse). Pâte blanchâtre, nombreuses petites inclusions noires.

Type B.1.2

d/cr.wp.5 (fig. 91). — NR = 7. Haut. cons. 10,3 cm (sans l'anse). Pâte beige, nombreuses inclusions noires.

La cruche de type B.2.1, caractéristique des assemblages du Chypro-Archaïque I, est résiduelle dans le dépôt du rempart Nord.

d/cr.wp.3 (fig. 93). $-\mathrm{NR}=1$. Haut. cons. 6,4 cm. Pâte beige, petites inclusions noires et blanches.

\section{2. 3. Amphores}

Les amphores à anses verticales sont beaucoup moins nombreuses que celles à anses horizontales. Seul un individu (non illustré) documente le type A.2.1. Tous les autres fragments entrent dans le type A.1.

d/a.wp.1 (fig. 99). - NR = 1. Haut. cons. $16,7 \mathrm{~cm}$; diam. $48 \mathrm{~cm}$. Pâte beige, friable, nombreuses inclusions noires et blanches. La surface est très abîmée.

d/a.wp.2 (fig. 100). $-\mathrm{NR}=1$. Haut. cons. $20 \mathrm{~cm}$; diam. $45 \mathrm{~cm}$. Pâte beige à verdâtre, friable. Inclusions noires et blanches. Engobe beige. Traces d'arrachement de l'anse sur le col.

Les amphores à anses horizontales et lèvre en bourrelet externe (B.2.1) sont nombreuses.

d/a.wp.4 (fig. 113). - NR = 7. Haut. cons. $17,4 \mathrm{~cm}$; diam. $31,2 \mathrm{~cm}$. Pâte beige à verdâtre, friable. Petites inclusions noires et blanches.

d/a.wp.6 (fig. 114). - NR = 13. Haut. cons. $18,6 \mathrm{~cm}$; diam. $30,4 \mathrm{~cm}$. Pâte beige, inclusions noires et blanches.

Les fragments d'amphorisques WP (type B.3) sont trop mal conservés pour être illustrés.

Les amphores à col évasé (type B.4.1), caractéristiques des productions amathousiennes du Chypro-Archaïque II, constituent une belle série, avec des exemplaires de différents modules, à décor géométrique.

d/a.wp. 5 (fig. 122). - NR = 12. Haut. cons. $19 \mathrm{~cm}$; diam. $34 \mathrm{~cm}$. Pâte verte, friable, nombreuses petites inclusions noires et blanches.

d/a.wp.7 (fig. 123). - NR = 136. Haut. cons. $36,7 \mathrm{~cm}$; diam. $22,5 \mathrm{~cm}$. Pâte verte à rose selon les zones, inclusions noires et blanches. Engobe blanc. Coup de feu sur la panse.

d/a.wp. 8 (fig. 124). - NR = 37. Haut. cons. $21 \mathrm{~cm}$; diam. 17,6 cm. Pâte beige à verdâtre, inclusions noires et blanches. Surface beige.

BCH 133 (2009) 
d/a.wp.9 (fig. 125). - NR = 1. Haut. cons. $7 \mathrm{~cm}$; diam. 18,2 cm. Pâte beige, inclusions noires et blanches.

\section{3. BICHR.}

\section{3. 1. Formes ouvertes}

Parmi les bols de type A.1, peu profonds et à épaule marquée, seule la variante à lèvre nettement biseautée est attestée en fabrique Bichr. Les motifs du décor sont répétitifs : outre les traditionnelles frises de lignes parallèles qui courent sur l'intérieur de la vasque et les cercles concentriques qui ornent le fond, la zone qui va de la lèvre à l'épaule porte, à l'extérieur, des séries de croisillons ou une large ligne ondulée, peints en rouge. Ces mêmes motifs sont déclinés, sur un bien plus grand nombre d'exemplaires et en peinture blanche, sur des bols de fabrique Bichr.R. et Bichr.R. «Blackened».

Type A.1.3

d/b.bichr.3 (fig. 9). $-\mathrm{NR}=4$. Diam. 14,4 cm. Pâte rose, inclusions noires et blanches.

d/b.bichr.5 (fig. 10). - NR = 3. Diam. 18,8 cm. Pâte rose, inclusions noires et blanches. Engobe blanchâtre, conservé seulement sur la paroi extérieure, de la lèvre à l'épaule.

Les quelques bols de type A.2.5 portent également un riche décor, caractéristique des productions amathousiennes de la fin de l'archaïsme, tant en raison de sa surcharge qu'à cause de l'emploi de rehauts de peinture blanche (il serait plus juste en l'occurrence de parler de fabrique Polychrome plutôt que Bichrome).

d/b.bichr.8 (fig. 22). - NR = 5. Diam. 11,5 cm. Pâte beige, inclusions noires et blanches. Surface lissée à l'extérieur ; engobe blanc sur la face interne. Le décor est peint en noir et rouge, rehauts de points blancs sur le cercle qui orne le fond.

Parmi les bols de type A.3.2, les formats réduits sont nombreux.

d/b.bichr.23 (fig. 28). - NR = 1. Diam. 6,4 cm. Pâte beige, petites inclusions noires. Le décor que supportait le fond est complètement effacé.

En revanche, les bols à parois rectilignes évasées et lèvre étalée (type A.4.2) sont de relativement grandes dimensions et ils portent un décor très coloré où la peinture rouge sert souvent de support à des motifs géométriques peints en noir, à l'imitation de la fabrique BoR (fig. 33).

d/b.bichr.12 (fig. 33a-b). - NR = 7. Diam. 22,4 cm. Pâte beige, très friable. Petites inclusions noires.

d/b.bichr.14 (fig. 34). - NR = 3. Diam. $21 \mathrm{~cm}$. Pâte beige, inclusions noires et blanches. Épais engobe blanc sur la surface interne.

On retrouve les tendances miniaturistes de la production amathousienne de la fin de l'archaïsme dans la série de petits bols de type B.2.1, qui portent un joli décor coloré, 
géométrique et floral. On remarquera que certains motifs (frises de chevrons, de feuilles ou de perles, fig. 38) sont typiques du «style d'Amathonte».

d/b.bichr.17 (fig. 36). - NR = 3. Diam. 13,6 cm. Pâte beige, petites inclusions noires. Engobe blanc sur la surface externe, de la lèvre à la large bande rouge qui marque l'épaule.

d/b.bichr.19 (fig. 37). - NR = 1. Diam. $13 \mathrm{~cm}$. Pâte beige, petites inclusions noires. La surface est très abîmée, rendant impossible la lecture du décor intérieur.

Le type B.2.2 est marginal parmi les bols Bichr.

d/b.bichr.20 (fig. 43). - NR = 1. Diam. 19,6 cm. Pâte beige à verdâtre, inclusions noires et blanches. La peinture rouge a viré au gris au cours de la cuisson (technique du "Bichrome amathousien »).

Type B.3.2

d/b.bichr.10 (fig. 45). - NR = 2. Diam. $20 \mathrm{~cm}$. Pâte beige, inclusions noires et blanches. Engobe beige lissé. Rehauts de peinture blanche (technique Polychrome).

Les bols miniatures (type B.5.2) sont une forme rare mais typique des productions amathousiennes de la fin de l'archaïsme.

d/b.bichr.25 (fig. 48). - NR = 1. Diam. 6,5 cm. Pâte rose, serrée, petites inclusions noires.

Isolé dans son type (B.6.1), le bol d/b.bichr.26 présente un même décor caractéristique des productions amathousiennes de la fin du Chypro-Archaïque II.

d/b.bichr.26 (fig. 50). - NR = 1. Diam. $13 \mathrm{~cm}$. Pâte beige, petites inclusions noires. Épais engobe blanc.

Les quelques bols à paroi fine, décorés d'une bande rouge sur le haut de la vasque, sont probablement des éléments résiduels dans le dépôt du rempart Nord.

Type C.1.3

d/b.bichr.27 (fig. 57). - NR = 3. Diam. 12,9 cm. Pâte rose, inclusions noires et blanches. Peinture rouge très écaillée sur la face interne.

Type C.2.2

d/b.bichr.28 (fig. 58). — NR = 3. Diam. 9,1 cm. Même pâte et même aspect de surface.

\section{3. 2. Cruches}

Les cruches à ouverture ronde sont rares, et elles documentent des types isolés. Leurs traits, morphologiques et décoratifs, sont caractéristiques des productions amathousiennes de la fin de l'époque archaïque, en particulier la lèvre pendante et l'usage de la seule peinture rouge, sans noir associé. 


\section{Type A.3}

d/cr.bichr.4 (fig. 76). - NR = 1. Haut. cons. 1,8 cm. Pâte rose, petites inclusions noires. Pas d'engobe visible. Le décor conservé est seulement peint en rouge.

Parmi la série des cruches à ouverture pincée, on remarque plusieurs exemples de type B.1.1 à décor d'œil sur le col.

d/cr.bichr.5 (fig. 85) ${ }^{70} .-N R=17$. Haut. 12,4 cm. Pâte rose, inclusions noires et blanches. Engobe blanchâtre.

d/cr.bichr.7 (fig. 86). $-\mathrm{NR}=1$. Haut. cons. $5,2 \mathrm{~cm}$. Pâte beige, petites inclusions noires. Engobe blanchâtre.

d/cr.bichr.9 (fig. 87). - NR = 1. Haut. cons. 4,2 cm. Pâte verdâtre, petites inclusions. Pas d'engobe visible.

La cruche de type B.1.2 est, en revanche, isolée.

d/cr.bichr.8 (fig. 92). — NR = 3. Haut. cons. 8,9 cm. Pâte beige, inclusions noires et blanches. Décor peint en "Bichrome amathousien ".

Les deux fragments de cruches à ressaut sur le col (type C.1) sont sans doute résiduels, même si leur décor utilise la technique du « Bichrome amathousien ».

d/cr.bichr.2 (fig. 94). $-\mathrm{NR}=1$. Haut. cons. $7 \mathrm{~cm}$. Pâte beige, inclusions noires et blanches.

\section{3. 3. Amphores}

Les amphores à anses verticales comprennent une petite série de beaux exemplaires de grand format (type A.1) qui n'ont, pour le moment, de parallèle que dans le dépôt de la grotte du sanctuaire.

d/a.bichr.2 (fig. 101). $-\mathrm{NR}=18$. Haut. cons. $18,2 \mathrm{~cm}$; diam. $37,9 \mathrm{~cm}$. Pâte beige, inclusions noires et blanches. Engobe blanchâtre, écaillé par endroit.

d/a.bichr.1 (fig. 102). $-\mathrm{NR}=19$. Haut. cons. $23,4 \mathrm{~cm}$; diam. $38 \mathrm{~cm}$. Pâte verte, friable, inclusions noires et blanches. Engobe beige. Décor peint selon la technique du «Bichrome amathousien ".

d/a.bichr.3 (fig. 103). $-\mathrm{NR}=5$. Haut. cons. $23,6 \mathrm{~cm}$; diam. $30,6 \mathrm{~cm}$. Pâte beige, inclusions noires et blanches. Engobe blanchâtre.

d/a.bichr.4 (fig. 104). - NR = 9. Haut. cons. $25,4 \mathrm{~cm}$; diam. $46 \mathrm{~cm}$. Pâte rose, inclusions noires et blanches. Engobe blanchâtre.

Les amphores à anses de la lèvre à l'épaule possèdent toutes un col nettement évasé, qui invite à les placer dans la classe V (type A.2.3).

70. Deux cruches, l'une provenant de la nécropole d'Amathonte, l'autre issue de fouilles illégales, permettent de compléter le décor qui orne l'épaule de l'exemplaire fragmentaire du rempart Nord ( $B C H 111$ [1987], p. 706, fig. 89 et $B C H 127$ [2003], p. 655, fig. 23) : outre des traits verticaux rouges, on doit restituer des arbres stylisés, ou un thymiatérion, motifs fréquents dans le répertoire du «style d'Amathonte ».

BCH 133 (2009) 
d/a.bichr.5 (fig. 108). $-\mathrm{NR}=1$. Haut. cons. $15,2 \mathrm{~cm}$; diam. $27,6 \mathrm{~cm}$. Pâte beige, inclusions noires et blanches. Engobe blanchâtre.

Les amphorisques du "style d'Amathonte » (type B.3) ont été publiés en détail dans un article précédent ${ }^{71}$.

Un même évasement du col caractérise les amphores de type B.4.1, qui portent un décor géométrique commun dans les productions amathousiennes dès la classe IV.

d/a.bichr.7 (fig. 126). - NR = 4. Haut. cons. $11,8 \mathrm{~cm}$; diam. $21 \mathrm{~cm}$. Pâte beige, inclusions noires et blanches.

d/a.bichr. 8 (fig. 127). - NR = 47. Haut. cons. $28,8 \mathrm{~cm}$; diam. $16,1 \mathrm{~cm}$. Pâte verte, très friable, nombreuses inclusions noires. Engobe blanchâtre, très écaillé. Peintures noire et rouge, parfois difficiles à distinguer. Un tesson, appartenant au bas de la panse, porte un signe peint, difficilement identifiable comme un signe d'écriture ${ }^{72}$.

Huit individus illustrent, enfin, le type des amphores miniatures (B.5), production caractéristique des ateliers amathousiens de la fin de l'archaïsme.

d/a.bichr.19 (fig. 137). - NR = 11. Haut. cons. $12,8 \mathrm{~cm}$; diam. 4,1 cm. Pâte beige, inclusions noires et blanches. Engobe blanchâtre, épais, également passé sur la face interne, sur le haut du col.

d/a.bichr.20 (fig. 138). - NR = 4. Haut. cons. 7,6 cm. Pâte beige, inclusions noires et blanches. Pas d'engobe visible.

\section{3. 4. Jarres}

Cette catégorie n'est représentée que par des exemplaires isolés, de formes variées, à l'exception de la petite série de jarres de type C.3, que caractérisent un même module et un même répertoire décoratif.

Type A.4

d/d.1 (fig. 150). - NR = 5. Haut. cons. $6,6 \mathrm{~cm}$; diam. 19,2 cm. Pâte rose, serrée, petites inclusions noires (non locale).

d/cr.bichr.3 (fig. 151). - NR = 5. Haut. cons. $8,2 \mathrm{~cm}$; diam. $8,5 \mathrm{~cm}$. Pâte beige, petites inclusions noires et blanches. Engobe blanchâtre.

d/d.6 (fig. 158). $-\mathrm{NR}=13$. Haut. cons. $11,2 \mathrm{~cm}$; diam. $15,6 \mathrm{~cm}$. Pâte beige, inclusions noires et blanches. Épais engobe blanc à l'extérieur. Peinture noire et rouge, rehauts de blanc sur la lèvre.

d/d.7 (fig. 159). $-\mathrm{NR}=1$. Haut. cons. $3,4 \mathrm{~cm}$; diam. $14,4 \mathrm{~cm}$. Pâte rose, inclusions noires et blanches.

71. BCH 132 (2008), p. 000.

72. Rappelons toutefois que le dépôt du rempart Nord a livré trois fragments de vase peints avec des inscriptions grecques alphabétiques : P. AUPERT, $B C H 127$ (2003), p. 107-121.

BCH 133 (2009) 
d/d.8 (fig. 160). $-\mathrm{NR}=1$. Haut. cons. $4,6 \mathrm{~cm} ;$ diam. $14,8 \mathrm{~cm}$. Pâte beige, inclusions noires et blanches. Engobe blanchâtre. Deux petits trous ont été percés après cuisson sous la lèvre.

d/d.9 (fig. 161) ${ }^{73} .-\mathrm{NR}=2$. Haut. cons. 5,6 cm; diam. 15,6 cm. Pâte beige, inclusions noires et blanches. Épais engobe beige à l'extérieur.

\section{3. 5. Couvercles}

Les couvercles à profil de courbe convexe et longue lèvre étalée (type A.1.2) sont les plus nombreux.

d/c.2 (fig. 163). - NR = 2. Diam. 14,9 cm. Pâte beige à rose, inclusions noires et blanches. Engobe blanc sur la surface externe.

d/c.3 (fig. 164). - NR = 1. Diam. 16,3 cm. Pâte rose, inclusions noires et blanches.

Un couvercle de type A.2 porte un décor du «style d'Amathonte ».

d/c.7 (fig. 167). - NR = 1. Diam. 16 cm. Pâte beige, petites inclusions noires et blanches. Engobe blanchâtre.

\section{4. RS}

\section{4. 1. Formes ouvertes}

Contrairement aux autres fabriques, ce sont les variantes à lèvre ronde ou légèrement biseautée qui prédominent parmi les bols RS peu profonds à épaule marquée (type A.1).

Type A.1.1

d/b.rs.1 (fig. 2). - NR = 5. Diam. 12,8 cm. Pâte rose, inclusions noires et blanches. Engobe brunrouge, mat.

Type A.1.2

d/b.rs.2 (fig. 5). $-N R=2$. Diam. 19,6 cm. Pâte rose, inclusions noires et blanches. Engobe rouge orangé, mat, très écaillé.

Le type A.2.5, relativement rare parmi les autres fabriques, est illustré par une petite série de bols RS qui, tous, présentent une lèvre à encoche, détail morphologique d'origine levantine.

d/b.rs.4 (fig. 23). - NR = 1. Diam. 10,9 cm. Pâte rose, inclusions noires et blanches. Engobe rouge, mat, très écaillé.

73. Une jarre découverte dans une tombe de la fin de l'archaïsme de Limassol-Aghios Athanasios porte le même décor de feuillage, en blanc sur engobe rouge (technique Bichr.R.). Le rebord du vase, droit et non pas muni d'une courte lèvre étalée, est toutefois différent : P. FlourenTZOS, RDAC 1993, pl. XXXII 120-(1). 
Le type A.4.2 est très proche du précédent, dont il se distingue seulement par un profil rectiligne. Les lèvres à encoche sont, dans cette série également, nombreuses.

d/b.rs.5 (fig. 35). - NR = 3. Diam. 12,1 cm. Pâte ocre, petites inclusions noires. Engobe rouge orangé, lissé et brillant.

Un fragment de lèvre appartient peut-être à un bol de type B.2.1, mais il est de dimensions trop réduites pour que l'identification soit assurée.

Seuls trois individus illustrent le type B.4.2.

d/b.rs.6 (fig. 47). - NR = 1. Diam. $11 \mathrm{~cm}$. Pâte beige, inclusions blanches et noires. Engobe rouge et mat, écaillé par endroit.

Le type B.6.1 est également rare. Le seul exemplaire de profil archéologique complet possède une lèvre légèrement évasée.

d/b.rs.7 (fig. 51). - NR = 11. Diam. 12,1 cm. Pâte orangée, inclusions noires et blanches. Engobe rouge orangé, mat.

Les quelques fragments de lèvre appartenant à des bols à paroi fine (type C) sont trop petits pour que les types puissent être reconstitués avec certitude. Il est probable que ces tessons documentent l'existence de bols à décor de bande rouge sur le haut de la vasque, résiduels dans le dépôt du rempart Nord.

\section{4. 2. Cruches}

Les cruches à ouverture ronde et corps globulaire (type A.1.1) sont souvent de dimensions réduites, voire miniatures. Quelques variantes (position de l'attache supérieure de l'anse, forme de cette dernière, bifide ou en ruban) sont à remarquer.

d/cr.rs.9 (fig. 67). $-\mathrm{NR}=40$. Haut. restituée $16,9 \mathrm{~cm}$. Pâte rose, inclusions noires et blanches. Engobe brun-rouge, épais et mat, coulures à l'intérieur du col. Anse en ruban.

d/cr.rs.6 (fig. 68). - NR = 27. Haut. 15,6 cm. Pâte rose, inclusions noires et blanches. Engobe brun orangé, mat, écaillé par endroit. Anse bifide de section ronde fixée juste sous le col.

d/cr.rs.3 (fig. 69). - NR = 2. Haut. cons. $5,1 \mathrm{~cm}$. Pâte ocre, inclusions noires et blanches. Engobe brun orangé, mat, très écaillé.

Seuls trois individus documentent le type A.4.

d/cr.rs.4 (fig. 80). - NR = 12. Haut. cons. 11,4 cm (sans l'anse). Pâte rose, inclusions noires et blanches. Engobe brun orangé, mat.

Les deux cruches à ouverture pincée entrent dans le type B.1.1.

d/cr.rs.1 (fig. 88). $-\mathrm{NR}=1$. Haut. cons. 3,6 cm (sans l'anse). Pâte marron, inclusions noires et blanches. Engobe rouge orangé, brillant, écaillé par endroit. 


\section{4. 3. Jarres}

Un exemplaire de jarre fragmentaire (type A.5) est peut-être de fabrique RS, mais son état de conservation de permet pas d'en être assuré.

d/d.2. (fig. 152). $-\mathrm{NR}=2$. Haut. cons. $4,7 \mathrm{~cm}$; diam. $16,7 \mathrm{~cm}$. Pâte rose, inclusions noires et blanches. Engobe rouge, mat. Surface couverte de concrétions.

\section{4. 4. Couvercles}

Le fragment de couvercle à profil convexe et lèvre étalée est sans doute, en raison de sa pâte, importé.

d/c.4 (fig. 165). - NR = 2. Diam. 11,6 cm. Pâte brune, très épurée. Surface engobée rouge, lissée et brillante, sur la face interne comme externe.

\section{5. BS}

\section{5. 1. Formes ouvertes}

Létat de conservation des fragments ne permet pas d'assurer l'identification de la fabrique : il est possible, sinon probable, qu'une partie des tessons classés en BS appartienne en réalité à des vases à engobe rouge, sans décor (RS) ou décorés (BoR et Bichr.R.), volontairement noircie (fabriques "Blackened ${ }^{74}$ »). Il est remarquable que toutes les formes répertoriées aient des parallèles exacts en RS, ce qui conforte l'hypothèse. C'est notamment le cas des bols de type A.1.1.

d/b.bs.1 (fig. 3). - NR = 9. Diam. 12,8 cm. Pâte beige, inclusions noires et blanches. Engobe noir, mat. Pas d'anse.

Il en est de même pour les bols A.2.5, à lèvre à encoche, qui reproduisent exactement un type plus commun en RS.

d/b.bs.2 (fig. 24). - NR = 1. Diam. $22 \mathrm{~cm}$. Pâte beige, inclusions noires et blanches. Engobe noir, mat, très écaillé.

Trois fragments de lèvre illustrent peut-être l'existence de bols de type B.2.1, mais l'identification de la forme comme celle de la fabrique sont loin d'être assurées vu l'état de conservation des tessons. Il en est de même pour le seul exemple de bol à lèvre (type B.6.1).

\section{5. 2. Cruches}

Les cruches à ouverture ronde et corps globulaire (type A.1.1) sont nombreuses dans cette fabrique. Elles sont tout à fait semblables aux exemplaires de ce type de fabrique RS, et il est probable qu'un bon nombre d'entre elles, sinon toutes, sont des versions noircies,

74. Pour ces fabriques et leurs ressorts techniques, voir Fl. DANIEL et al. (supra, n. 34), p. 40-44. 
"Blackened", de cruches RS. La prépondérance de cette fabrique " noircie » sur la fabrique originalement rouge fournit un nouvel indice en faveur d'une datation tardive, à la fin du Chypro-Archaïque II, du dépôt du rempart Nord.

d/cr.bs.1 (fig. 70). - NR = 49. Haut. 13,5 cm. Pâte rose, grosses inclusions blanches, et noires plus petites. Engobe brun à noir, mat.

d/cr.bs.6 (fig. 71). - NR = 19. Haut. 14,4 cm. Pâte beige verdâtre, inclusions noires et blanches. Engobe noir mat, très écaillé.

Le type A.3 est plus rare et il n'existe pas en fabrique RS.

d/cr.bs.3 (fig. 77). - NR = 1. Haut. $5 \mathrm{~cm}$. Pâte beige, petites inclusions noires et blanches. Engobe brun à noir, mat, écaillé.

En revanche, le type A.4 reproduit une forme connue en RS.

d/cr.bs.4 (fig. 81). - NR = 2. Haut. cons. 4,6 cm (sans l'anse). Pâte verdâtre, sur-cuite, poreuse. Petites inclusions noires et blanches. Engobe noir, mat et écaillé.

\section{6. BoR}

La fabrique BoR est beaucoup moins commune que dans les contextes chronologiques plus anciens (Chypro-Géométrique III et Chypro-Archaïque I). À la fin de l'archaïsme, elle cède la place à ses variantes polychromes (Bichr.R. et Bichr.R. "Blackened») : il est probable qu'un bon nombre de vases, identifiés comme BoR à partir de l'examen des fragments conservés, appartient en réalité à ces fabriques. Les traitements de surface, moins soignés, offrent un autre repère chronologique : les engobes rouges sont rarement lissés ou polis ; ils sont généralement mats et de teinte brune.

\section{6. 1. Formes ouvertes}

Type A.1.2

d/b.bor.1 (fig. 6). $-\mathrm{NR}=1$. Diam. 24,4 cm. Pâte rose, inclusions noires et blanches. Engobe brun, mat.

Type A.1.3

d/b.bor.2 (fig. 11). $-\mathrm{NR}=14$. Diam. 16,8 cm. Pâte beige, inclusions noires et blanches. Engobe brun, mat.

Type A.2.5

d/b.bor.3 (fig. 25). - NR = 2. Diam. 15,9 cm. Pâte brune, petites inclusions noires et blanches. Engobe rouge lissé, brillant.

Les rares bols de type A.4.2 se rapprochent, par leurs dimensions et leur décor, des bols de ce type de fabrique WP et Bichr.

BCH 133 (2009) 
Les quelques fragments de bols de type B.2.1 sont sans doute résiduels : ils correspondent à des productions de la classe IV (Chypro-Archaïque I) et aucun raccord n'a été retrouvé entre les 10 petits fragments de lèvres, très usés.

d/b.bor.5 (fig. 39). - NR = 1. Diam. 14,3 cm. Pâte beige, inclusions noires et blanches. Engobe brun, mat.

Les bols à lèvre (type B.6.1) illustrent une production tardive dans une fabrique passée de mode $^{75}$. Leur profil écrasé, leur module réduit, l'absence d'anse sont autant d'indices morphologiques de la fin de l'archaïsme.

d/b.bor.7 (fig. 52). - NR = 10. Diam. 10,8 cm. Pâte beige, petites inclusions noires et blanches. Engobe rouge mat.

L'unique fragment de lèvre appartenant à un bol à paroi fine (type C) est probablement résiduel.

\section{6. 2. Cruches}

Les deux individus de type A.3 illustrent l'une des rares formes caractéristique de la classe V en fabrique BoR. On remarquera que l'engobe a partiellement viré au noir, conformément aux tendances décoratives de la fin de l'archaïsme.

d/cr.bor.3 (fig. 78). $-\mathrm{NR}=1$. Haut. cons. 2,6 cm. Pâte beige, serrée, petites inclusions noires. Engobe rouge, brillant, viré au noir sur la majeure partie de la surface.

Les cruches de type B.1.1 présentent également des traits morphologiques propres à la classe $\mathrm{V}$.

d/cr.bor.1 (fig. 89). $-\mathrm{NR}=1$. Haut. cons. $5,1 \mathrm{~cm}$. Pâte marron, petites inclusions noires et blanches. Engobe rouge mat.

Les deux individus qui illustrent le type C.2.2 sont, en revanche, résiduels.

d/cr.bor.4 (fig. 95). — NR = 1. Haut. cons. $3,3 \mathrm{~cm}$. Pâte marron, inclusions noires. Engobe marron, mat.

\section{6. 3. Amphores}

Le fragment d'amphore à anses verticales (type A.2.1) est sans doute également résiduel.

d/a.bor.3 (fig. 106). $-\mathrm{NR}=1$. Haut. cons. $9,4 \mathrm{~cm}$; diam. $28 \mathrm{~cm}$. Pâte ocre, poreuse, nombreuses petites inclusions noires et blanches. Engobe rouge mat.

Les quelques individus illustrant des amphores à col évasé (type B.4.1) sont peutêtre de fabrique Bichr.R., ce que l'état de conservation des fragments ne permet pas de conclure avec certitude.

75. Il est d'ailleurs possible que les quelques fragments inventoriés dans cette fabrique possèdent en réalité un décor Bichr.R., ce que leur état de conservation ne permet pas de reconnaître sûrement.

BCH 133 (2009) 
d/a.bor.4 (fig. 128). - NR = 3. Haut. cons. $6,8 \mathrm{~cm}$; diam. $15 \mathrm{~cm}$. Pâte ocre, poreuse, inclusions noires et blanches. Engobe orangé, mat, très écaillé.

\section{6. 4. Jarres}

Trois jarres fragmentaires entrent dans le type C.1, caractéristique de l'archaïsme avancé.

d/d.4 (fig. 157). $-\mathrm{NR}=1$. Diam. 13,6 cm. Pâte beige, inclusions noires. Engobe rouge mat. Peinture noire.

\section{6. 5. Couvercles}

Mentionnons enfin un couvercle à lèvre étalée :

d/c.1 (fig. 162). - NR = 12. Diam. 12,8 cm. Pâte verdâtre, friable. Petites inclusions noires. Engobe marron, mat, très abîmé.

\section{7. Bichr.R. et Bichr.R. «Blackened »}

Les bols de type A.1, surtout dans leur variante à lèvre biseautée (type A.1.3), sont très communs. Ils portent un décor standardisé, fait de croisillons (détaillés en véritables frises de croix [fig. 13] ou schématisés en simples lignes sécantes [fig. 14]) ou de larges lignes ondulées (fig. 12), rendus en rehauts de peinture blanche. Les mêmes types et les mêmes décors se retrouvent en Bichr.R. «Blackened ». Les exemples à décor interne en fabrique Bichr.R. et décor externe en fabrique "Blackened ", ou vice-versa, sont d'ailleurs fréquents.

d/b.bichr.r.2 (fig. 12). $-\mathrm{NR}=29$. Diam. 15,2 cm. Pâte beige, inclusions noires et blanches. Engobe brun, mat.

d/b.bichr.r.5 (fig. 13). - NR = 9. Diam. 13,2 cm. Pâte rose, inclusions noires et blanches. Engobe rouge lissé.

d/b.bichr.r.6 (fig. 14). $-\mathrm{NR}=8$. Diam. 19,6 cm. Pâte rose, inclusions noires et blanches. Engobe rouge mat, de couleur marron sur la paroi extérieure, du rebord à l'épaule.

d/b.bs.4 (fig. 15). - NR = 20. Diam. 21,5 cm. Pâte rose, inclusions noires et blanches. Engobe marron à noir.

d/b.bs.6 (fig. 16). - NR = 4. Diam. 16,7 cm. Pâte beige, rose au cœur, inclusions noires et blanches. Engobe marron orangé à l'extérieur, marron foncé à l'intérieur.

Comme les précédents, les bols profonds de type B.2.1 sont une adaptation, avec une surface engobée rouge, des séries Bichr. correspondantes. Ils sont toutefois moins nombreux, et ils présentent un mauvais état de conservation, qui interdit souvent une identification précise du décor. Quand ce dernier est lisible, on y reconnaît les frises de chevrons, de croisillons ou de feuilles, caractéristiques du "style d'Amathonte ".

d/b.bichr.r.11 (fig. 40). - NR = 2. Diam. 10,7 cm. Pâte rose, inclusions noires et blanches. Engobe mat et épais, rouge à l'intérieur, marron à l'extérieur. Rehauts de peinture

BCH 133 (2009) 
blanche. Le bol était certainement muni de petites anses horizontales en ruban, qui ne figurent pas sur le dessin, faute d'être conservées.

d/b.bs.7 (fig. 41). - NR = 5. Diam. 16,4 cm. Pâte rose, inclusions noires et blanches. Engobe noirci, gris-noir, mat. Rehauts de peinture blanche. Le bol devait être muni de petites anses horizontales, qui ne sont pas conservées.

Le type B.6.1 est rare, en Bichr.R. comme dans les autres fabriques. On notera toutefois qu'un bol de plus grandes dimensions, muni d'anses (d/b.bichr.r.9, fig. 54), complète cette série, généralement déclinée en miniature. Les exemplaires de fabrique Bichr.R. "Blackened» sont plus fréquents que ceux à surface rouge.

d/b.bichr.r.10 (fig. 53). $-\mathrm{NR}=6$. Diam. 11,8 cm. Pâte rose, inclusions noires et blanches, engobe brun-rouge, mat. Rehauts de peinture blanche.

d/b.bichr.r.9 (fig. 54). — NR = 1. Diam. 20,4 cm. Même pâte et même décor.

\section{7. 1. Amphores}

L'existence d'au moins une amphore de type A.1 est assurée par la découverte d'une anse trifide (fig. 105).

Quelques amphorisques Bichr.R. (fig. 117) documentent un type (B.3), qui est surtout décliné dans des fabriques " noircies " (Bichr.R. « Blackened»).

d/a.bichr.r.3 (fig. 117). - NR = 3. Haut. cons. $7,6 \mathrm{~cm}$; diam. $9,8 \mathrm{~cm}$. Pâte rose, inclusions noires et blanches. Engobe brun rouge, mat, écaillé. Rehauts de peinture blanche.

d/a.bs.3 (fig. 118). - NR = 34. Haut. cons. $19,1 \mathrm{~cm}$; diam. $11,2 \mathrm{~cm}$. Pâte beige, inclusions noires et blanches. Engobe rouge viré au noir, mat. À l'extérieur, le haut du col n'est pas engobé (technique WP).

d/a.bs.2 (fig. 119). $-\mathrm{NR}=32$. Haut. cons. $23,9 \mathrm{~cm}$; diam. $13 \mathrm{~cm}$. Même pâte. Engobe rouge viré au noir. L'ensemble du vase est engobé et décoré avec des rehauts de peinture blanche, en plus de la peinture noire.

Les amphores à col évasé et lèvre en bourrelet externe forment une petite série, qui est aussi déclinée, mais plus rarement, en fabrique " noircie " (Bichr.R. "Blackened»).

d/a.bichr.r.5 (fig. 129). - NR = 9. Haut. cons. $9,8 \mathrm{~cm} ;$ diam. 26,4 cm. Pâte beige à rose, inclusions noires et blanches. Engobe rouge orangé, mat. La surface extérieure est très abîmée et ne permet pas de restituer sûrement le décor du col.

d/a.bichr.r.6 (fig. 130). $-\mathrm{NR}=9$. Haut. cons. $12,2 \mathrm{~cm}$; diam. 15,6 cm. Pâte rose, inclusions noires et blanches. Engobe rouge mat, très écaillé.

d/a.bs.9 (fig. 131). - NR = 6. Haut. cons. $9,1 \mathrm{~cm}$; diam. $14,2 \mathrm{~cm}$. Pâte verdâtre, petites inclusions noires et blanches. Le haut du col est décoré, à l'extérieur, en peinture noire sur surface réservée (technique WP). Le reste du vase est recouvert d'un engobe rouge, viré au noir, qui sert de support au décor peint en noir.

d/a.bs.10 (fig. 132). - NR = 11. Haut. cons. $16,3 \mathrm{~cm}$; diam. $30,7 \mathrm{~cm}$. Pâte rose, inclusions noires et blanches. Le haut du col est décoré, à l'extérieur, en peinture noire et rouge sur un épais engobe blanc (technique Bichr.). Le reste du vase porte

BCH 133 (2009) 
un engobe rouge, mat, qui a viré au noir, et qui sert de support à un décor peint en noir et blanc.

Les amphores de type B.4.2, qui n'existent que dans cette fabrique, ne sont pas de fabrication locale.

d/a.bichr.r.7 (fig. 133). - NR = 108. Haut. $51,5 \mathrm{~cm}$; diam. 21,6 cm. Pâte beige, grise au cœur, petites inclusions noires et blanches. Engobe orangé, mat. Peinture noire et rouge.

d/a.bs.7 (fig. 134). $-\mathrm{NR}=4$. Haut. cons. $6 \mathrm{~cm}$; diam. $15,8 \mathrm{~cm}$. Pâte grise, petites inclusions noires et blanches. Engobe noir bleuté.

d/a.bs.5 (fig. 135). - NR = 1. Haut. cons. 11,2 cm; diam. 29,1 cm. Même pâte et même engobe.

\section{7. 2. Jarres}

Un fragment illustre le type B.2.

d/d.3 (fig. 155). $-\mathrm{NR}=1$. Diam. $26,8 \mathrm{~cm}$. Pâte beige, inclusions blanches et noires. Engobe rouge, peinture noire et rehauts de blanc.

\section{7. 3. Couvercles}

Je n'ai identifié qu'un fragment de couvercle Bichr.R.

d/c.6 (fig. 166). - NR = 9. Diam. $22,8 \mathrm{~cm}$. Pâte beige, très épurée, probablement non amathousienne. Surface engobée marron, lissée et brillante, sur la face interne comme externe. Rehauts de blanc, en plus de la peinture noire, sur la face externe.

\section{7. 4. Fabriques hybrides}

Cette catégorie, qui regroupe des vases décorés selon différentes techniques - et qui sont par conséquent attribuables simultanément à différentes fabriques de la classification suédoise - comprend un nombre très limité de formes ${ }^{76}$. Il s'agit essentiellement d'amphores à anses horizontales, de type B.1.1.
d/a.bor.2 (fig. 109). - NR = 3. Haut. cons. $11 \mathrm{~cm}$; diam. $30 \mathrm{~cm}$. Pâte rouge, grise au cœur. Engobe rouge mat. Le col, dont la surface extérieure n'est pas engobée, est décoré selon la technique Bichr.
d/a.bichr.r.4 (fig. 110). $-\mathrm{NR}=4$. Haut. cons. $13 \mathrm{~cm}$; diam. $28,5 \mathrm{~cm}$. Pâte beige, inclusions noires et blanches. Engobe brun rouge, mat. Le col, dont la surface extérieure n'est pas engobée, est décoré selon la technique WP.

76. Quelques spécimens Bichr.R. entrant dans d'autres types portent parfois un décor hybride, le haut du col étant, à l'extérieur, réservé et non pas engobé (amphores des types B.3 et B.4.1, fig. 118 et 131-132). Il s'agit toutefois, en l'occurrence, d'une simple variante décorative et non pas d'une série cohérente, du point de vue de la forme et de la technique de décor, comme c'est le cas pour les amphores de type B.1.1.

BCH 133 (2009) 
On y ajoutera une jarre isolée et fragmentaire, dont le décor peut être restitué grâce à un exemplaire identique provenant des nécropoles 77 .
d/d.12 (fig. 149). $-\mathrm{NR}=1$. Haut. cons. $3,7 \mathrm{~cm}$; diam. $31 \mathrm{~cm}$. Pâte beige, inclusions noires et blanches. Le haut du col est peint, à l'extérieur, en noir sur surface blanche (technique WP) ; sur le reste du vase et à l'intérieur du col, en noir, et peut- être aussi en blanc, sur engobe rouge (technique BoR ou Bichr.R.). Le décor à l'intérieur du col est trop mal conservé pour être dessiné.

\section{LES IMPORTATIONS LEVANTINES}

Les seules importations levantines sûrement identifiées sont des jarres-torpilles. Comme celui des amphores à anses de panier, le dossier des amphores commerciales levantines est vaste et difficile, et il excède largement les limites imposées à la publication de ce dépôt. Les exemplaires mis au jour contre le rempart Nord s'intègrent dans la typologie sommaire proposée pour l'étude du matériel du sanctuaire ${ }^{78}$ : sans aller plus loin dans les identifications, reconnaissons donc l'homogénéité de date, et peut-être de provenance, des amphores déposées dans ces deux contextes.

Les jarres-torpilles sont des vases de transport, dont la forme est héritière des jarres cananéennes du Bronze Récent. Elles constituent, au sanctuaire, la quasi-totalité des importations; elles sont accompagnées, au palais, par des amphores commerciales et de la céramique fine grecques. Il est probable que, dans la plupart des cas, ces amphores transportaient du vin. Leur provenance est encore difficile à définir, la variété des pâtes invite à supposer plusieurs centres différents, et il n'est pas exclu que certaines d'entre elles aient pu être produites à Chypre. Aucune, toutefois, n'est de fabrication amathousienne.

Seul le type B, défini pour le matériel du sanctuaire, et caractérisé par une épaule carénée et non plus arrondie, est représenté dans le dépôt. Cela ne doit pas surprendre : le type A est propre aux contextes du début de l'époque archaïque et, au sanctuaire, il n'est attesté que parmi les amphores du bothros.

\begin{tabular}{l|c|c} 
Type & Rempart Nord & Grotte \\
\hline \hline B.1 & NMI & NMI \\
\hline B.2 & 112 & 55 \\
\hline TOTAL & 19 & 5 \\
\hline \hline
\end{tabular}

77. $B C H 133$ (1989), p. 810, fig. 78.

78. Amathonte VI, p. 82-83.

BCH 133 (2009) 
Tableau 10. - Répartition des jarres-torpilles. Comparaison entre les dépôts du rempart Nord et de la grotte du sanctuaire.

\section{B. SANS COL (fig. 168-175)}

\section{B.1. Lèvre ronde redressée (fig. 168-173)}

d/t.1 (fig. 168). $-\mathrm{NR}=25$. Haut. cons. $26,7 \mathrm{~cm}$; diam. $9 \mathrm{~cm}$. Pâte grise, poreuse, nombreuses inclusions blanches. Surface extérieure rouge orangé.

d/t.2 (fig. 169). - NR = 1. Haut. cons. $13 \mathrm{~cm} ;$ diam. $9,7 \mathrm{~cm}$. Pâte beige à rose, petites inclusions noires. Surface extérieure beige.

d/t.3 (fig. 170). - NR = 3. Haut. cons. $5,4 \mathrm{~cm}$; diam. $9,9 \mathrm{~cm}$. Pâte blanchâtre, friable, petites inclusions noires. Surface extérieure blanchâtre.

d/t.4 (fig. 171). - NR = 5. Haut. cons. $13 \mathrm{~cm}$; diam. $8,5 \mathrm{~cm}$. Pâte blanchâtre, friable, petites inclusions noires. Surface extérieure blanchâtre.

d/t.5 (fig. 172). $-\mathrm{NR}=15$. Haut. cons. $16 \mathrm{~cm}$; diam. 10,9 cm. Pâte brune, inclusions noires et marron. Surface marron.

d/t.6 (fig. 173). - $\mathrm{NR}=65$. Haut. cons. $27,6 \mathrm{~cm}$; diam. $9,8 \mathrm{~cm}$. Pâte beige à rose, très friable, inclusions noires, blanches et marron. Surface beige orangé.

\section{B.2. Lèvre repliée (fig. 174-175)}

d/t.7 (fig. 174). - NR = 2. Haut. cons. $7 \mathrm{~cm}$; diam. 9,6 cm. Pâte beige, inclusions noires, brunes et blanches. Surface extérieure blanchâtre.

d/t.8 (fig. 175). $-\mathrm{NR}=1$. Haut. cons. $2 \mathrm{~cm}$; diam. $9 \mathrm{~cm}$. Pâte beige, petites inclusions noires et brunes. Surface extérieure blanchâtre.

Aucun profil archéologique complet n'a pu être restitué, mais un certain nombre de fonds viennent compléter la catégorie des jarres-torpilles (fig. 176-178). Les formes en sont variées, mais les fonds pointus, caractéristiques des types de l'archaïsme récent, prédominent.

\section{COMMENTAIRE}

La publication récente de plusieurs lots de céramique archaïque a permis de mettre en évidence les caractères particuliers de la production amathousienne : le matériel du dépôt du rempart Nord en confirme les tendances principales, et il en offre de nouvelles illustrations. Deux aspects de cet assemblage remarquable, et par sa qualité et par sa quantité, doivent être distingués. Le premier est lié à sa datation : les vases constitutifs du dépôt sont représentatifs des productions amathousiennes de la fin de l'époque archaïque, c'est-à-dire du moment où les ateliers du royaume font preuve d'une inventivité et d'une

BCH 133 (2009) 
originalité sans précédent, qui culmine avec la création du "style d'Amathonte ». L'autre aspect est lié au contexte : les vases du dépôt proviennent selon toute probabilité du palais de l'acropole et ils offrent donc, pour la première fois, une documentation primaire sur le mobilier d'un palais chypriote de la fin de l'archaïsme. Si donc, sous son premier aspect, le matériel publié dans cet article trouve de nombreuses correspondances parmi les découvertes du sanctuaire et des nécropoles, il consiste, sous son second aspect, en un assemblage singulier, où la présence de certains types rares ou la proportion de certaines catégories éclairent des pratiques propres au palais.

\section{1. LA CHRONOLOGIE : LA CÉRAMIQUE AMATHOUSIENNE DE LA FIN DE L'ARCHAÏSME}

Le matériel du dépôt est, dans ses caractéristiques morphologiques et décoratives, exemplaire d'un assemblage céramique d'Amathonte à la fin de l'archaïsme. Les traits en sont désormais connus et je me contenterai ici d'en souligner les tendances principales ${ }^{79}$.

À l'exception de quelques vases résiduels, la majorité des types attestés dans le dépôt sont attribuables à la classe V, voire, pour certains d'entre eux, VI. C'est, par exemple, le cas des cruches de type A.4 (fig. 79-81) dont la présence n'est pas documentée dans les tombes avant la fin du VIe s. av. J.-C. Les formes sont caractérisées par un profil écrasé : les corps deviennent plus biconiques que globulaires, les cols sont évasés ${ }^{80}$, les anses horizontales des amphores ont tendance à s'écarter de la paroi, les anses verticales des cruches deviennent débordantes. Une certaine simplification touche les formes : les cruches à arête sur le col, d'origine levantine, disparaissent du répertoire et la plupart des vases à verser possèdent un profil simple, à ouverture ronde ou légèrement pincée, sans nette distinction entre le col proprement dit et la lèvre (cruches de type B.1.1, fig. 82-89), autant de traits qui annoncent le répertoire d'époque classique. Cet écrasement des formes entraine l'apparition d'un bon nombre d'exemplaires de dimensions réduites ou même miniatures : leur présence, qui est également nombreuse dans les tombes contemporaines ou au sanctuaire, me paraît, en effet, liée à une évolution générale des formes, et non pas à des motifs d'ordre cultuel. Cette tendance miniaturiste touche des formes connues dès la classe IV (bols de type A.1.3, fig. 9-16 ou A.3.2, fig. 28, cruches RS de type A.1.1, fig. 69) et elle suscite la naissance de nouveaux types (amphorisques de type B.3, fig. 116-119 ou B.5, fig. 136-138, bols de type B.5.2, fig. 48). Si les modèles levantins disparaissent du répertoire des cruches, ils influencent celui des formes ouvertes où les types sans anses, de dimensions réduites et à lèvre parfois marquée d'un ressaut, se multiplient, en fabriques peintes (fig. 46) et, surtout, en PW (type A.2.1, fig. 17-18 et 20, type A.4.1, fig. 29-31).

79. Voir en particulier Amathonte VI, p. 84-90 et S. FOURRIER, CCEC 35 (2005), p. 55-76.

80. Les amphores de type B.4 sont ainsi largement majoritaires. 
De fait, autre signe des temps, la fabrique PW est la plus commune et elle supplante les fabriques à décor peint. Les fabriques à engobe rouge du Chypro-Géométrique III et de l'Archaïque I, RS et BoR, sont caractérisées par des traitements de surface moins soignés (engobe mat et non plus lissé) et elles tendent à être supplantées par la fabrique Bichr.R., souvent déclinée dans sa version noircie, "Blackened ». Parallèlement, la fabrique Bichr. connaît une dernière période de vogue, avec des décors souvent chargés et colorés. Les vases sont parfois recouverts d'un épais engobe blanchâtre, qu'il s'agisse de bols (fig. 22), d'amphores (fig. 103-104) ou de jarres (fig. 158). Sur certains exemples, le décor n'est rendu qu'en peinture rouge (fig. 76); sur d'autres, des rehauts de blanc complètent le noir et le rouge (technique Polychrome, fig. 22 ou 158). Enfin, les artisans jouent volontiers sur les techniques de décor traditionnelles ${ }^{81}$, soit en les mêlant (techniques hybrides), soit en les transformant par cuisson réductrice (technique du "Bichrome amathousien»). Les schémas décoratifs s'enrichissent également, les traditionnels motifs géométriques étant complétés de végétaux et, parfois, de représentations figurées. Le répertoire du "style d'Amathonte ", qui trouve sa plus belle expression dans la série des amphorisques ${ }^{82}$, est décliné, sous une forme plus ou moins simplifiée, sur d'autres types de récipients, bols (fig. 38 et 46), amphores (fig. 104), jarres (fig. 151 et 158-161), couvercles (fig. 167). Il est remarquable que ce style typiquement local, qui reprend des tendances stylistiques propres aux ateliers de l'Est de l'île, le fasse avec un certain décalage, ce répertoire végétal et figuré étant en vogue, dans la région de Salamine notamment, au cours du ChyproArchaïque I ${ }^{83}$.

\section{2. LE CONTEXTE : LE MOBILIER CÉRAMIQUE DU PALAIS}

La comparaison avec l'assemblage céramique contemporain de la grotte du sanctuaire met en relief des différences, liées à la fonction et à l'origine des vases, bref, à leur contexte.

Comme au sanctuaire, l'écrasante majorité du matériel est de production locale. Mais les importations sont plus nombreuses au palais et, surtout, d'origine plus variée. Ainsi, on n'a pratiquement pas recueilli d'importation grecque archaïque dans le sanctuaire, alors qu'elles sont documentées dans le dépôt du rempart Nord, essentiellement par des amphores commerciales et de la vaisselle à boire ${ }^{84}$. Aucune céramique fine d'origine levantine n'a été sûrement identifiée, mais on retrouve, au palais comme au sanctuaire, des

81. Pour les fabriques hybrides : L. AlPE, S. Fourrier (supra, n. 42), p. 149-167. Pour une étude détaillée des techniques décoratives à l'époque archaïque : Fl. DANIEL (supra, n. 34), p. 37-65.

82. BCH 132 (2008), p. 000. Ces derniers sont le plus souvent recouverts d'un épais engobe blanc, et ils portent généralement un décor Polychrome, avec des rehauts de blanc sur la peinture noire.

83. Voir M. Yon (supra, n. 39), p. 35-54.

84. En attendant la publication des importations grecques du dépôt par B. Blandin, on se reportera à l'aperçu sur les découvertes du palais de l'acropole, publié par Th. PETIT, RA 1995, p. 211-223.

BCH 133 (2009) 
amphores commerciales (jarres-torpilles). Le répertoire levantin influence, par ailleurs, la forme de certains bols sans anses (type A.2.5, fig. 21, 22 et 25 ; type A.4.1, fig. 29-31), dont quelques-uns sont peut-être de production non-amathousienne (plutôt de Kition que du continent). Les importations d'autres régions de l'île sont rares : les quatre amphores de type B.4.2 (fig. 133-135) et une jarre (fig. 150) proviennent sans doute de Paphos ; une cruche à pastille rapportée (fig. 82) renvoie aux corpus de Salamine ou d'Idalion ; des vases isolés, jarre (fig. 153) ou couvercles (fig. 165-166), présentent une pâte proche de celle des productions de Kition.

Je me suis intéressée, dans des articles précédents ${ }^{85}$, à deux catégories qui me paraissaient particulièrement remarquables, parmi le matériel du dépôt, les céramiques culinaires et les vases du "style d'Amathonte ». Or, ces deux catégories sont en quelque sorte exemplaires de ce qui distingue l'assemblage du rempart Nord de celui, contemporain, de la grotte du sanctuaire : la présence de vases " utilitaires " (sans décor, utilisés pour le transport, le stockage ou la préparation de denrées) et celle de vases « beaux " (dont le décor puise dans le répertoire du "style d'Amathonte »). Parmi les céramiques du premier groupe, on trouve des formes attestées par ailleurs, mais rares au sanctuaire : amphores à anses de panier (type C, fig. 139-148) ; cuvettes (type D, fig. 59-65). On trouve également des vases de grandes dimensions, le plus souvent de fabrique PW, qui entrent dans des types connus ou qui sont inédits : amphores pithoïdes de type A.2.2 (fig. 107), B.2.1 (fig. 111-112), B.2.3 (fig. 115) ; jarres PW de type B.2 ou C.1 (fig. 154 et 156). Les céramiques du second groupe comprennent également des vases de grand format (amphores de type A.1, fig. 99-105), mais surtout des formats réduits (amphorisques, fig. 117-119, jarres, fig. 151, 158-161 et bols, fig. 9-16, 36-41, 46). Ce sont, de fait, les vases à boire qui présentent le plus de variété : issus du répertoire égéen et richement décorés, ils sont munis d'anses; d'inspiration levantine et de fabrique monochrome (RS et surtout PW, fig. 17-18 et 20), ils en sont dépourvus ${ }^{86}$. Les dépôts de fondation, fouillés au palais de l'acropole, associent justement des marmites à de petits bols $\mathrm{PW}{ }^{87}$. On remarquera que les bols à paroi fine (type $\mathrm{C}$ ), qui constituent la forme de vase à boire d'origine levantine la mieux représentée au sanctuaire, sont peu nombreux au palais, peut-être remplacés par des vases plus précieux, importés de Grèce.

L'assemblage céramique du palais d'Amathonte à la fin de l'archaïsme, tel, du moins, qu'on peut le reconstituer à partir des découvertes du rempart Nord, indique donc que le palais était un lieu où arrivaient des denrées (amphores commerciales grecques, levantines

85. BCH 131 (2007), p. 67-93; BCH 132 (2008), p. 000.

86. Sur cette concurrence, dans le répertoire des vases à boire chypriotes, de ces deux modèles, voir S. Fourrier, "East Greek and Cypriote Ceramics of the Archaic Period ", dans V. KarageOrGHIS, O. Kouka (éds), Cyprus and the East Aegean. Intercultural Contacts from 3000 to 500 BC (2009), p. 131-132.

87. Th. PETIT, BCH 113 (1989), p. 135-148. 
et locales) et où elles étaient stockées (amphores pithoïdes). C’est aussi là qu'elles étaient préparées (céramiques culinaires, cuvettes) et consommées (nombreuses formes ouvertes, dont beaucoup sont des vases à boire). Certainement, le banquet collectif était l'une des activités pratiquées au palais, et l'importance, au sein du dépôt, d'un mobilier céramique lié au vin et à sa consommation 88 , en est l'un des indices les plus probants. Le poids social de cette pratique apparaît dans le goût pour des produits (amphores commerciales) et des récipients (skyphoi) importés; il explique aussi que des vases " utilitaires " (céramiques culinaires, «bols perses ») soient déposés dans des tombes royales, comme à Salamine.

88. Peut-être faut-il ranger dans cette catégorie les " bols perses " : les marques d'usure qu'ils portent sont peut-être dus à la réduction en poudre d'épices et d'aromates qu'on versait dans le vin plutôt qu'au broyage de céréales. Le mortier fait, en tout cas, partie des ustensiles de cuisine indispensables à la préparation du banquet : Athénée, Deipnosophistes IV 169b-c.

BCH 133 (2009) 
1

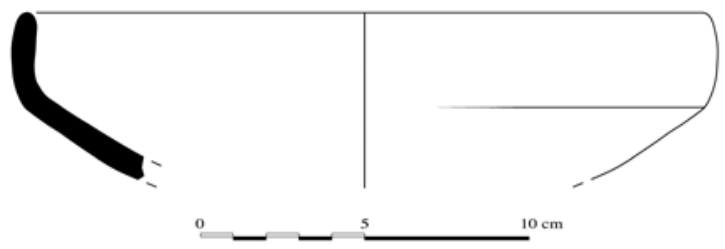

2

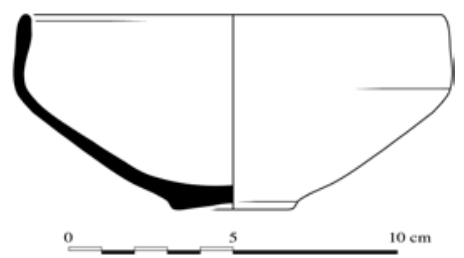

3

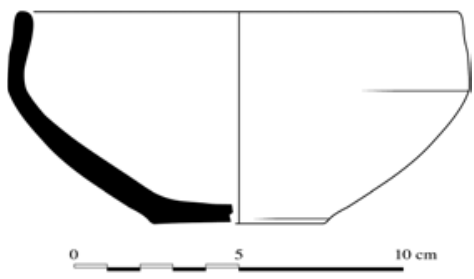

4
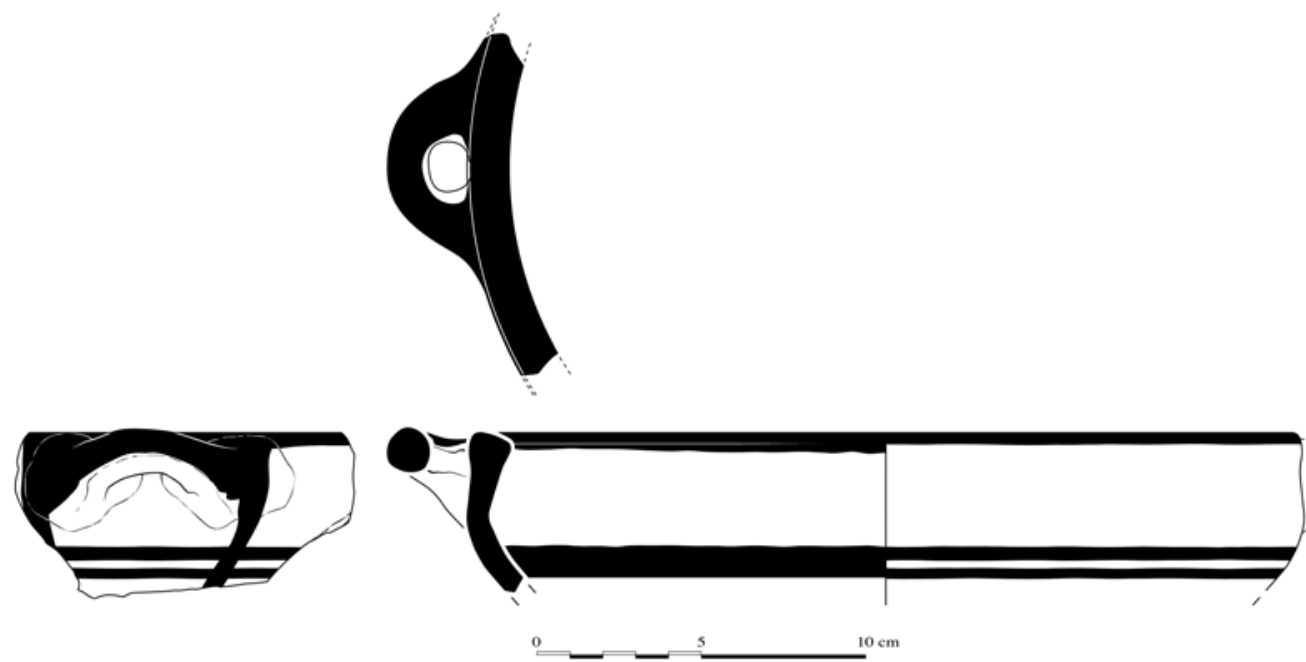

Fig. 1. - d/b.pw.10 (dessin EFA, J. Humbert).

Fig. 2. - d/b.rs.1 (dessin EFA, J. Humbert).

Fig. 2. - d/b.bs.1 (dessin EFA, J. Humbert).

Fig. 4. — d/b.wp.4 (dessin EFA, J. Humbert). 
5

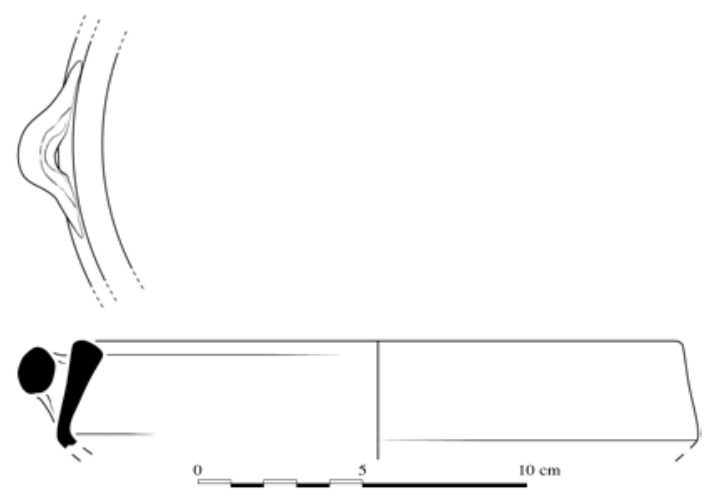

6

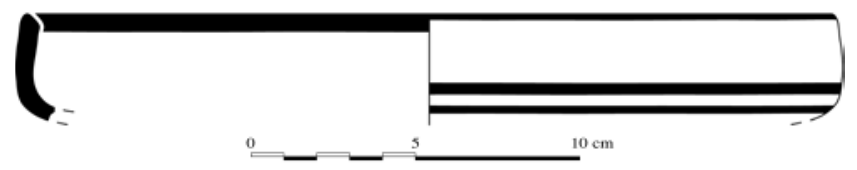

7
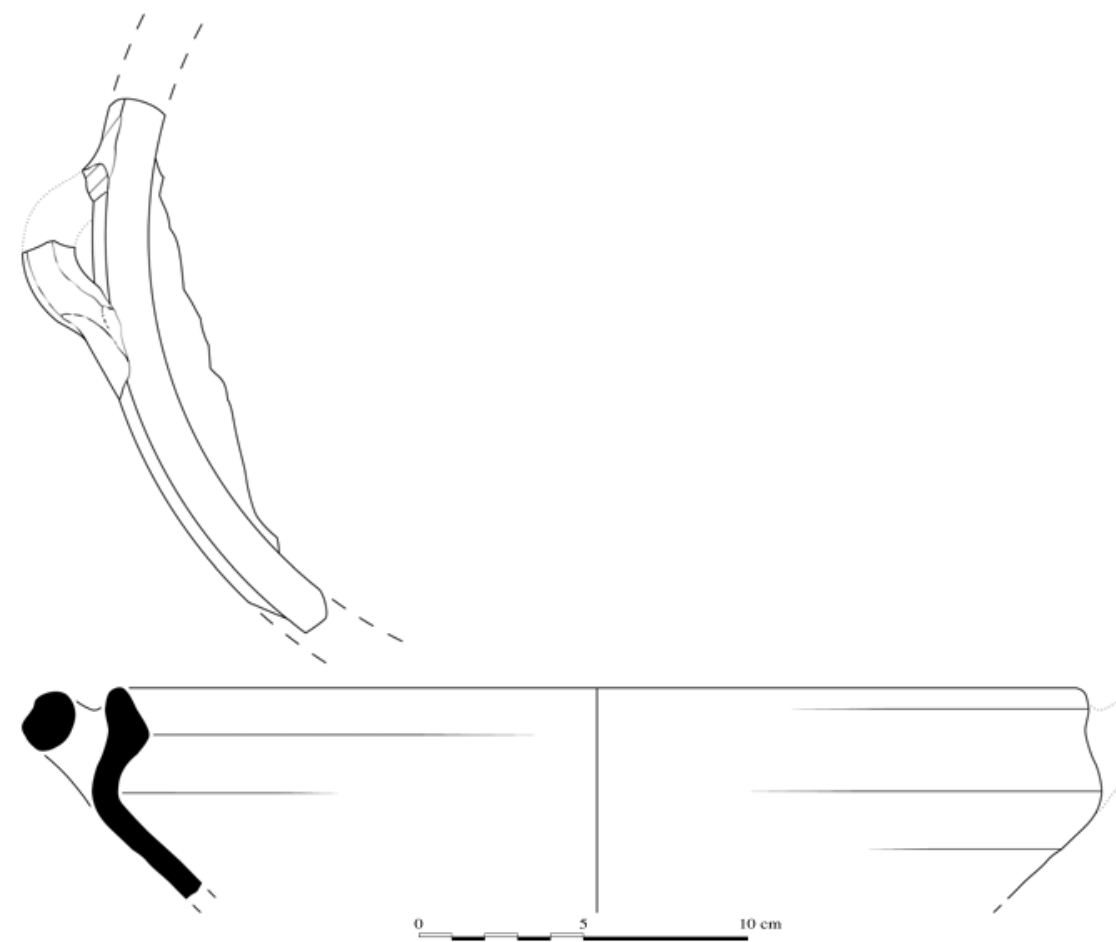

Fig. 5. - d/b.rs.2 (dessin EFA, J. Humbert).

Fig. 6. - d/b.bor.1 (dessin EFA, J. Humbert).

Fig. 7. — d/b.pw.17(dessin EFA, J. Humbert).

BCH 133 (2009) 

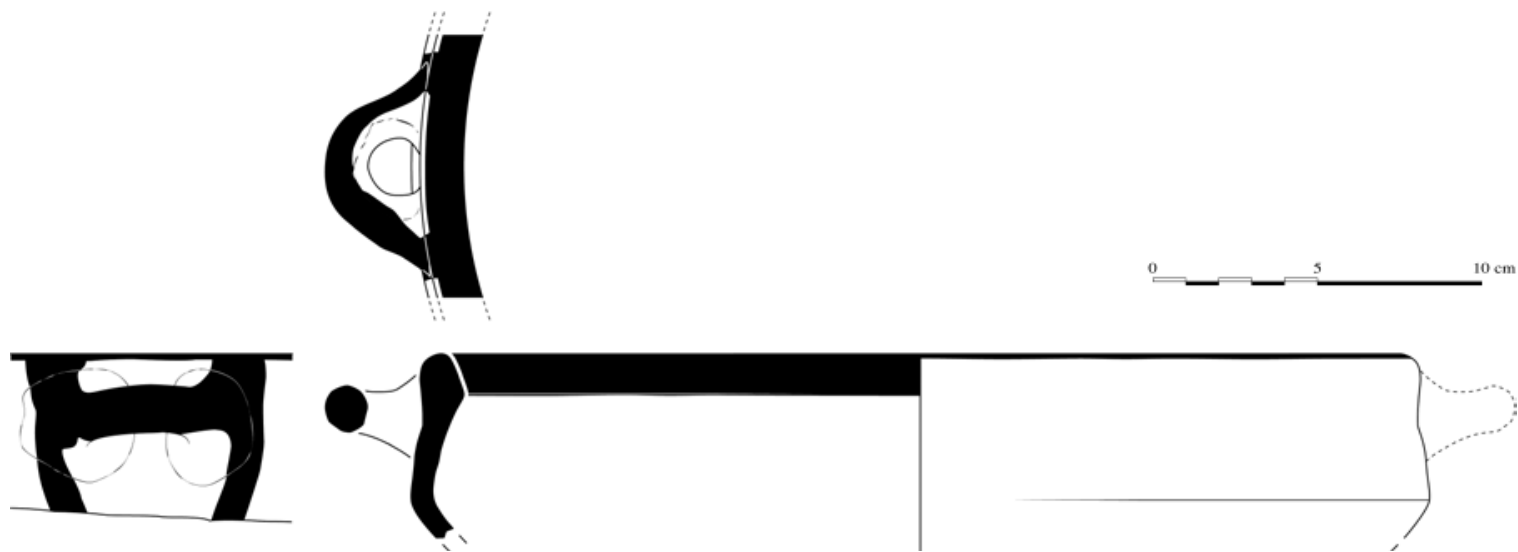

9
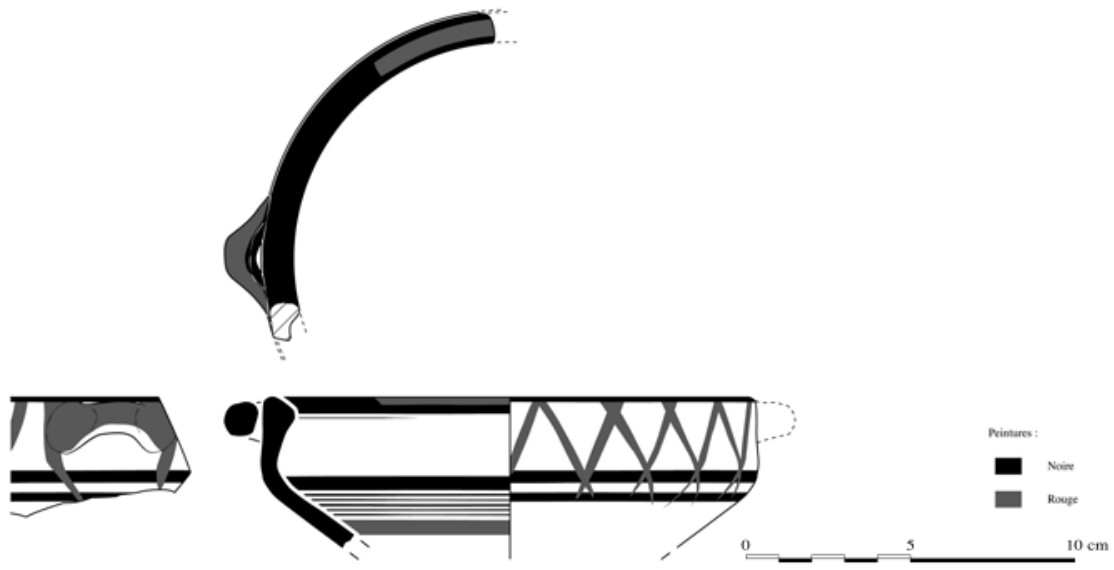

10

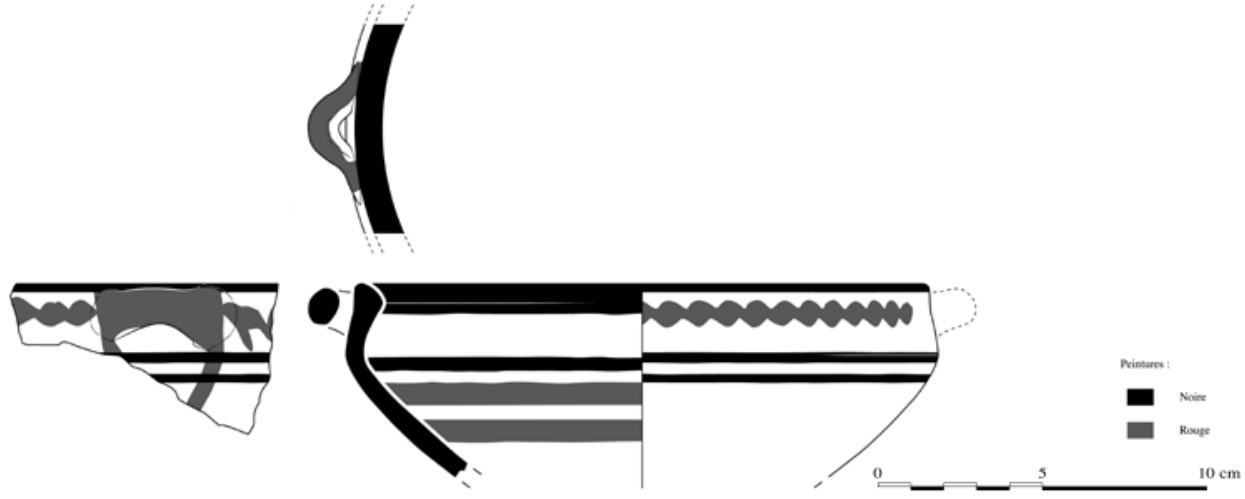

Fig. 8. - d/b.wp.5 (dessin EFA, J. Humbert).

Fig. 9. - d/b.bichr.3 (dessin EFA, J. Humbert).

Fig. 10. - d/b.bichr.5 (dessin EFA, J. Humbert). 
11

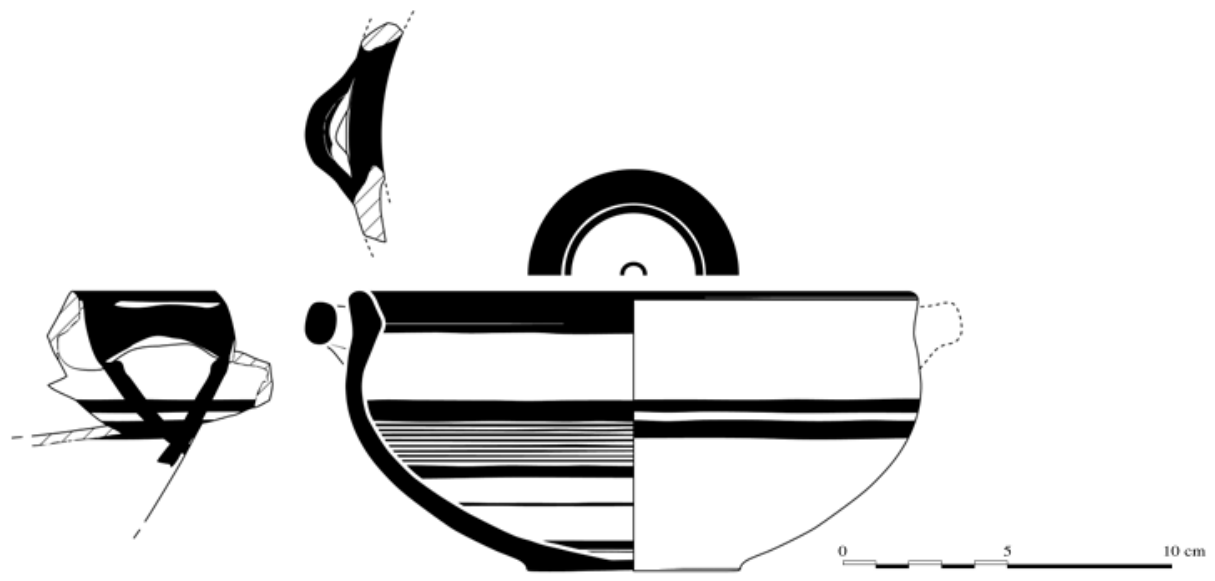

12
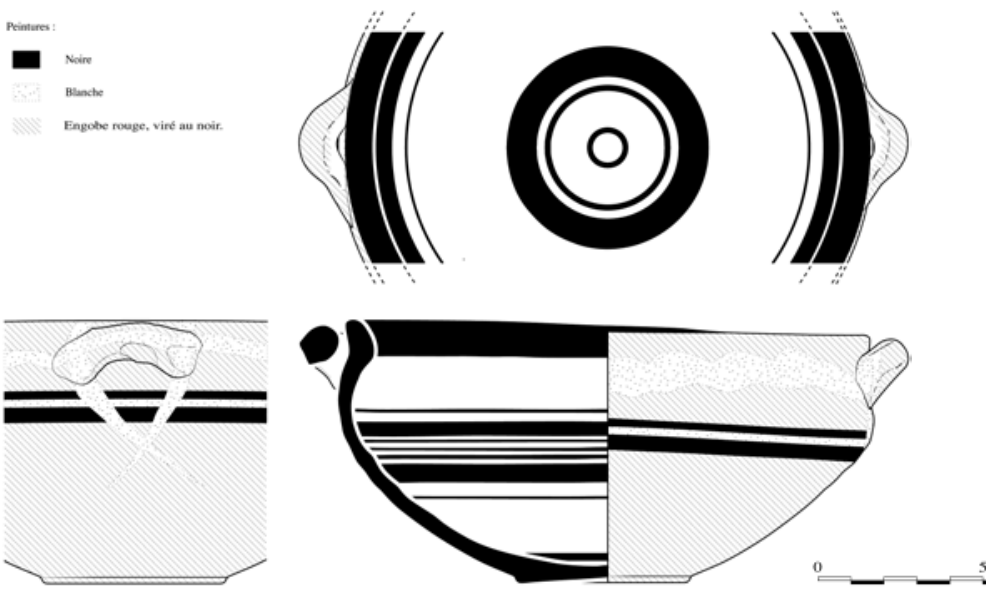

13

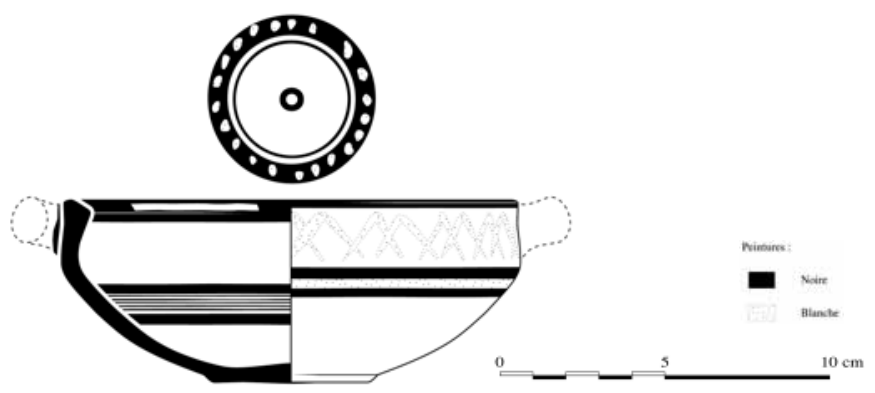

Fig. 11. - d/b.bor.2 (dessin EFA, J. Humbert).

Fig. 12. — d/b.bichr.r.2 (dessin EFA, J. Humbert).

Fig. 13. — d/b.bichr.r.5 (dessin EFA, J. Humbert).

BCH 133 (2009) 
14

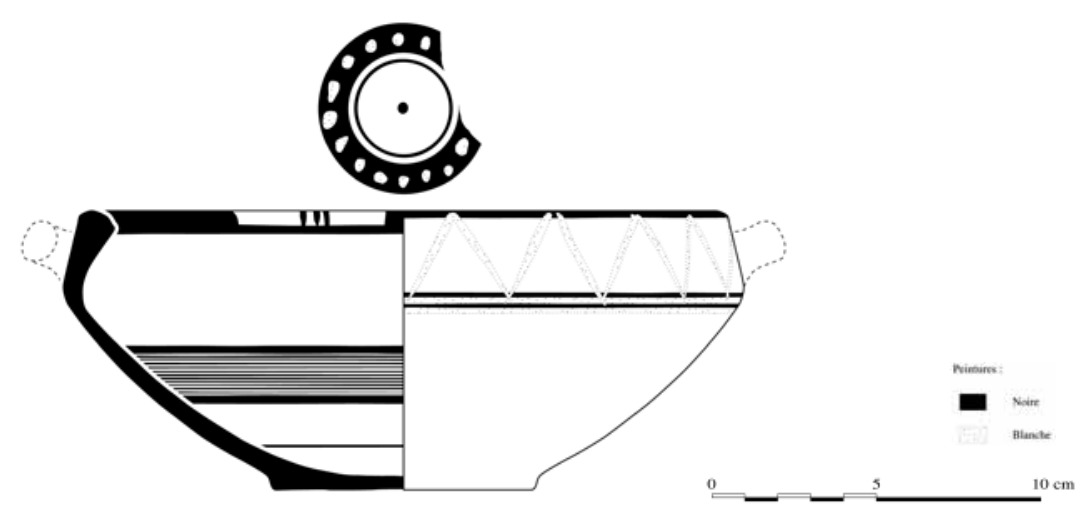

15
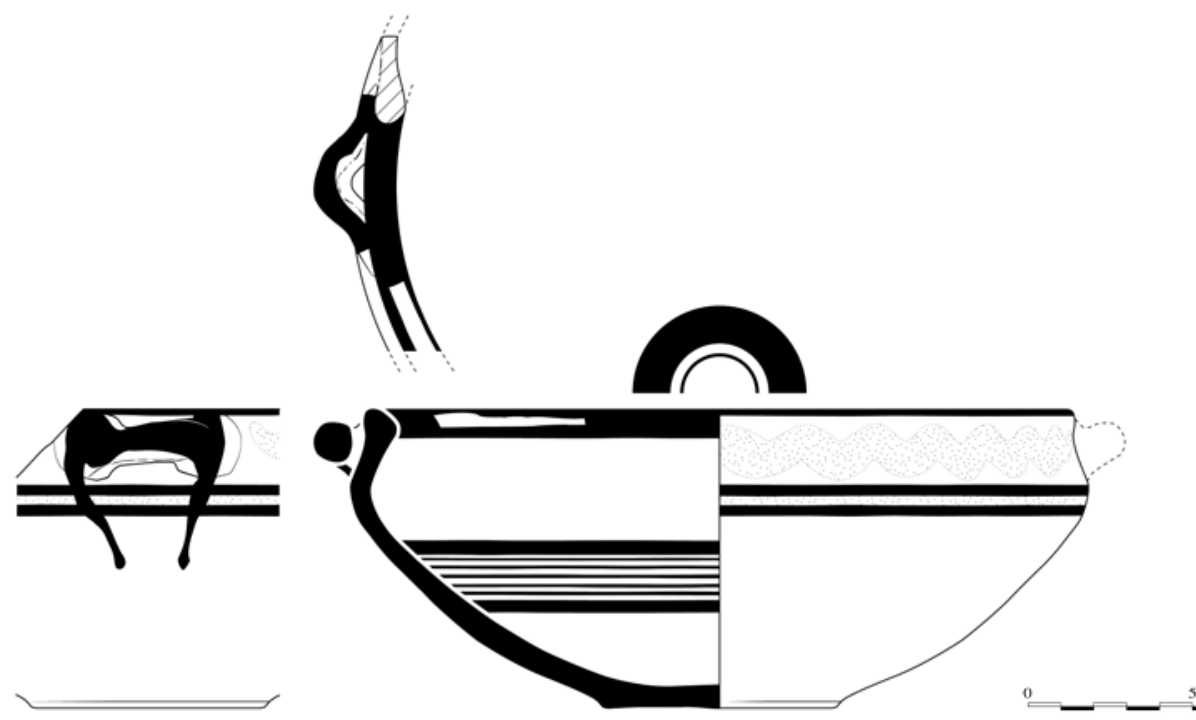

Fig. 14. — d/b.bichr.r.6 (dessin EFA, J. Humbert).

Fig. 15. - d/b.bs.4 (dessin EFA, J. Humbert). 
16
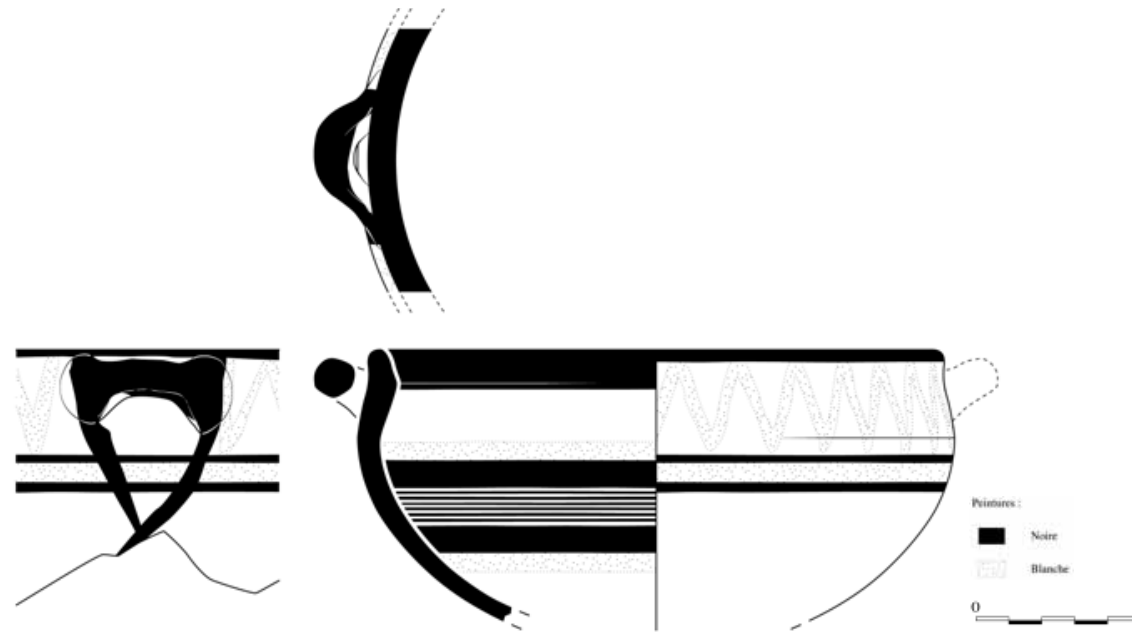

17

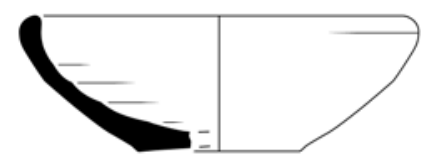

18

19
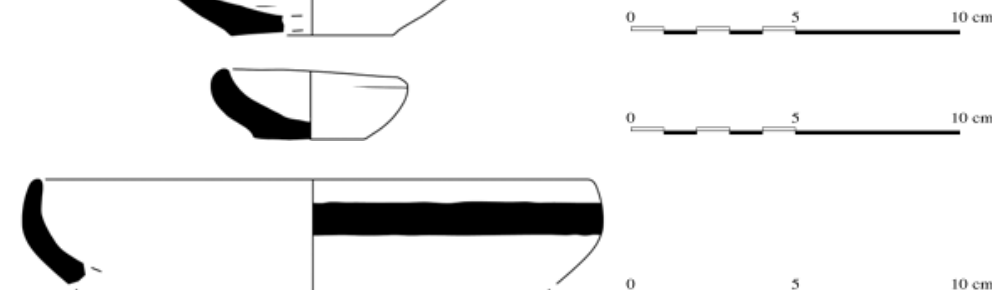

20
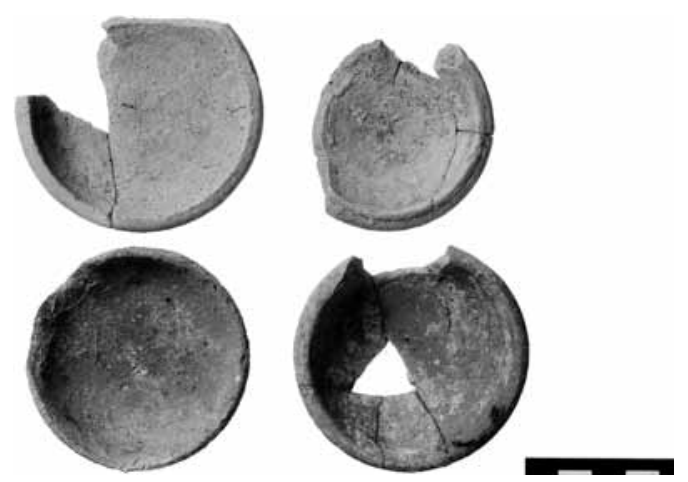

Fig. 16. — d/b.bs.6 (dessin EFA, J. Humbert).

Fig. 17. — d/b.pw.22 (dessin EFA, J. Humbert).

Fig. 18. - d/b.pw.26 (dessin EFA, J. Humbert).

Fig. 19. — d/b.wp.7 (dessin EFA, J. Humbert).

Fig. 20. - Bols PW de type A.2.1 (cl. EFA, Ph. Collet).

BCH 133 (2009) 
21

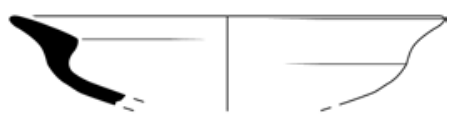

22

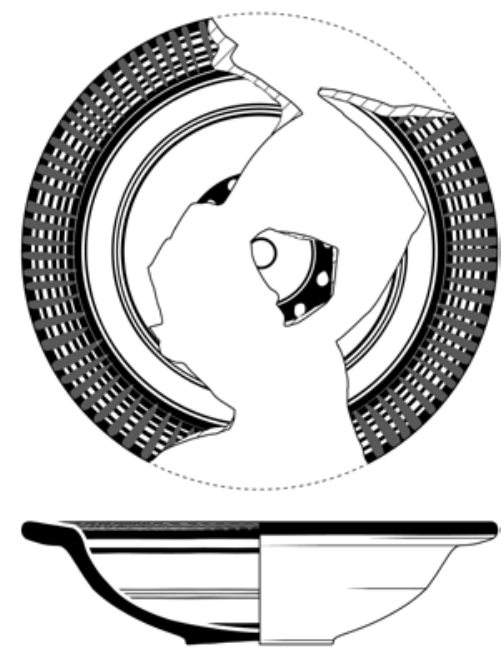

23

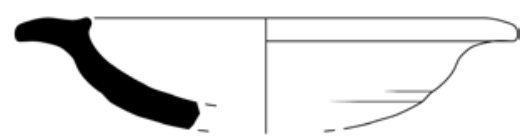

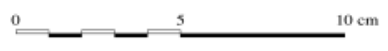

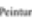

[ Noin

- Rouper
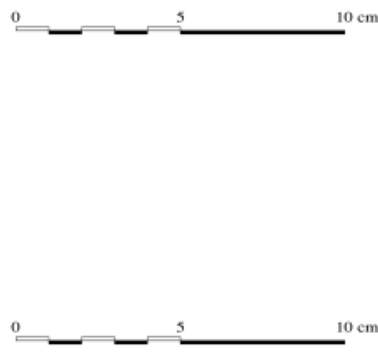

24

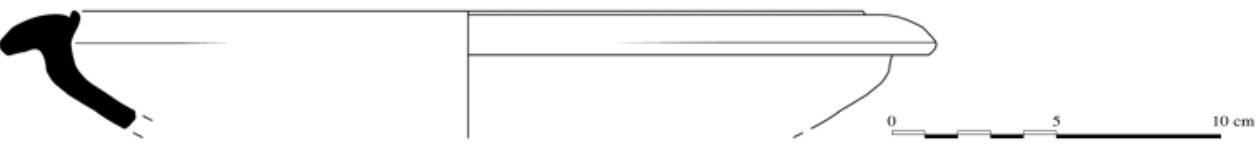

Fig. 21. - d/b.pw.4 (dessin EFA, J. Humbert).

Fig. 22. - d/b.bichr.8 (dessin EFA, J. Humbert).

Fig. 23. - d/b.rs.4 (dessin EFA, J. Humbert).

Fig. 24. - d/b.bs.2 (dessin EFA, J. Humbert). 
25
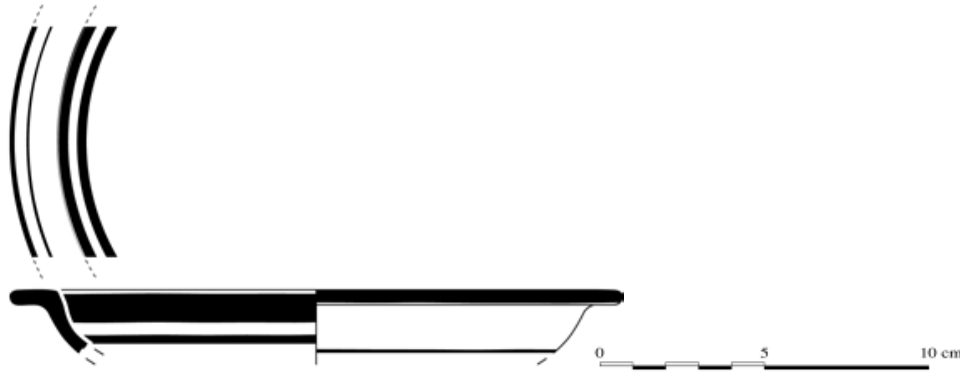

26
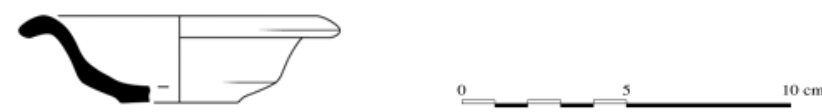

27
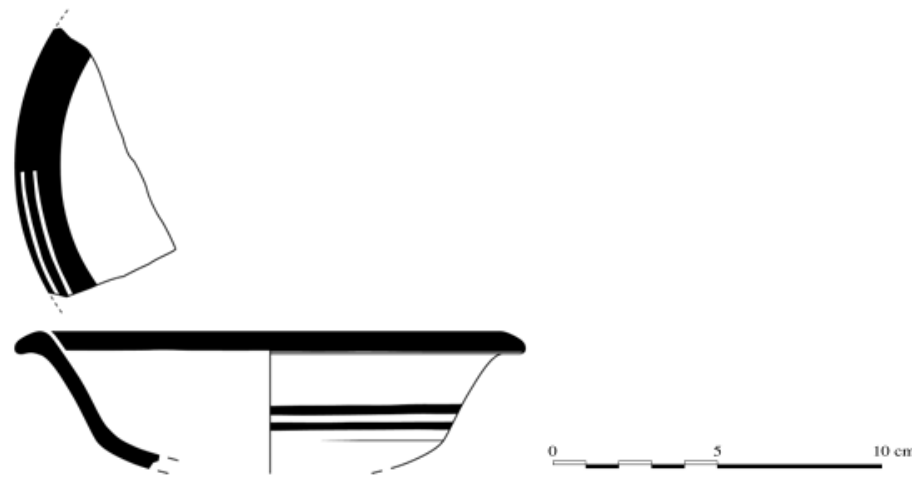

28
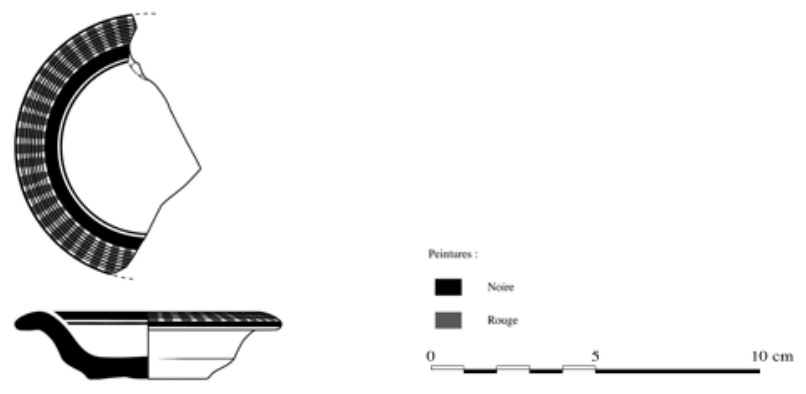

Fig. 25. - d/b.bor.3 (dessin EFA, J. Humbert).

Fig. 26. - d/b.pw.5 (dessin EFA, J. Humbert).

Fig. 27. - d/b.wp.13 (dessin EFA, J. Humbert).

Fig. 28. — d/b.bichr.23 (dessin EFA, J. Humbert). 
29

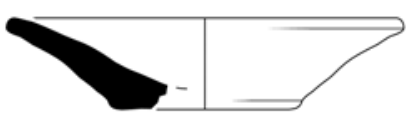

30

31
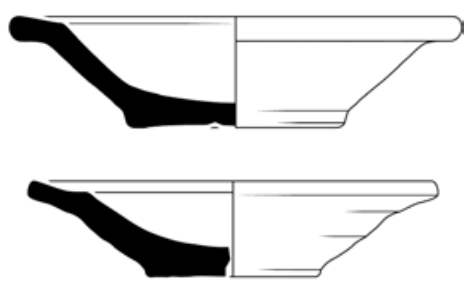
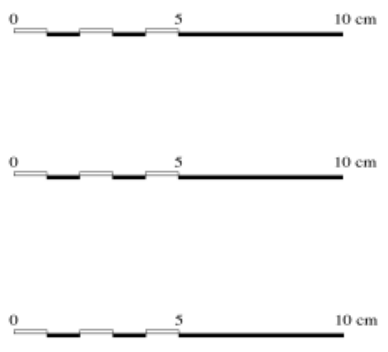

32
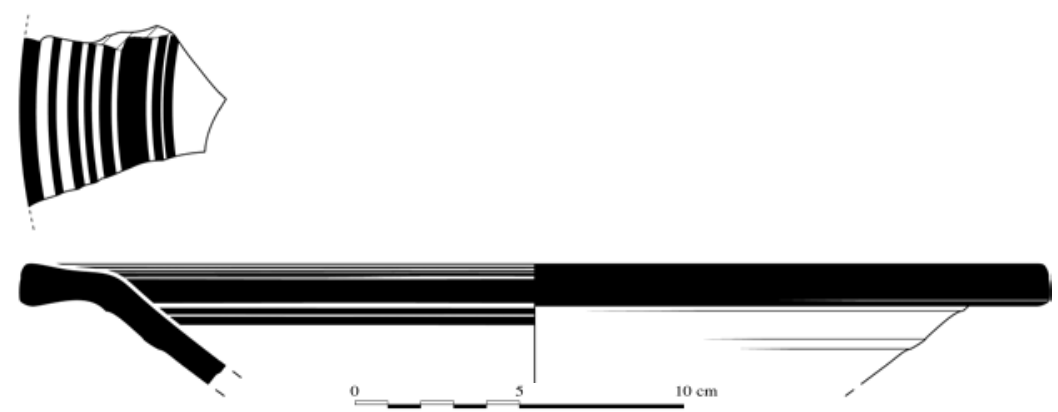

33 a

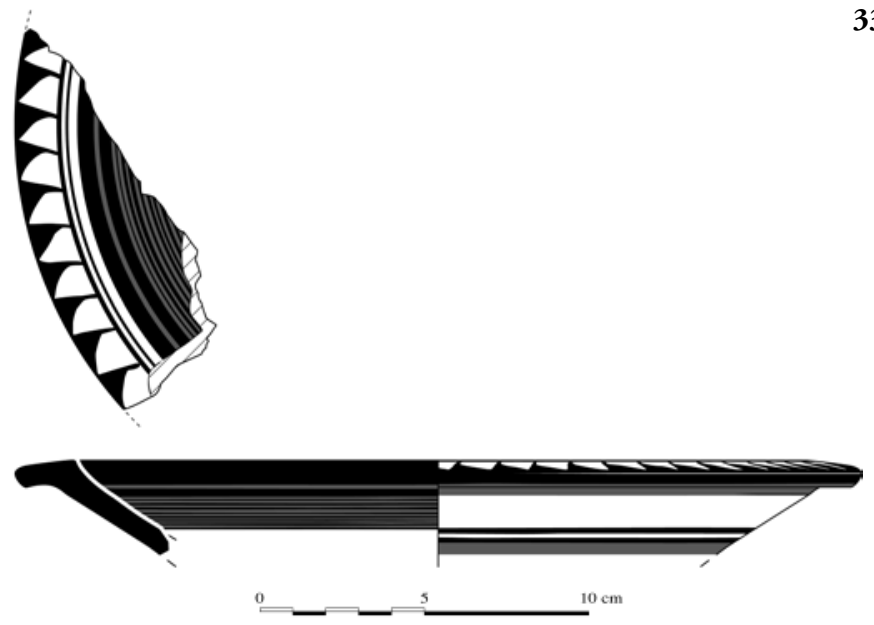

33 b

Fig. 29. - d/b.pw.14 (dessin EFA, J. Humbert).

Fig. 30. - d/b.pw.19 (dessin EFA, J. Humbert).

Fig. 31. - d/b.pw.21 (dessin EFA, J. Humbert).

Fig. 32. - d/b.wp.10 (dessin EFA, J. Humbert).

Fig. 33. - d/b.bichr.12. a. (dessin EFA, J. Humbert), b. (cl. EFA, Ph. Collet). 
34
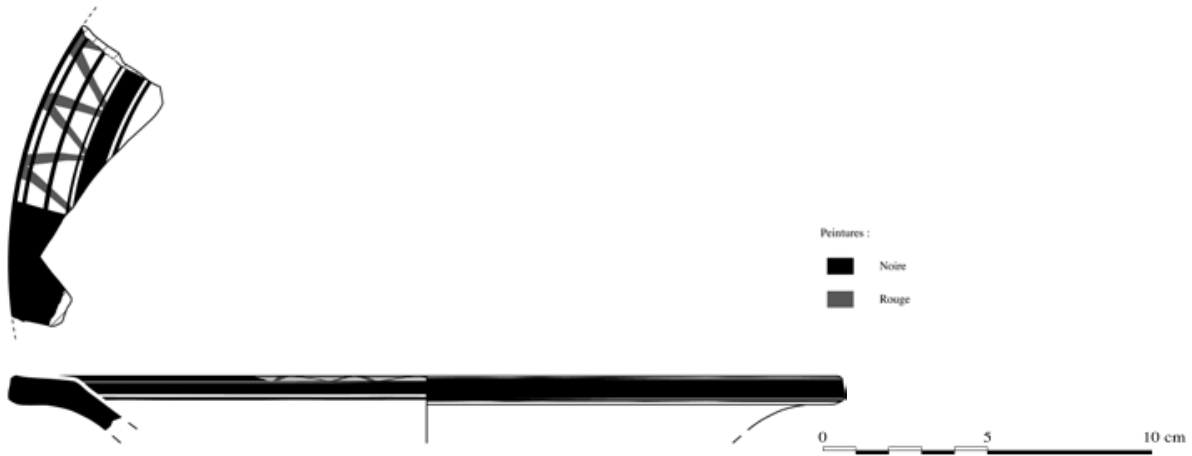

35
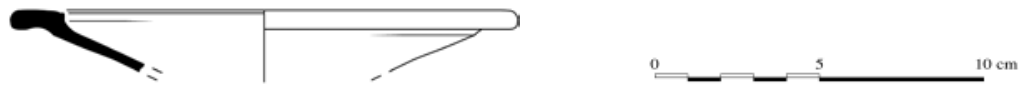

36
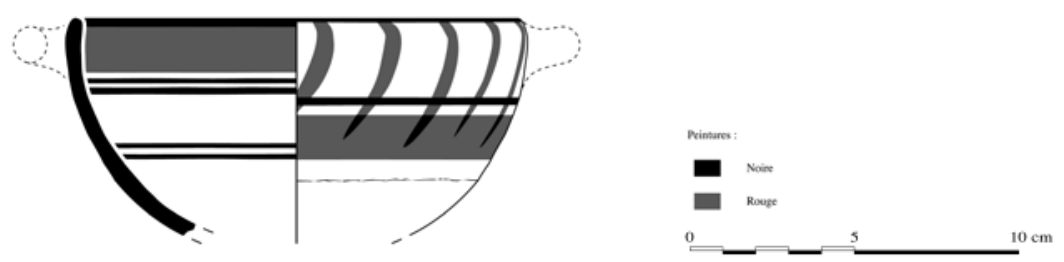

37
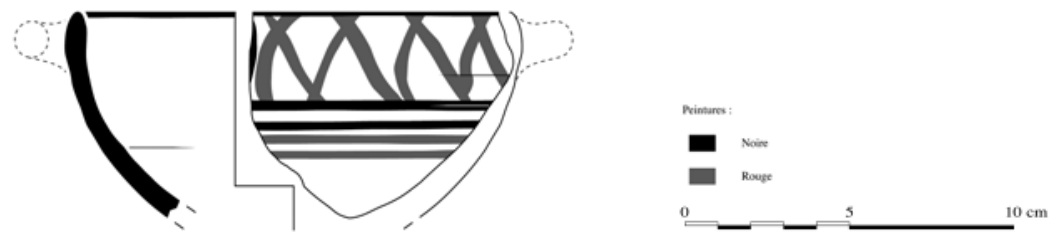

Fig. 34. - d/b.bichr.14 (dessin EFA, J. Humbert).

Fig. 35. — d/b.rs.5 (dessin EFA, J. Humbert).

Fig. 36. — d/b.bichr.17 (dessin EFA, J. Humbert).

Fig. 37. — d/b.bichr.19 (dessin EFA, J. Humbert). 
38

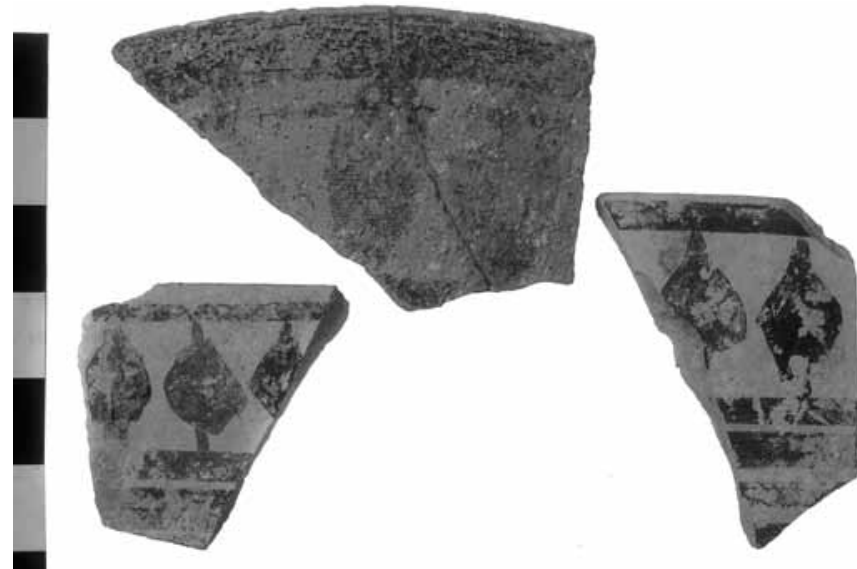

39
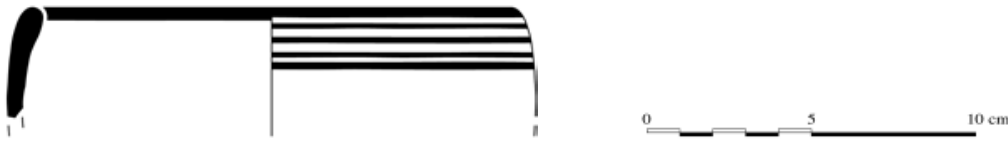

40
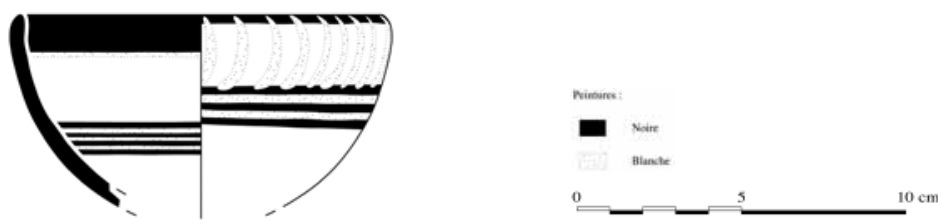

41
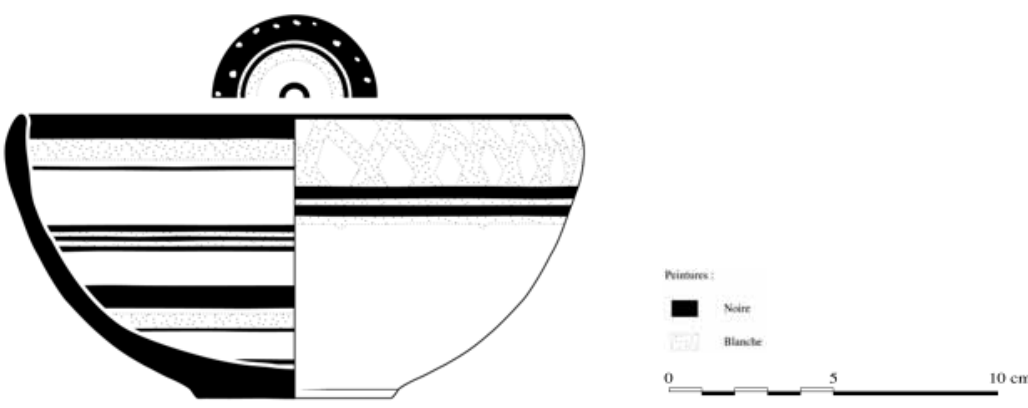

Fig. 38. - Frises de feuilles du « style d'Amathonte» sur des bols Bichr. de type B.2.1 (cl. EFA, Ph. Collet).

Fig. 39. - d/b.bor.5 (dessin EFA, J. Humbert).

Fig. 40. — d/b.bichr.r.11 (dessin EFA, J. Humbert).

Fig. 41. - d/b.bs.7 (dessin EFA, J. Humbert). 
42
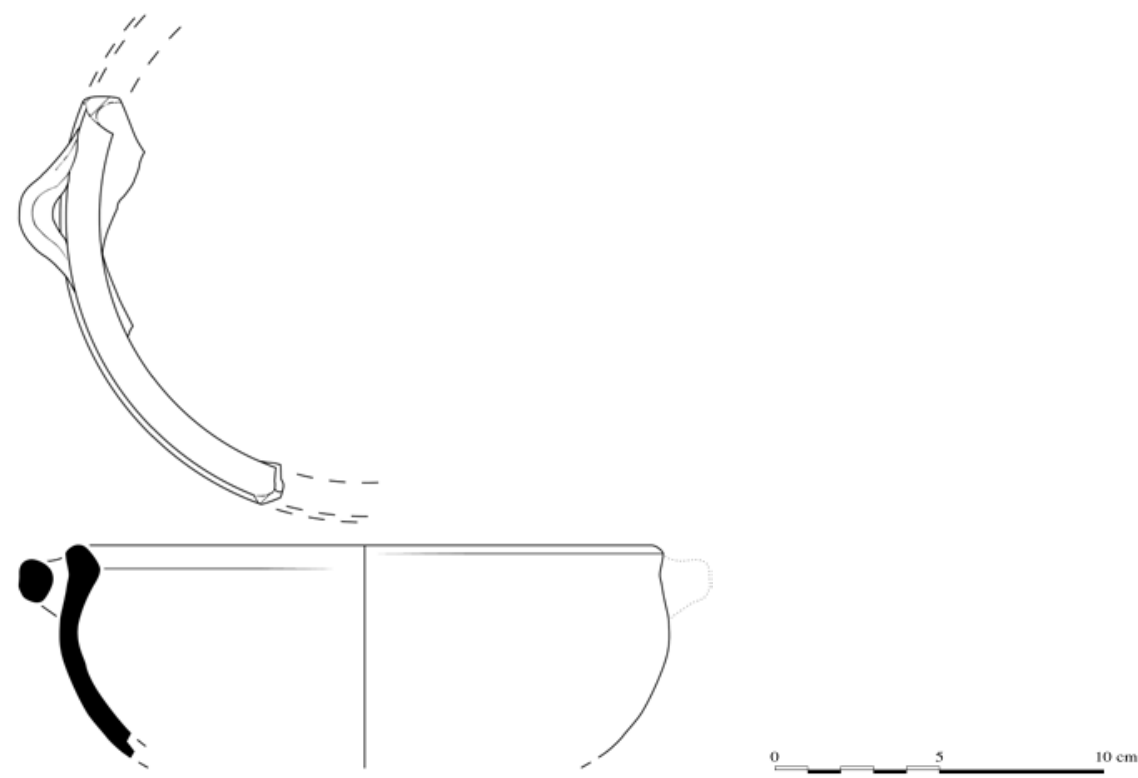

43
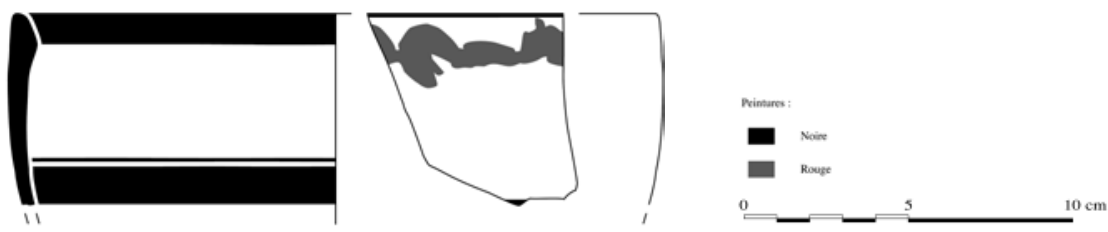

44
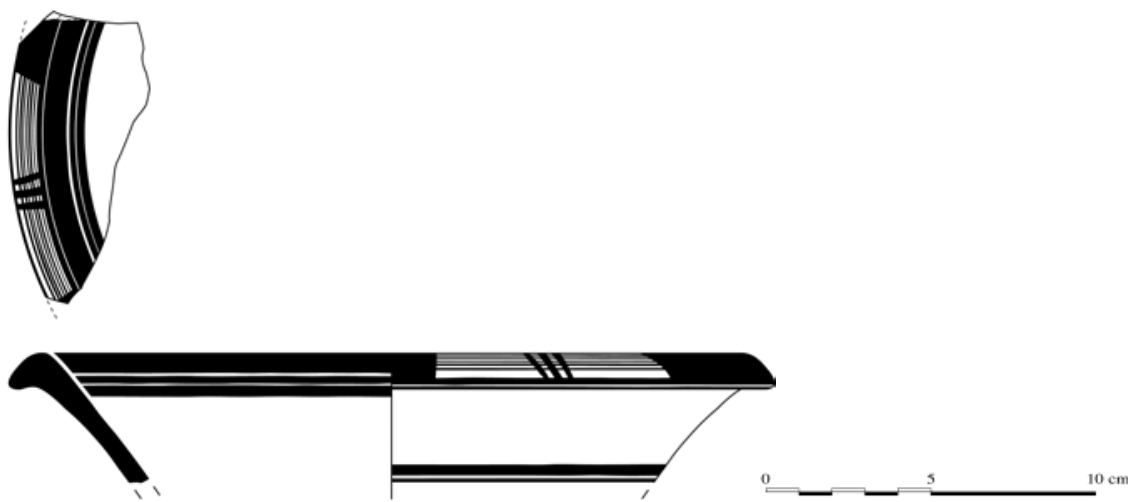

Fig. 42. - d/b.pw.18 (dessin EFA, J. Humbert).

Fig. 43. - d/b.bichr.20 (dessin EFA, J. Humbert).

Fig. 44. — d/b.wp.8 (dessin EFA, J. Humbert).

BCH 133 (2009) 
45

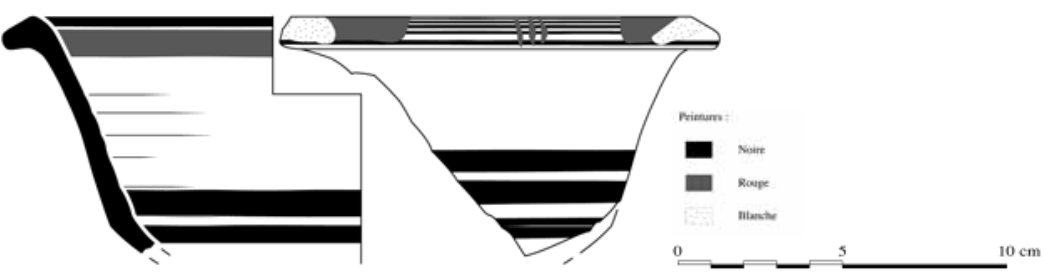

$46 a$
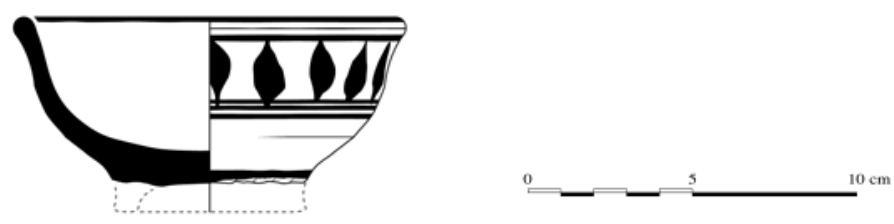

$46 \mathrm{~b}$

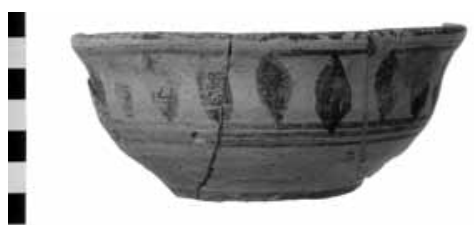

47
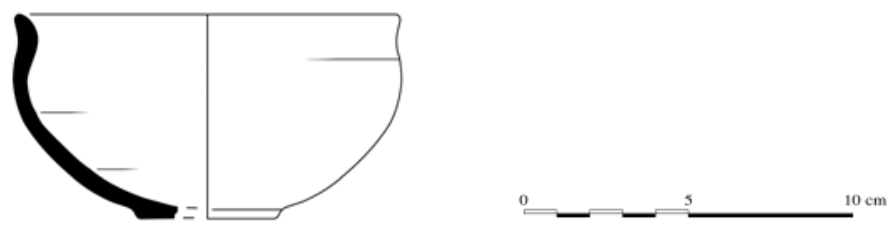

48
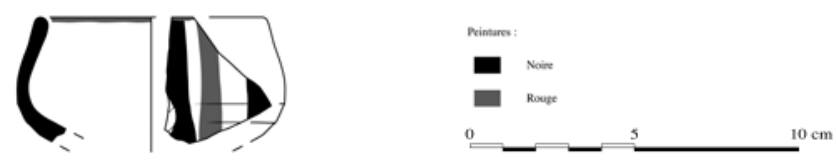

Fig. 45. - d/b.bichr.10 (dessin EFA, J. Humbert).

Fig. 46. - d/b.wp.15. a. (dessin EFA, J. Humbert), b. (cl. EFA, Ph. Collet).

Fig. 47. - d/b.rs.6 (dessin EFA, J. Humbert).

Fig. 48. — d/b.bichr.25 (dessin EFA, J. Humbert). 
49

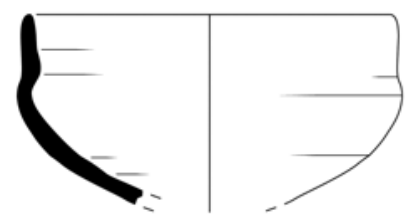

50
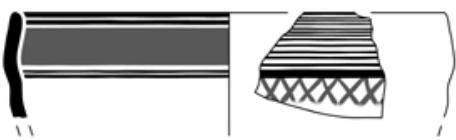

51

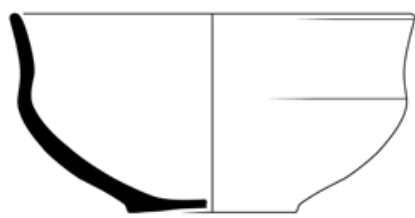

52

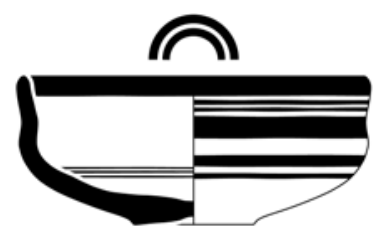

53

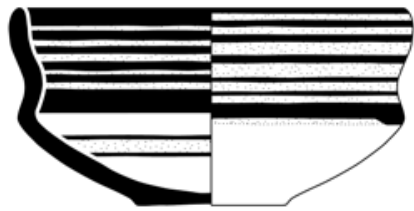

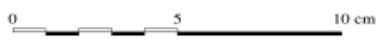
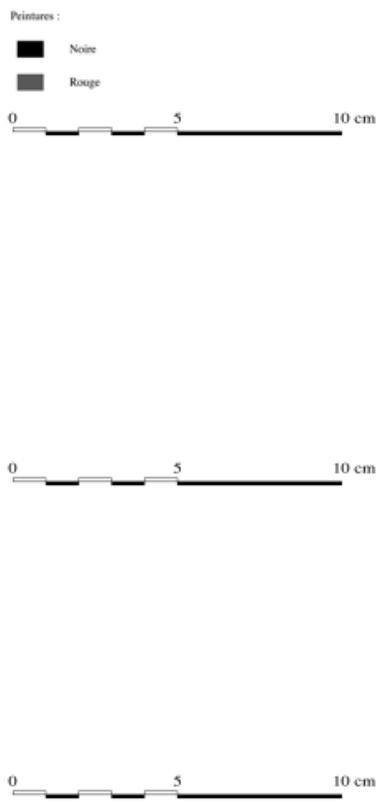

Wimsore

D. Narm

Blunte

${ }^{0}-5^{5}{ }^{10} \mathrm{~cm}$

Fig. 49. — d/b.pw.1 (dessin EFA, J. Humbert).

Fig. 50. — d/b.bichr.26 (dessin EFA, J. Humbert).

Fig. 51. — d/b.rs.7 (dessin EFA, J. Humbert).

Fig. 52. - d/b.bor.7 (dessin EFA, J. Humbert).

Fig. 53. — d/b.bichr.r.10 (dessin EFA, J. Humbert). 
54
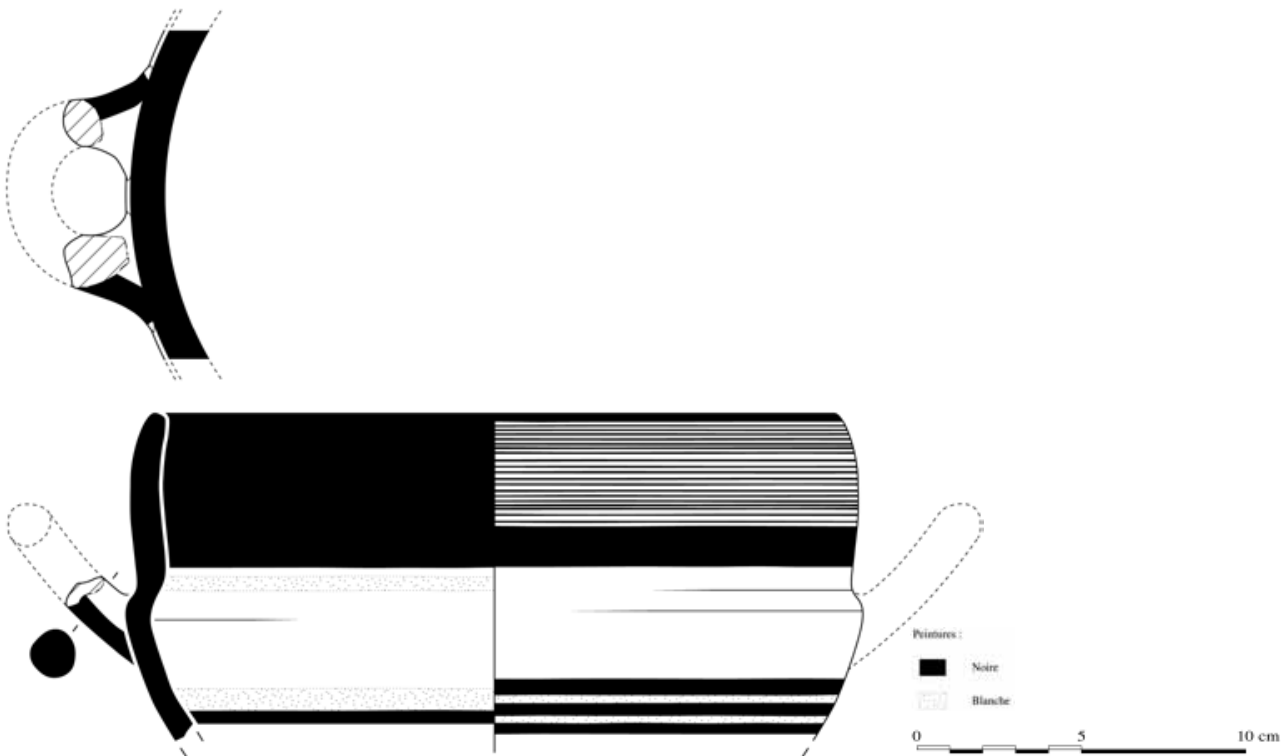

55

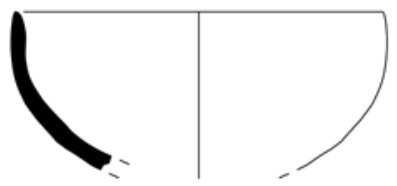
$10 \mathrm{~cm}$

56
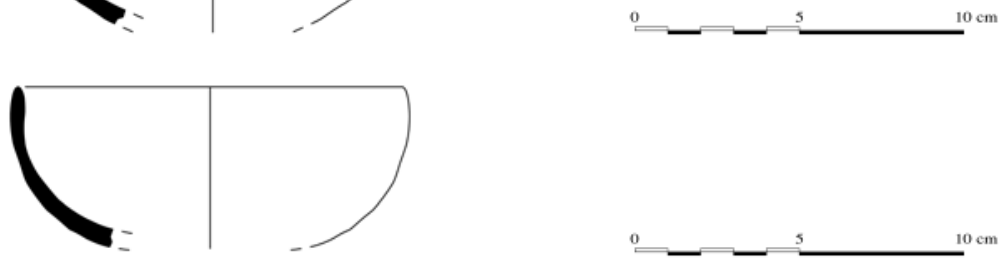

57
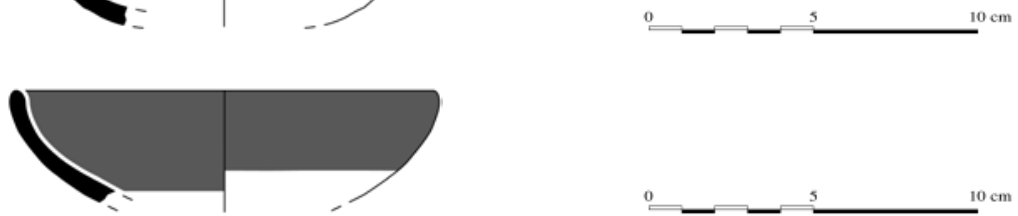

58
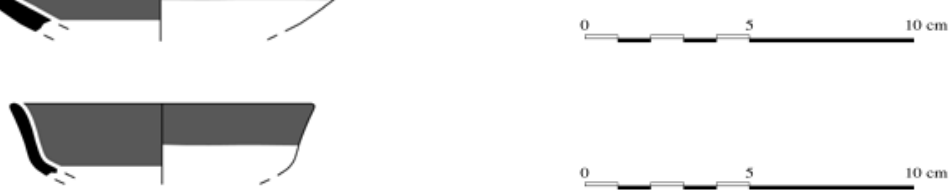

Fig. 54. — d/b.bichr.r.9 (dessin EFA, J. Humbert).

Fig. 55. - d/b.pw.6 (dessin EFA, J. Humbert).

Fig. 56. - d/b.pw.7 (dessin EFA, J. Humbert).

Fig. 57. — d/b.bichr.27 (dessin EFA, J. Humbert).

Fig. 58. — d/b.bichr.28 (dessin EFA, J. Humbert). 
59 a
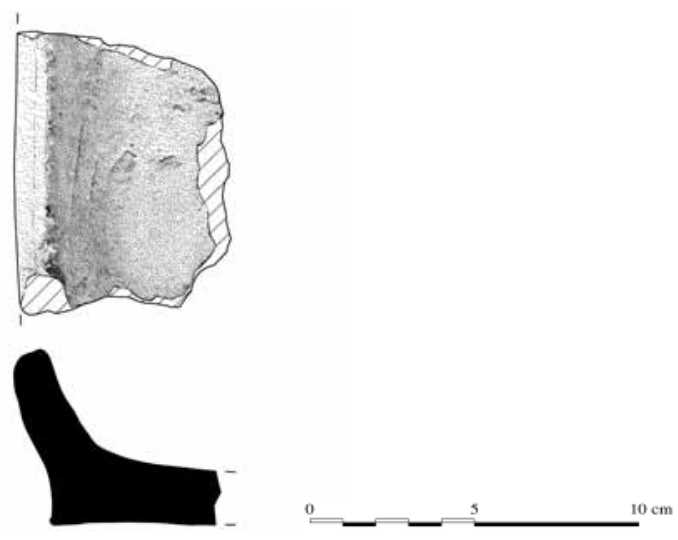

$59 \mathrm{~b}$

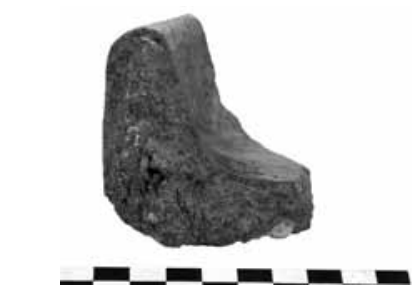

$59 \mathrm{c}$

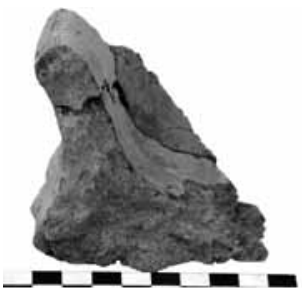

60
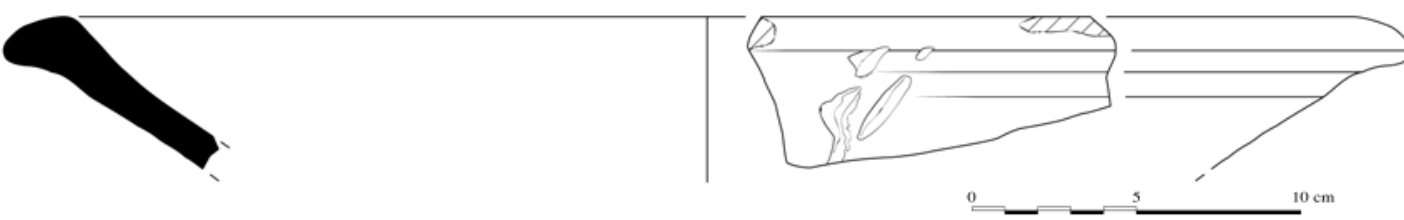

61
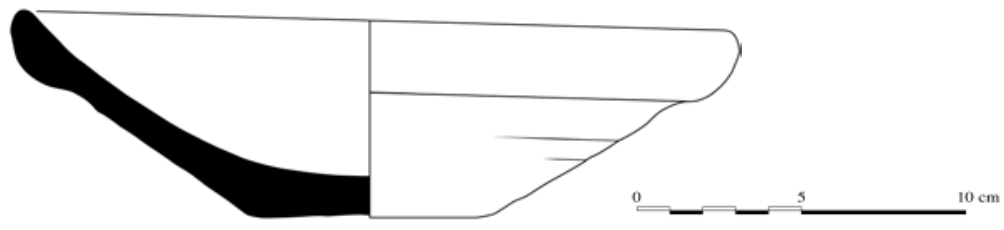

62
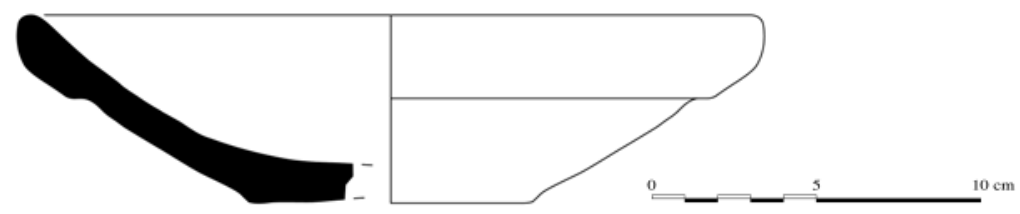

Fig. 59. - d/b.pw.15. a., b. et c. Fragments de soufflets (?) provenant d'un atelier métallurgique, sanctuaire archaïque de Kition-Bamboula (cl. Mission française de Kition et Salamine).

Fig. 60. - d/b.pw.16 (dessin EFA, J. Humbert).

Fig. 61. — d/b.pw.27 (dessin EFA, J. Humbert).

Fig. 62. - d/b.pw.28 (dessin EFA, J. Humbert).

BCH 133 (2009) 
63

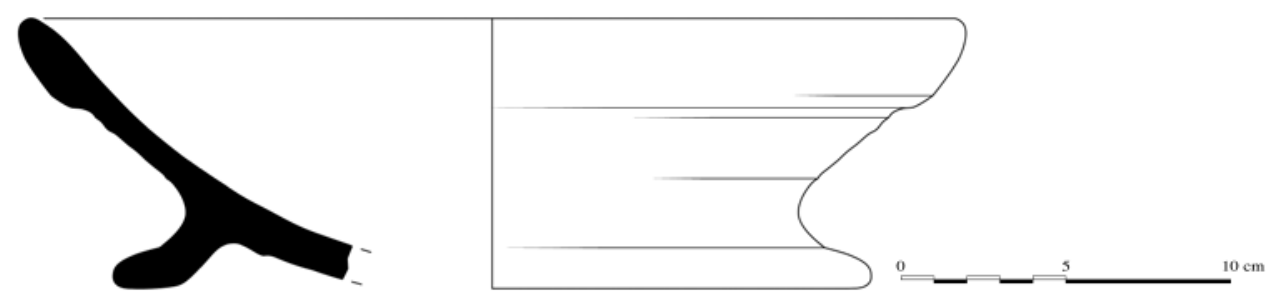

64

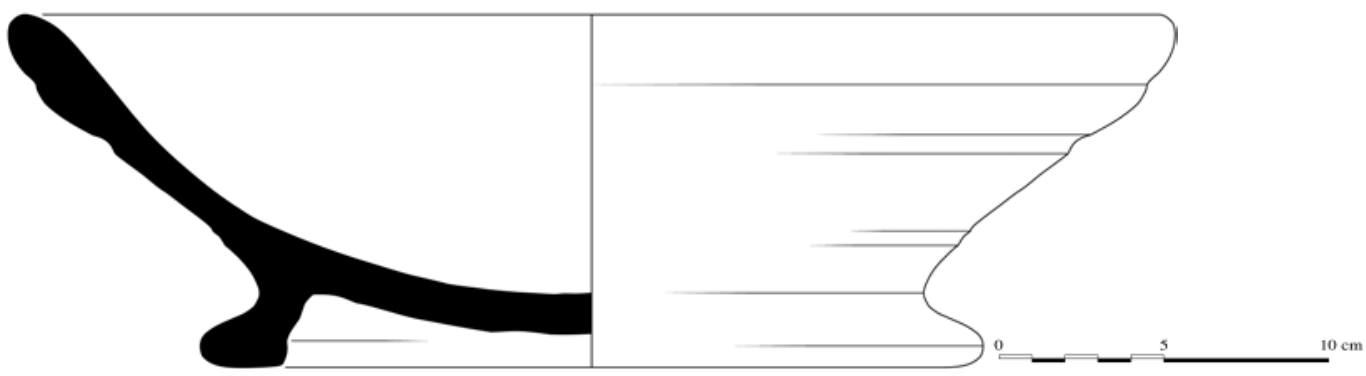

65
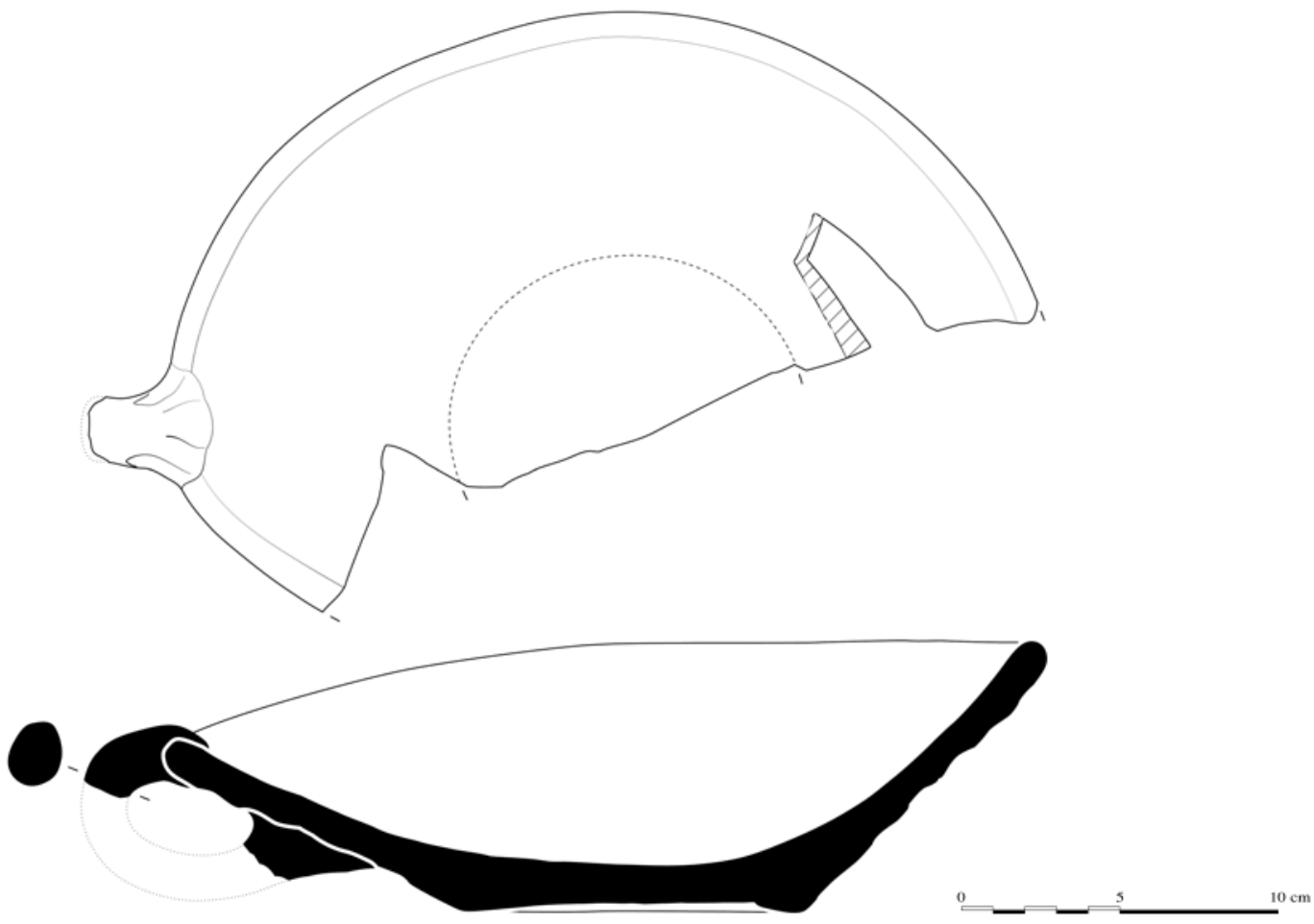

Fig. 63. - d/b.pw.31 (dessin EFA, J. Humbert).

Fig. 64. - d/b.pw.32 (dessin EFA, J. Humbert).

Fig. 65. - d/b.pw.33 (dessin EFA, J. Humbert). 
66
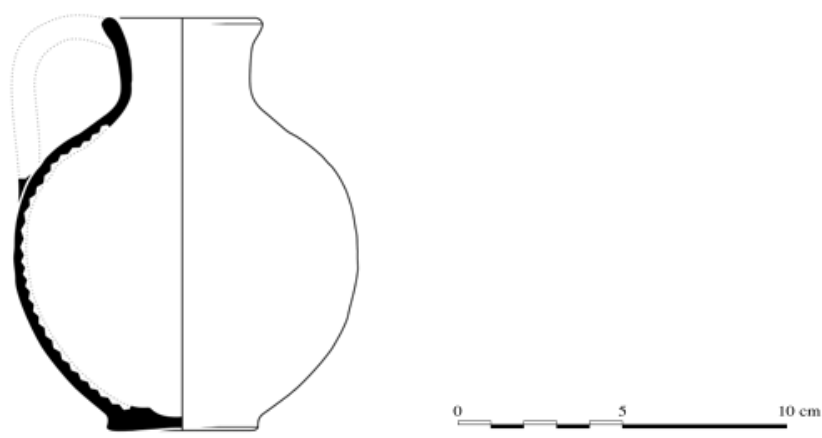

67

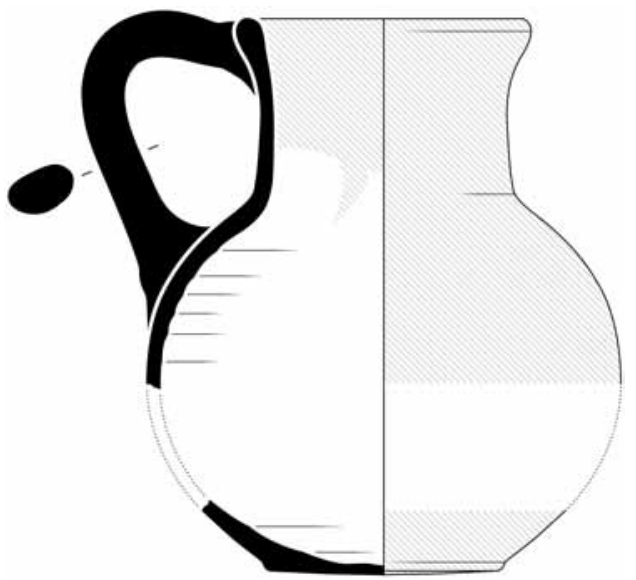

Engobe

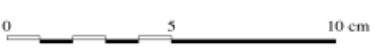

68
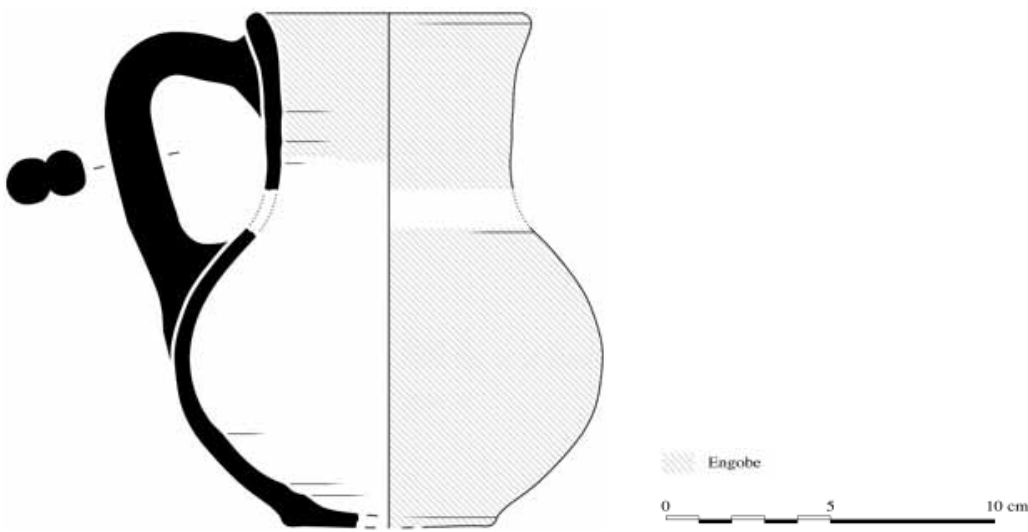

Fig. 66. $-\mathrm{d} /$ cr.pw.1 (dessin EFA, J. Humbert).

Fig. 67. — d/cr.rs.9 (dessin EFA, J. Humbert).

Fig. 68. — d/cr.rs.6 (dessin EFA, J. Humbert).

BCH 133 (2009) 
69
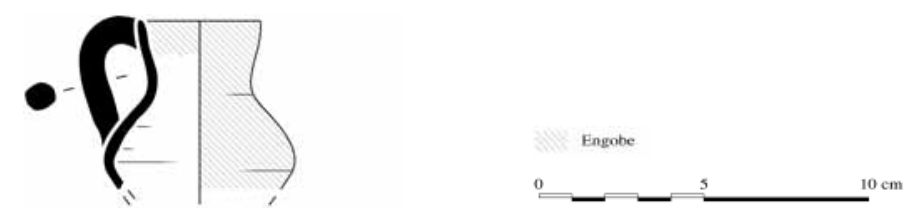

70

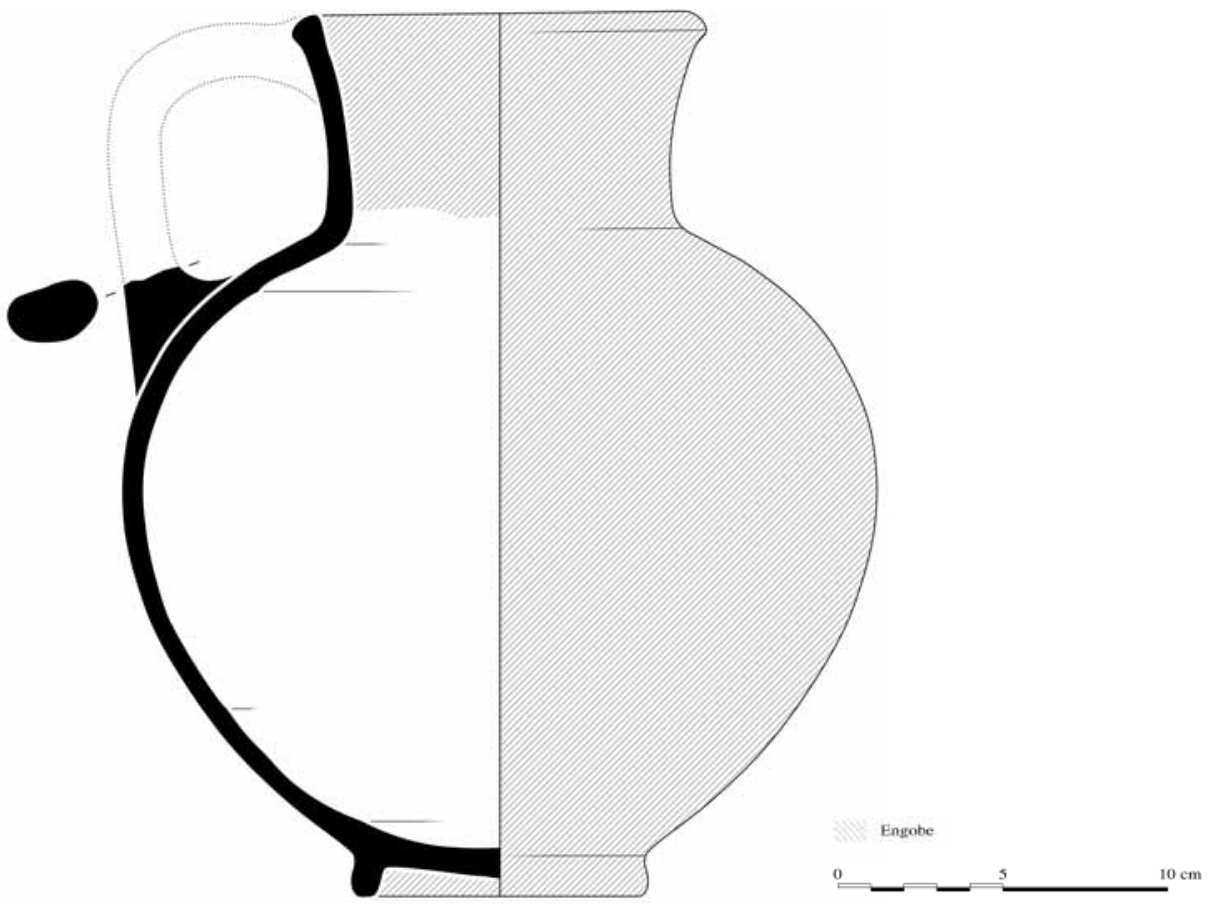

71
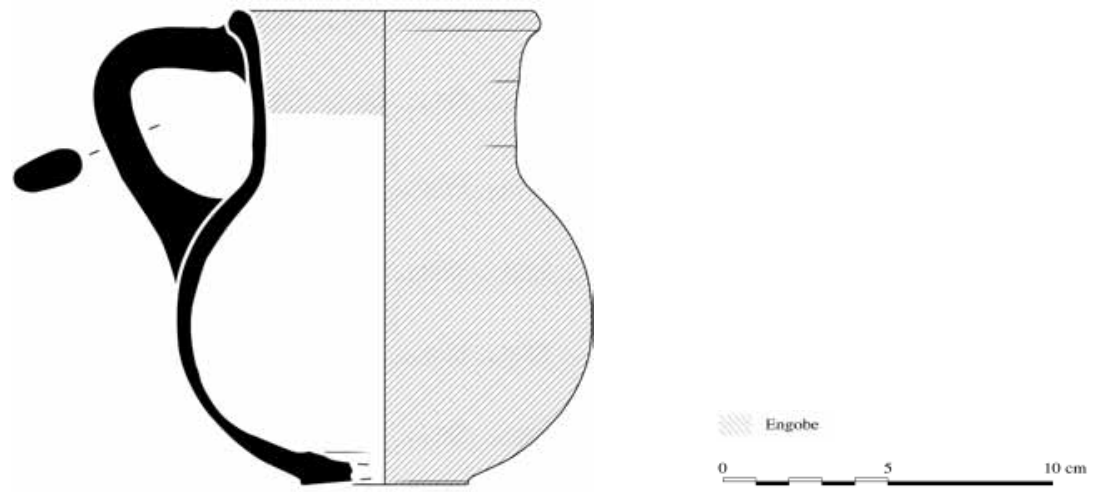

Fig. 69. - d/cr.rs.3 (dessin EFA, J. Humbert).

Fig. 70. - d/cr.bs.1 (dessin EFA, J. Humbert).

Fig. 71. - d/cr.bs.6 (dessin EFA, J. Humbert). 
72

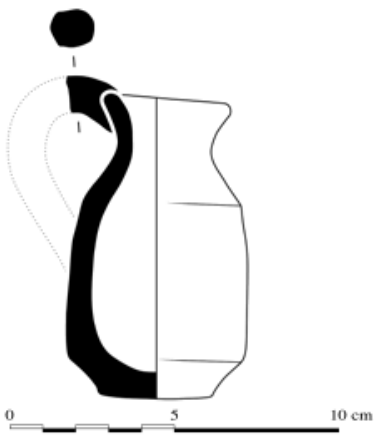

74

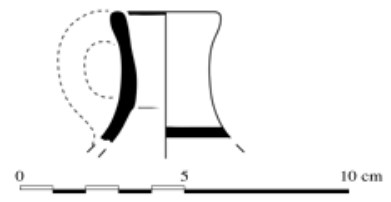

76
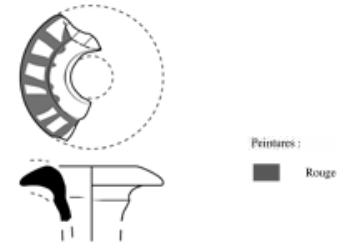

0

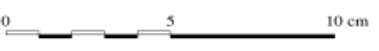

78

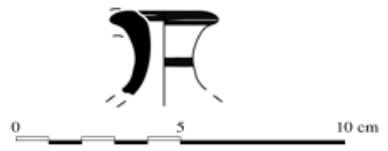

73
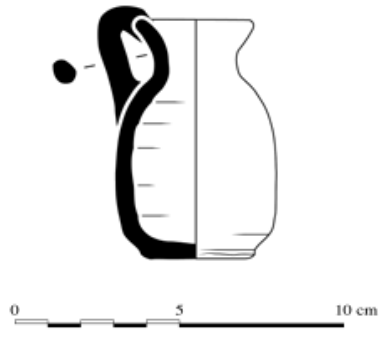

75
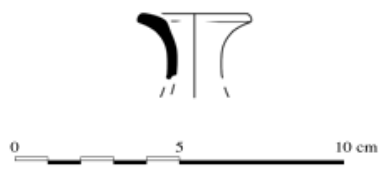

77
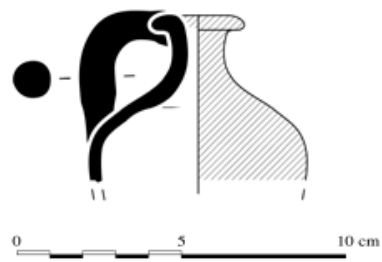

Fig. 72. - AM 2624 (dessin EFA, J. Humbert).

Fig. 73. - AM 2628 (dessin EFA, J. Humbert).

Fig. 74. — d/cr.wp.1 (dessin EFA, J. Humbert).

Fig. 75. - d/cr.pw.5 (dessin EFA, J. Humbert).

Fig. 76. - d/cr.bichr.4 (dessin EFA, J. Humbert).

Fig. 77. — d/cr.bs.3 (dessin EFA, J. Humbert).

Fig. 78. - d/cr.bor.3 (dessin EFA, J. Humbert). 


$$
{ }^{\top}
$$


82

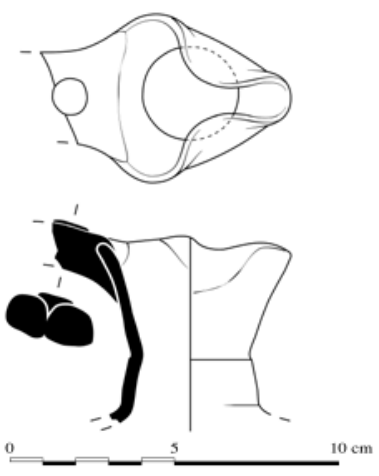

84
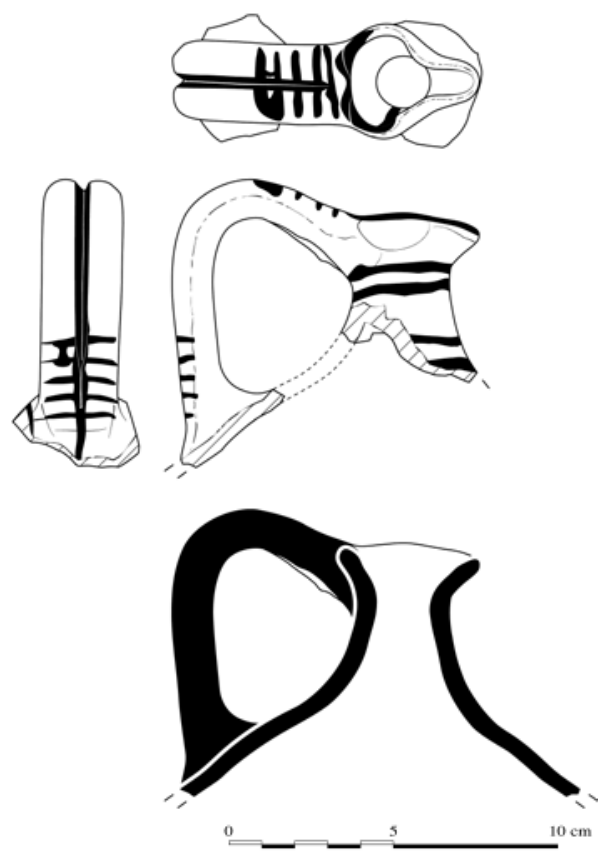

83

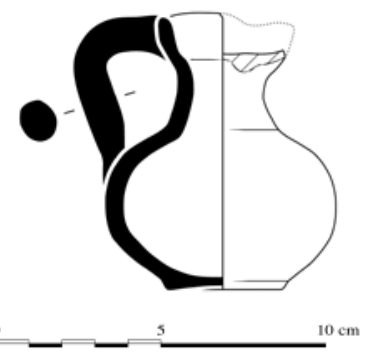

85

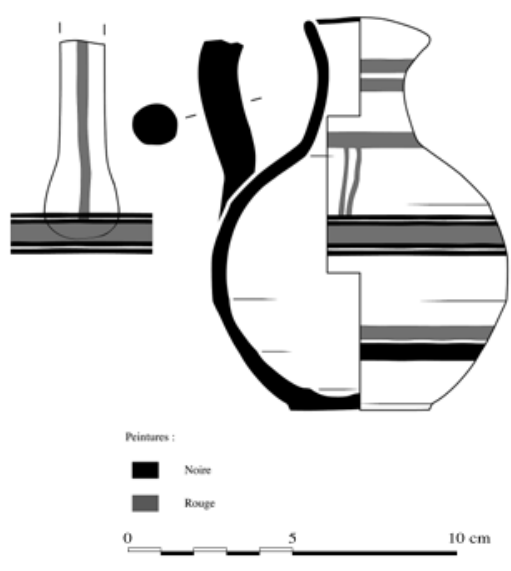

Fig. 82. - d/cr.pw.8 (dessin EFA, J. Humbert).

Fig. 83. - d/cr.pw.9 (dessin EFA, J. Humbert).

Fig. 84. - d/cr.wp.2 (dessin EFA, J. Humbert).

Fig. 85. - d/cr.bichr.5 (dessin EFA, J. Humbert). 
86
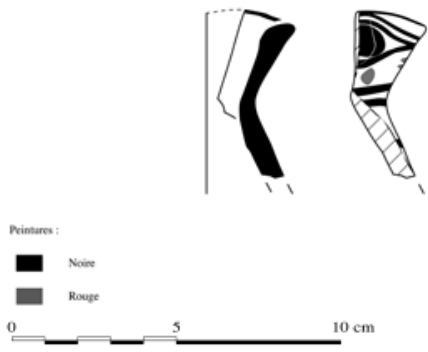

88

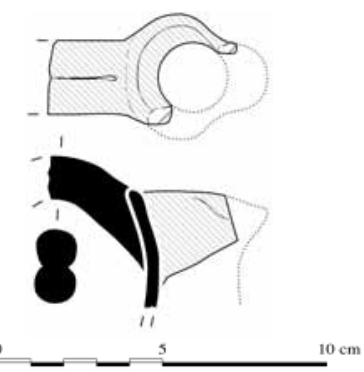

90

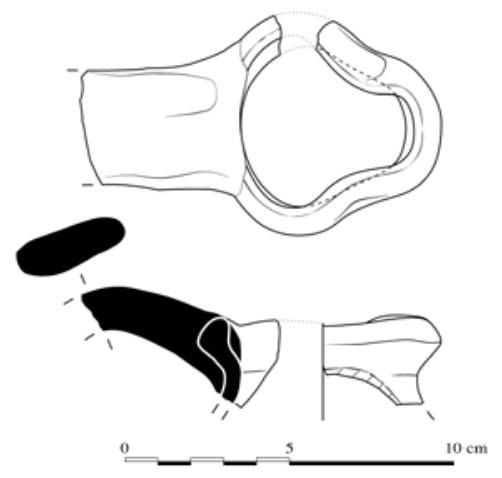

Fig. 86. - d/cr.bichr.7 (dessin EFA, J. Humbert). Fig. 87. — d/cr.bichr.9 (dessin EFA, J. Humbert). Fig. 88. — d/cr.rs.1 (dessin EFA, J. Humbert).

Fig. 99. — $d$ /cr.bor.1 (dessin EFA, J. Humbert).

Fig. 90. - d/cr.pw.11 (dessin EFA, J. Humbert).

Fig. 91. — d/cr.wp.5 (dessin EFA, J. Humbert).
87

89
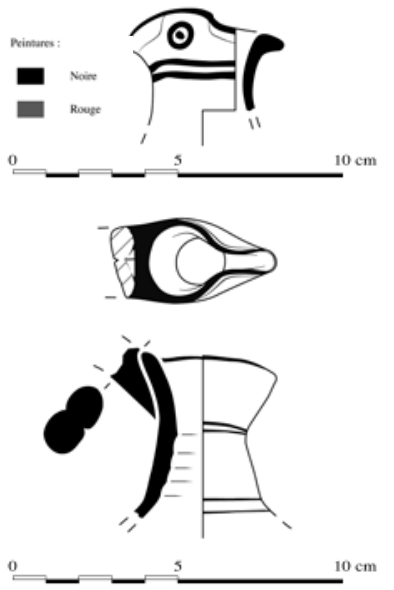

91
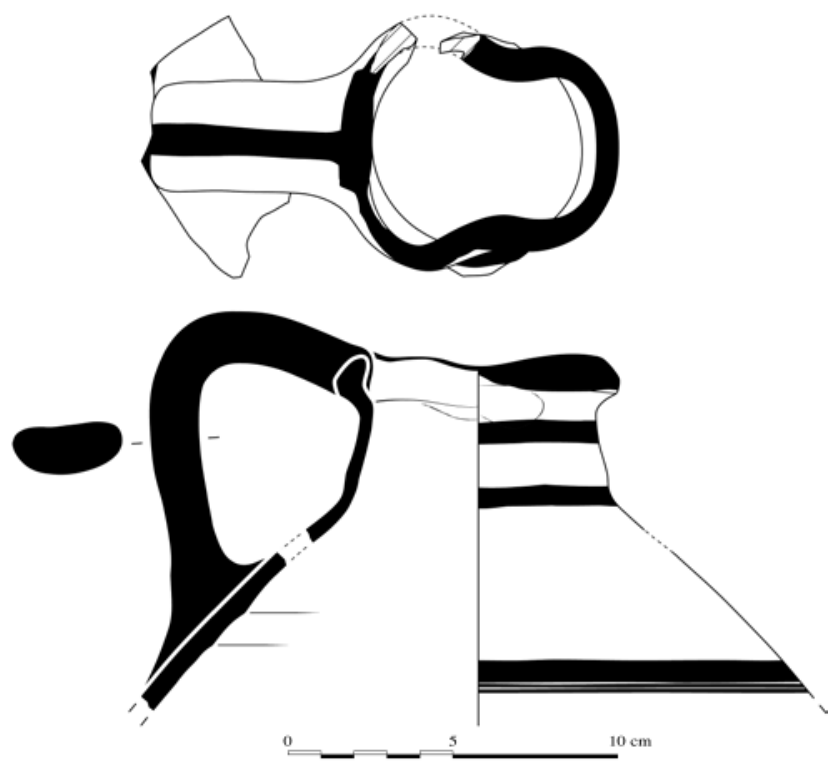
92

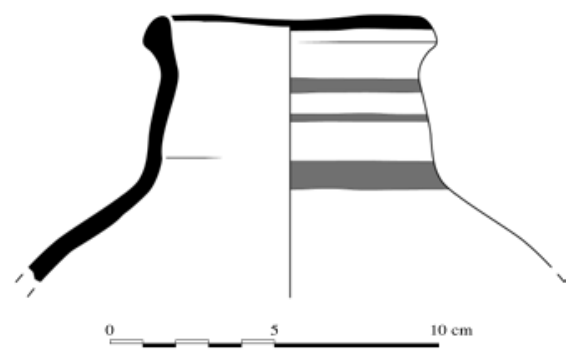

94

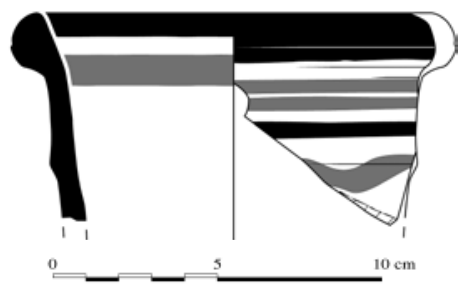

96

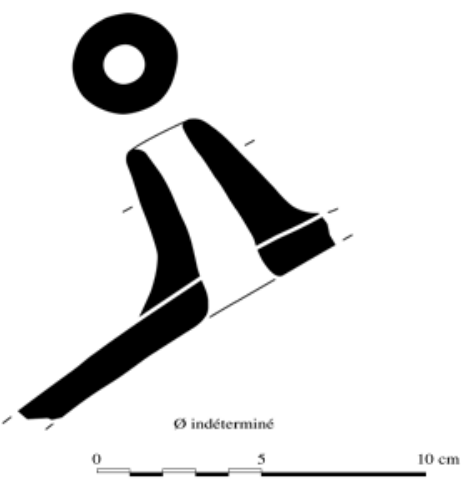

Fig. 92. - d/cr.bichr.8 (dessin EFA, J. Humbert).

Fig. 93. - d/cr.wp.3 (dessin EFA, J. Humbert).

Fig. 94. — d/cr.bichr.2 (dessin EFA, J. Humbert).

Fig. 95. - d/cr.bor.4 (dessin EFA, J. Humbert).

Fig. 96. $-\mathrm{d} / \mathrm{cr}$.pw.7 (dessin EFA, J. Humbert).
93
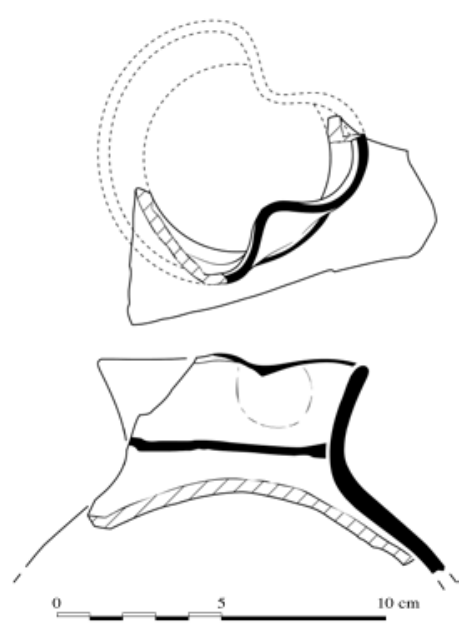

95

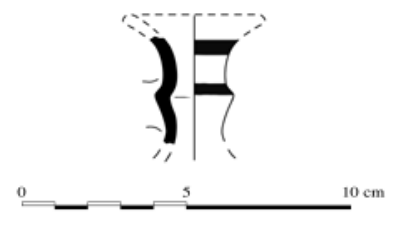

BCH 133 (2009) 
97
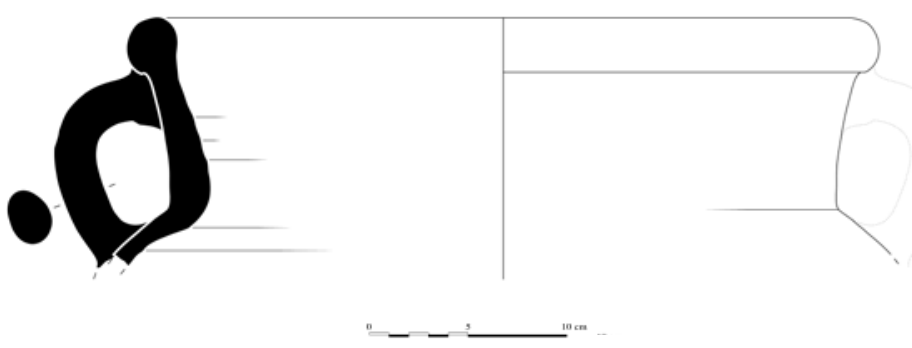

98
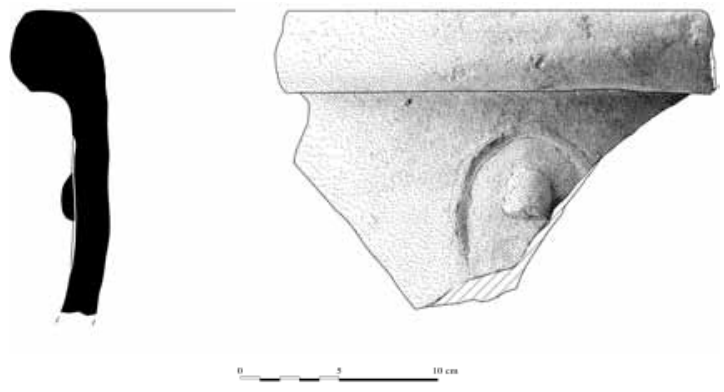

99
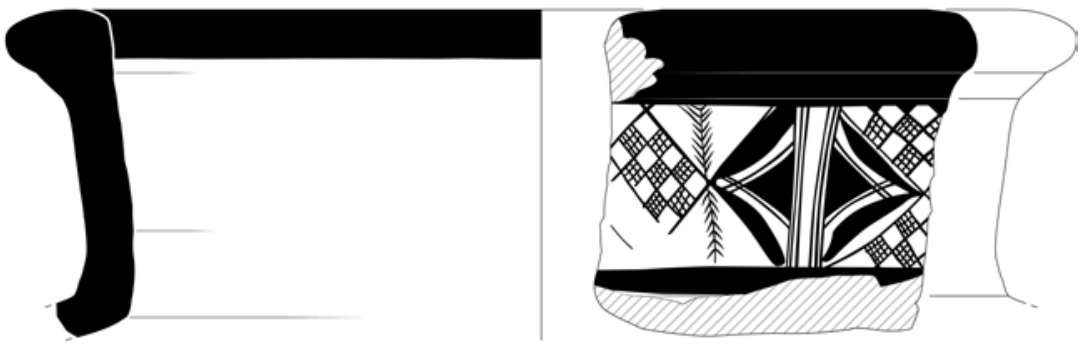

Fig. 97. — d/a.pw.14 (dessin EFA, J. Humbert).

Fig. 98. — d/a.pw.18 (dessin EFA, J. Humbert).

Fig. 99. — d/a.wp.1 (dessin EFA, J. Humbert). 
100
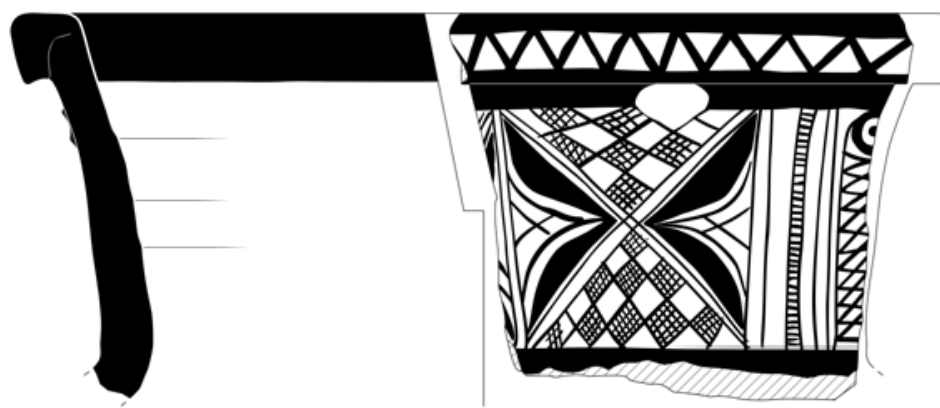

101
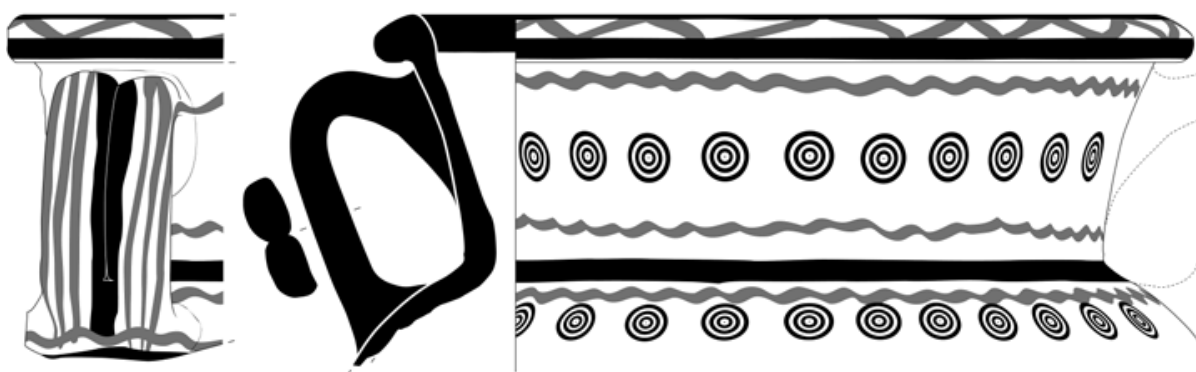

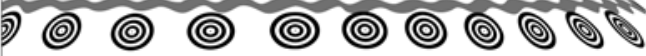

102
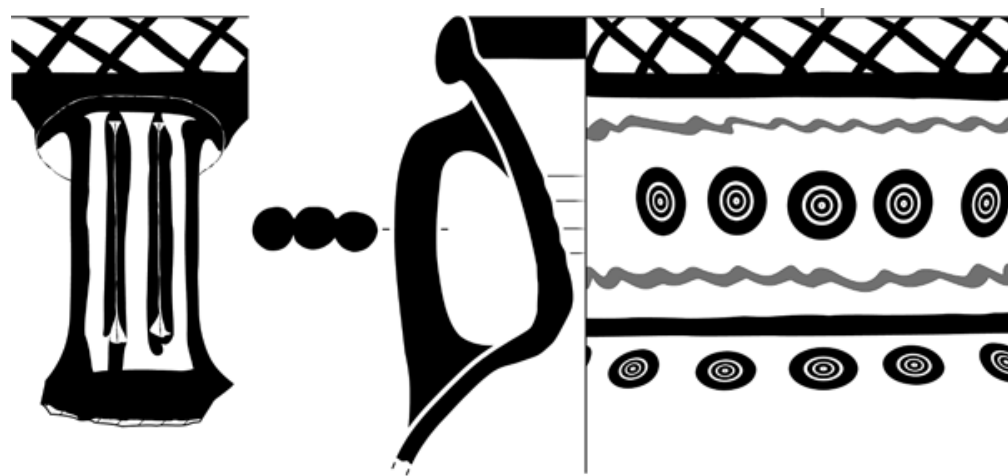

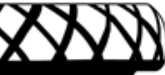

然。

(2)

(2)

(9)

(2)
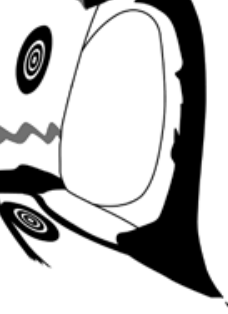

Fig. 100. - d/a.wp.2 (dessin EFA, J. Humbert).

Fig. 101. — d/a.bichr.2 (dessin EFA, J. Humbert).

Fig. 102. — d/a.bichr.1 (dessin EFA, J. Humbert).

BCH 133 (2009) 
103
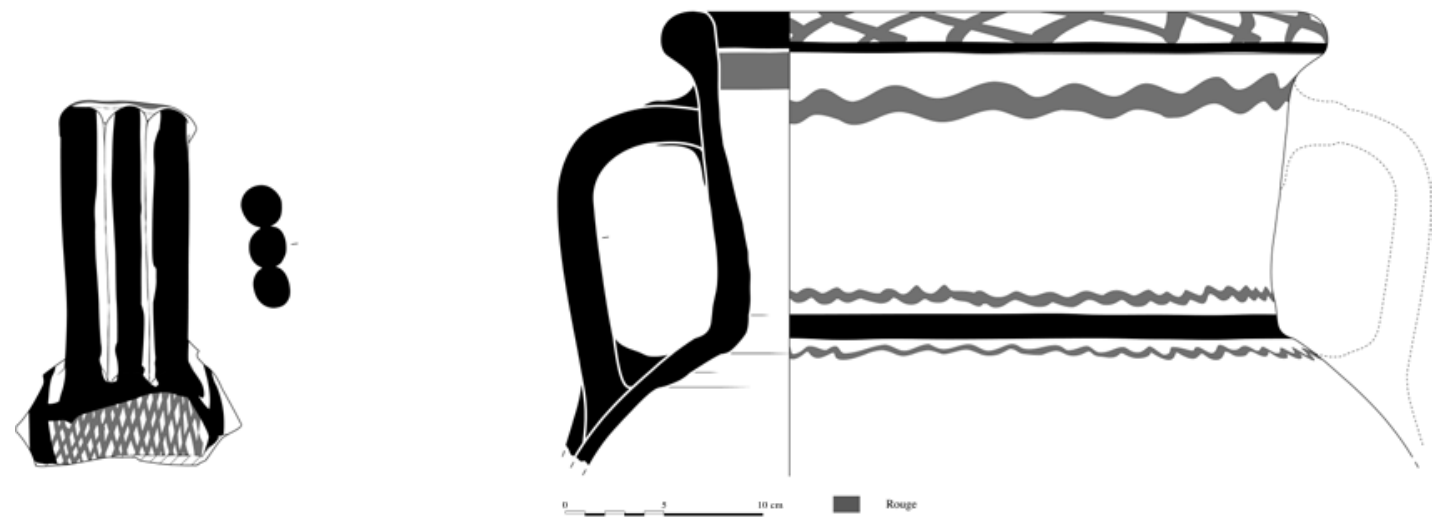

104
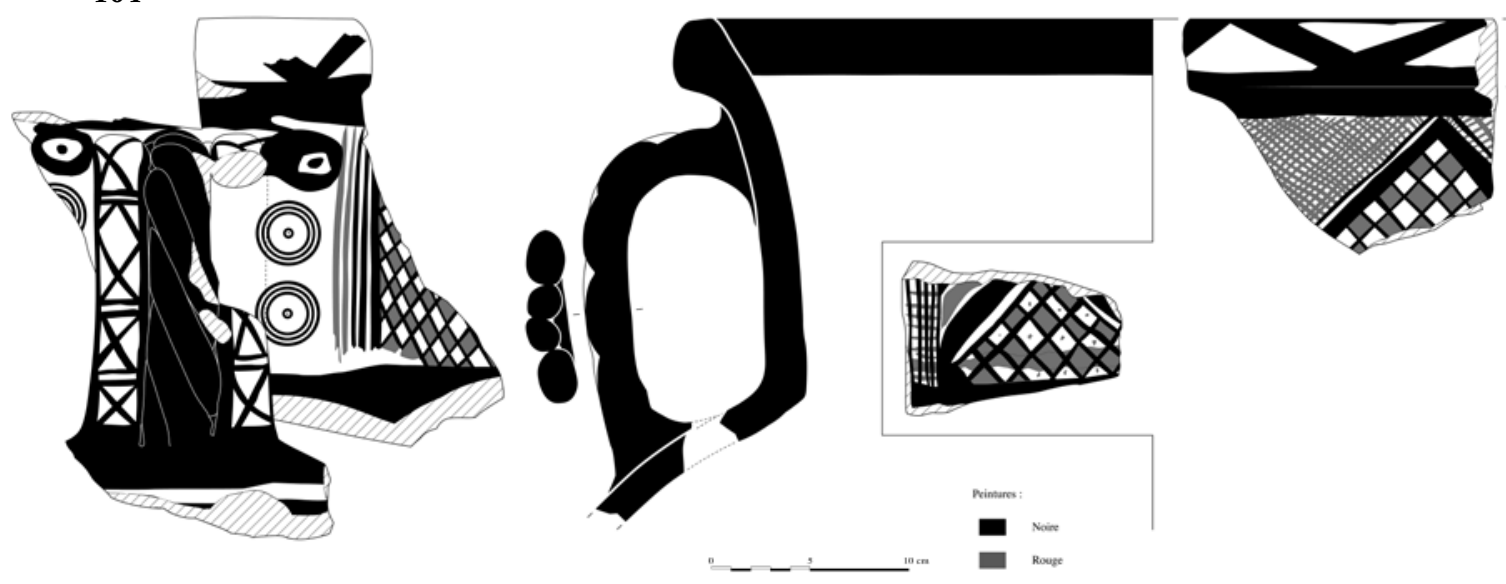

105

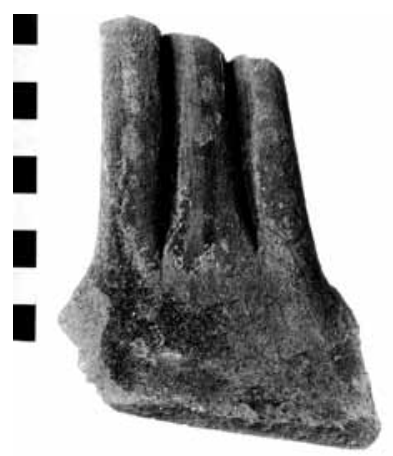

Fig. 103. - d/a.bichr.3 (dessin EFA, J. Humbert).

Fig. 104. — d/a.bichr.4 (dessin EFA, J. Humbert).

Fig. 105. - Anse trifide d'amphore Bichr.R. de type A.1 (cl. EFA, Ph. Collet). 
106

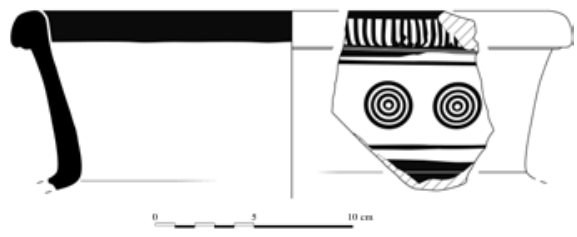

107

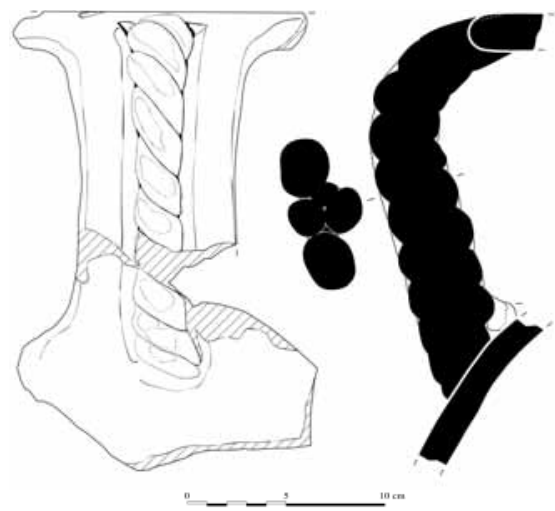

108

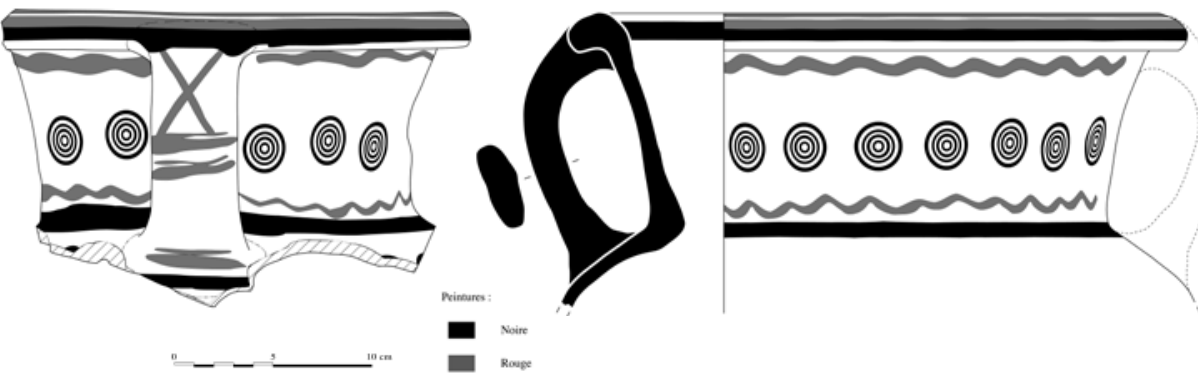

109

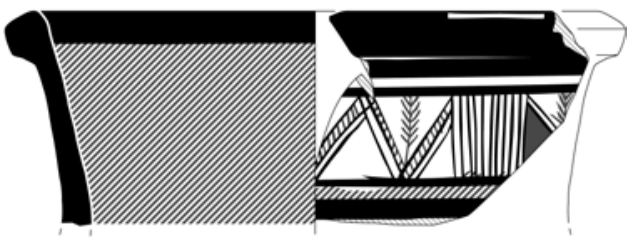

- Peinture noire

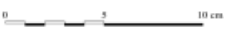

WII Engobe rouge

Fig. 106. — d/a.bor.3 (dessin EFA, J. Humbert).

Fig. 107. — d/a.pw.15 (dessin EFA, J. Humbert).

Fig. 108. — d/a.bichr.5 (dessin EFA, J. Humbert).

Fig. 109. — d/a.bor.2 (dessin EFA, J. Humbert).

BCH 133 (2009) 
110

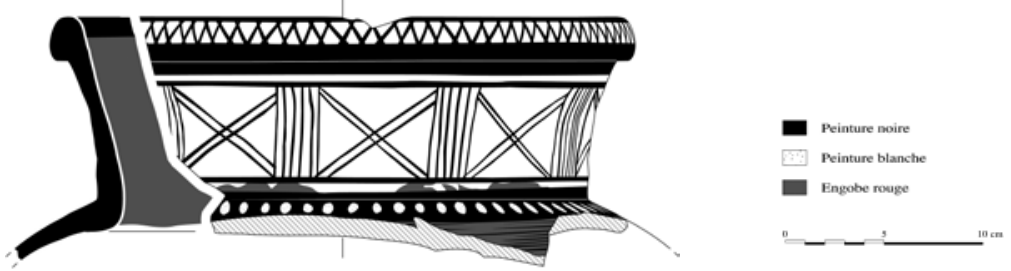

111
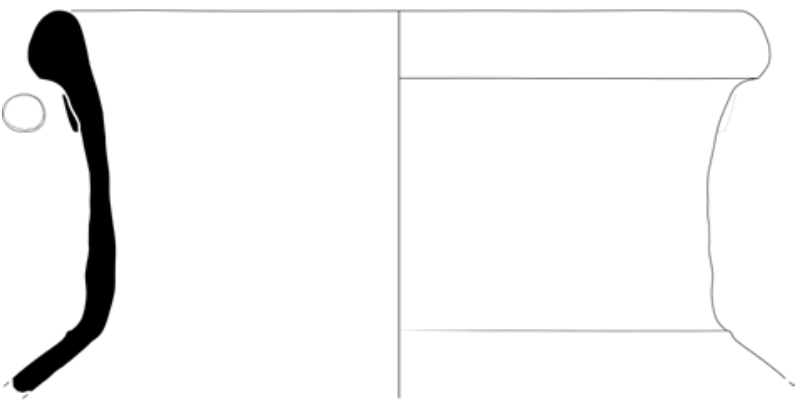

112

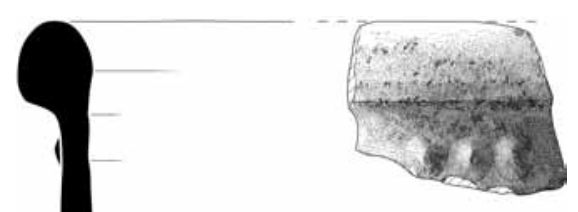

113
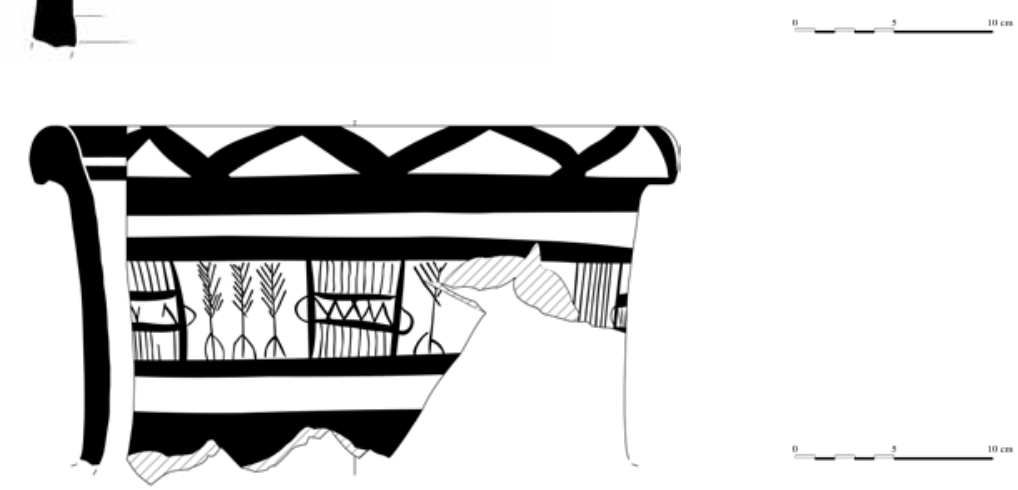

Fig. 110. - d/a.bichr.r.4 (dessin EFA, J. Humbert).

Fig. 111. - d/a.pw.17 (dessin EFA, J. Humbert).

Fig. 112. - d/a.pw.19 (dessin EFA, J. Humbert).

Fig. 113. - d/a.wp.4 (dessin EFA, J. Humbert). 
114

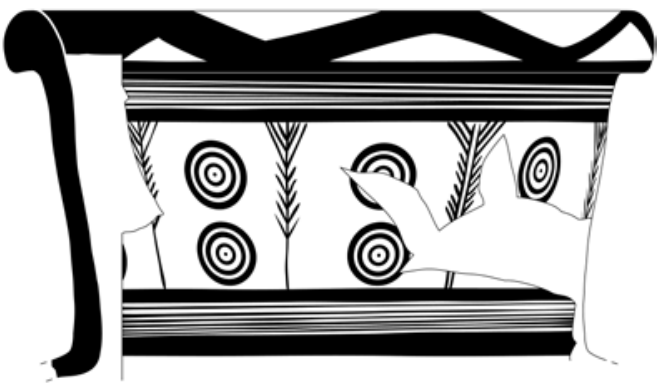

115
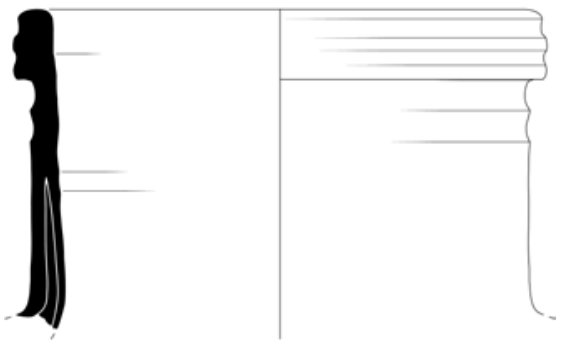

116
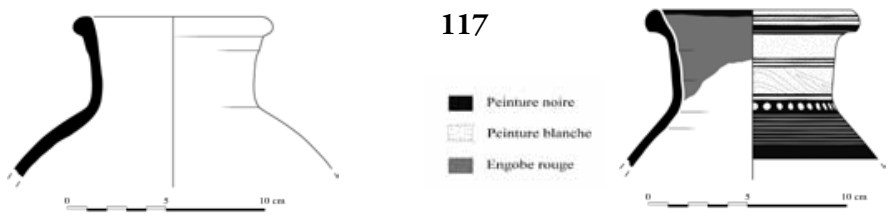

118

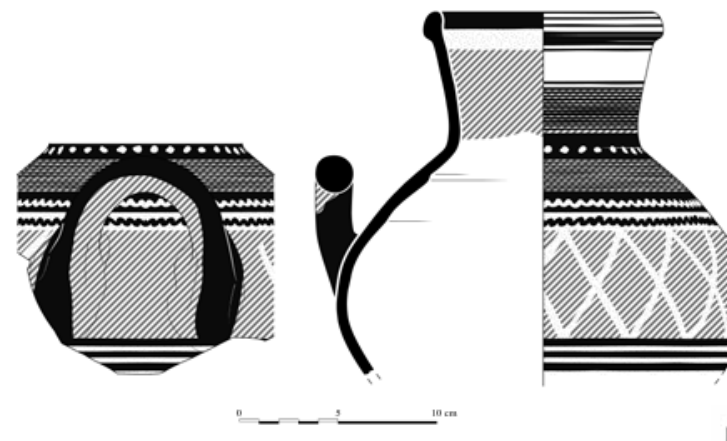

Fig. 114. - d/a.wp.6 (dessin EFA, J. Humbert).

119

Fig. 115. - d/a.pw.20 (dessin EFA, J. Humbert).

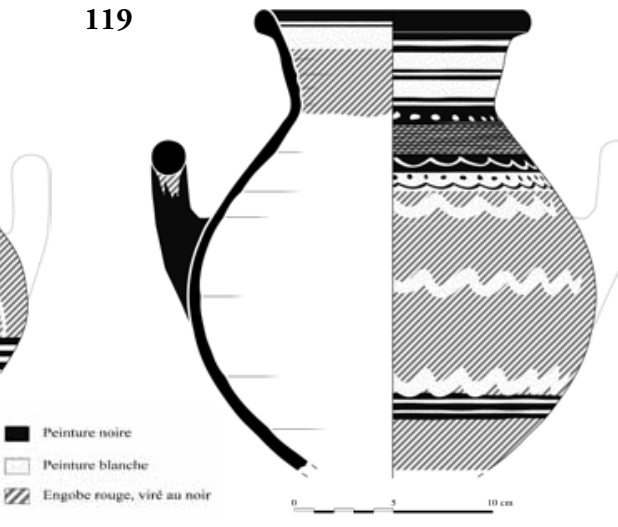

Fig. 116. - d/a.pw.23 (dessin EFA, J. Humbert).

Fig. 117. — d/a.bichr.r.3 (dessin EFA, J. Humbert).

Fig. 118. - d/a.bs.3 (dessin EFA, J. Humbert).

Fig. 119. - d/a.bs.2 (dessin EFA, J. Humbert).

BCH 133 (2009) 
120

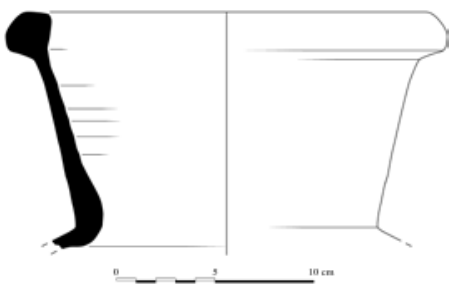

121

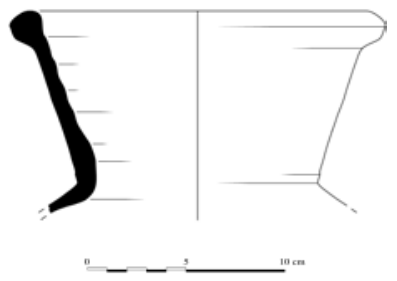

122

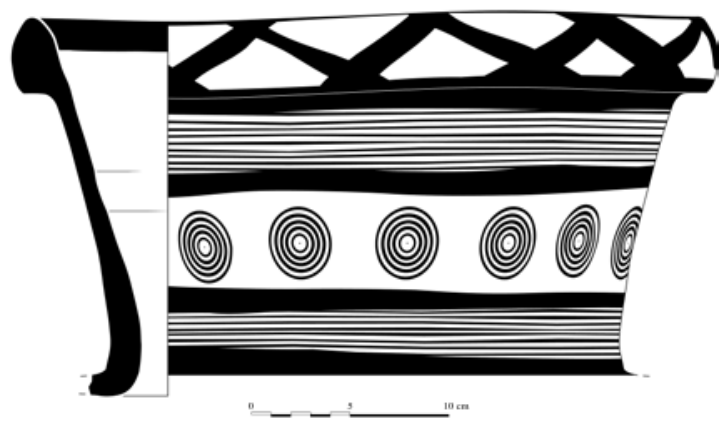

123

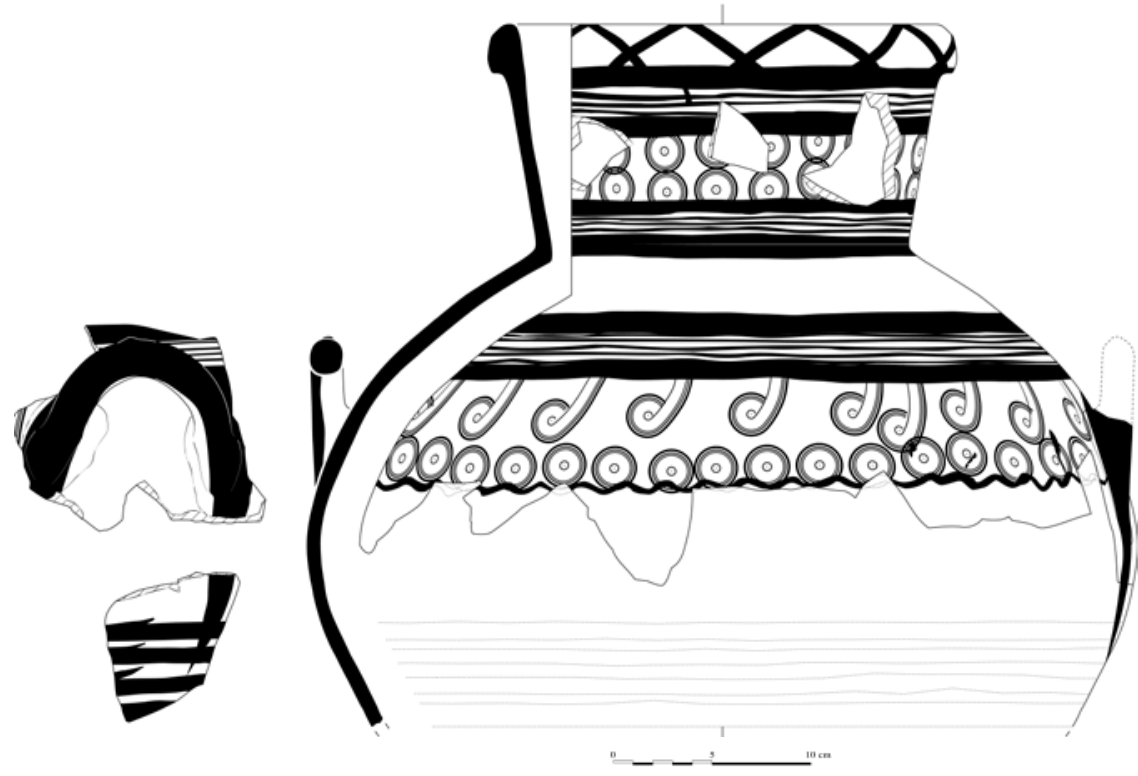

Fig. 120. - d/a.pw.21 (dessin EFA, J. Humbert).

Fig. 121. — d/a.pw.22 (dessin EFA, J. Humbert).

Fig. 122. — d/a.wp.5 (dessin EFA, J. Humbert).

Fig. 123. — d/a.wp.7 (dessin EFA, J. Humbert). 
124

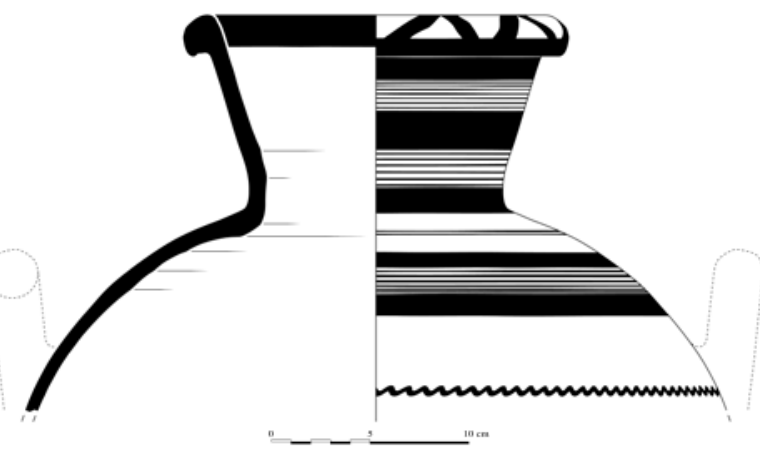

125

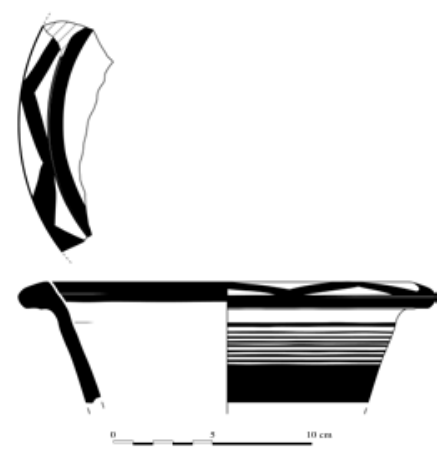

126
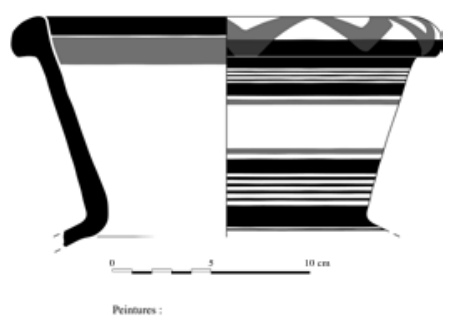

- Noir

Doupe

127
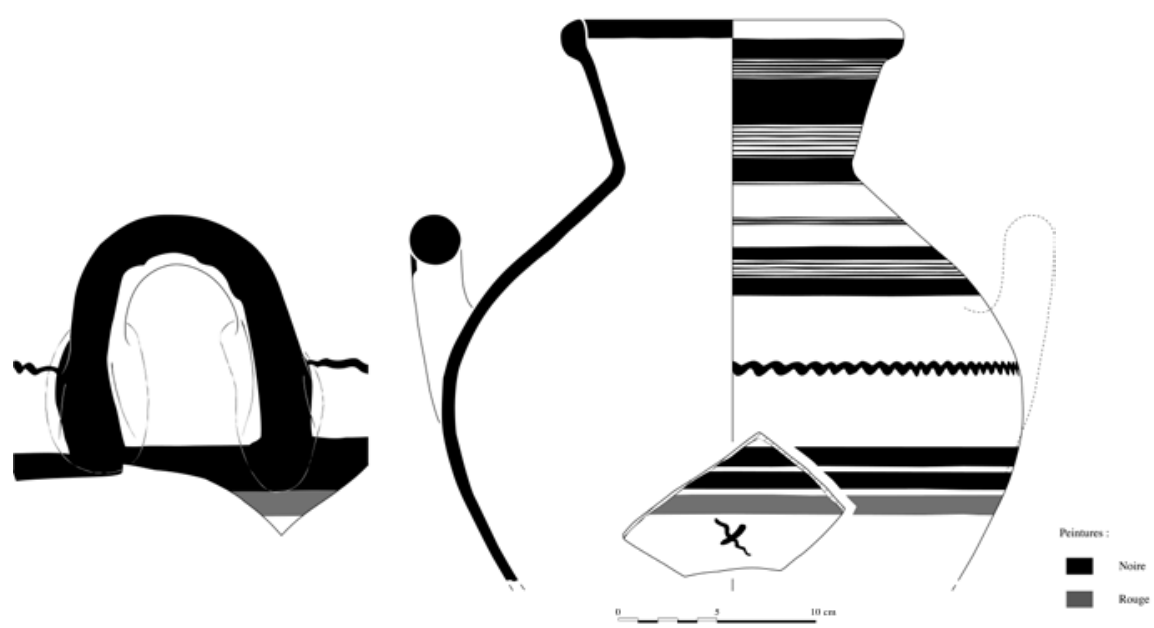

Fig. 124. - d/a.wp.8 (dessin EFA, J. Humbert).

Fig. 125. - d/a.wp.9 (dessin EFA, J. Humbert).

Fig. 126. — d/a.bichr.7 (dessin EFA, J. Humbert).

Fig. 127. — d/a.bichr.8 (dessin EFA, J. Humbert).

BCH 133 (2009) 
128

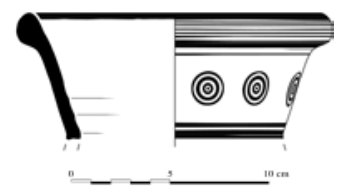

129
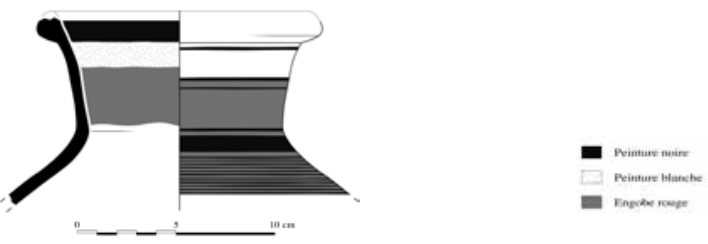

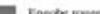

130

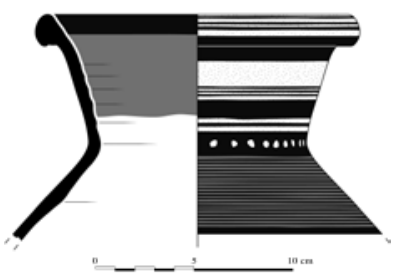

Peimuse neirs

Reintures blanche

- Eneres rom

131

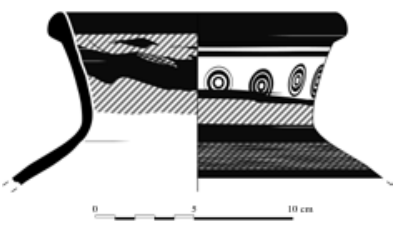

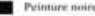

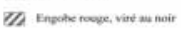

132

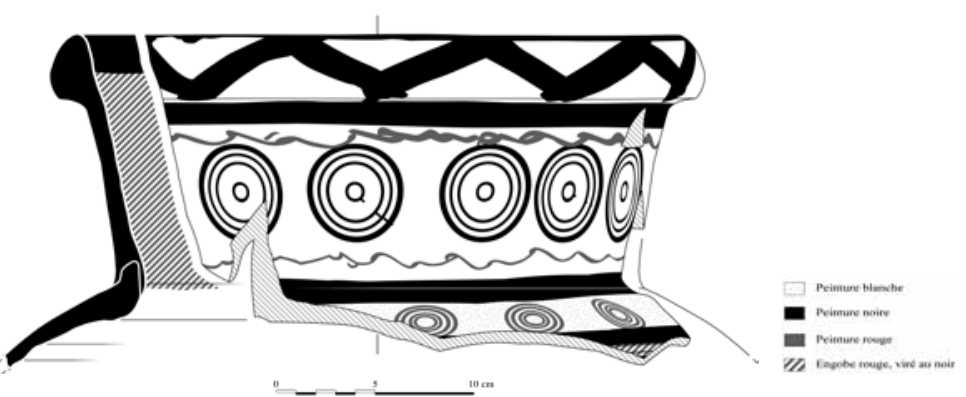

Fig. 128. - d/a.bor.4 (dessin EFA, J. Humbert).

Fig. 129. - d/a.bichr.r.5 (dessin EFA, J. Humbert).

Fig. 130. - d/a.bichr.r.6 (dessin EFA, J. Humbert).

Fig. 131. - d/a.bs.9 (dessin EFA, J. Humbert).

Fig. 132. — d/a.bs.10 (dessin EFA, J. Humbert). 
133 a

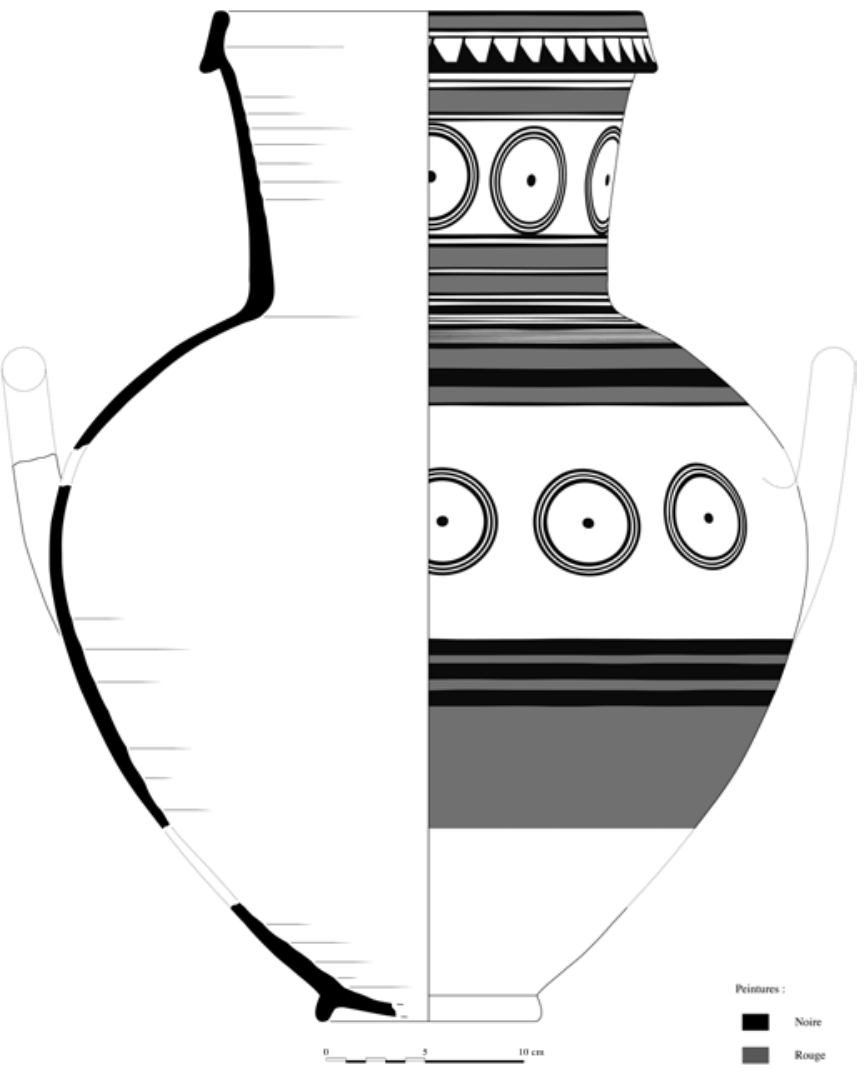

$133 \mathrm{~b}$

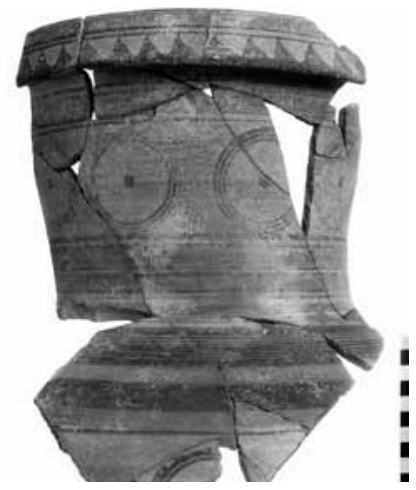

Fig. 133. — d/a.bichr.r.7 a. (dessin EFA, J. Humbert), b. (cl. EFA, Ph. Collet). 
134

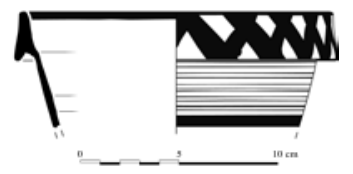

135
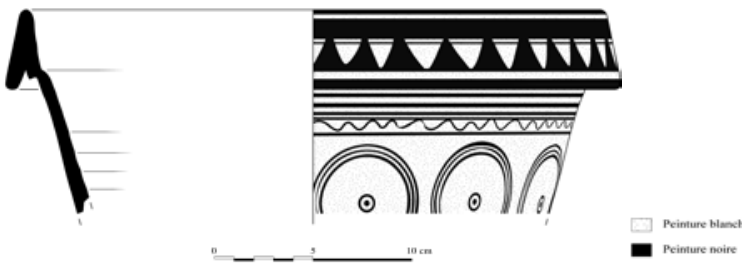

136
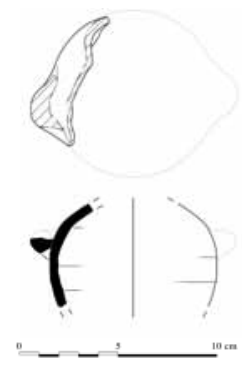

137 a
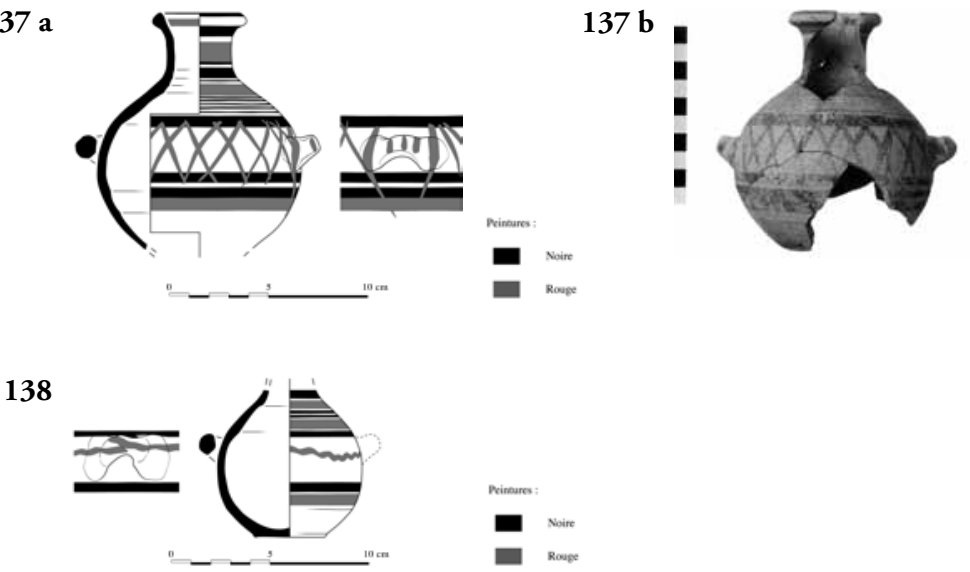

Fig. 134. - d/a.bs.7 (dessin EFA, J. Humbert).

Fig. 135. - d/a.bs.5 (dessin EFA, J. Humbert).

Fig. 136. - d/a.pw.24 (dessin EFA, J. Humbert).

Fig. 137. — d/a.bichr.19. a. (dessin EFA, J. Humbert), b. (cl. EFA, Ph. Collet).

Fig. 138. - d/a.bichr.20 (dessin EFA, J. Humbert). 
139

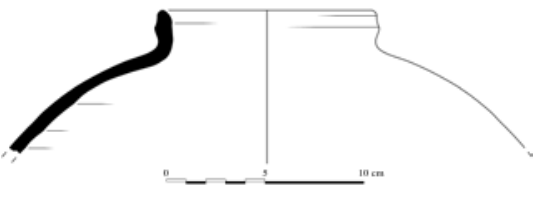

140

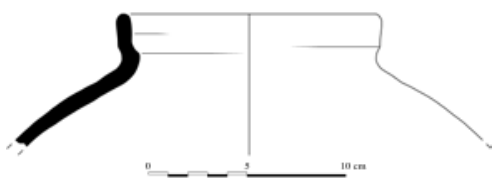

141

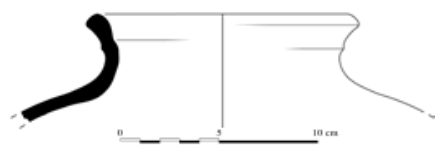

142

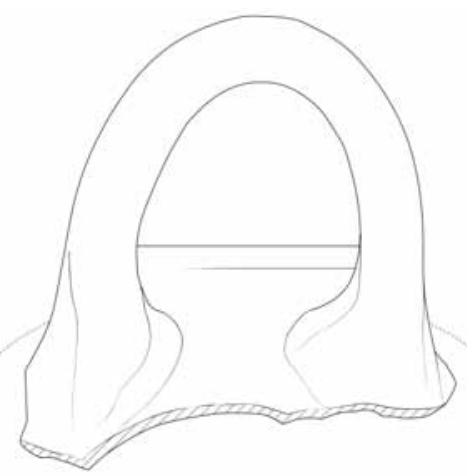

143

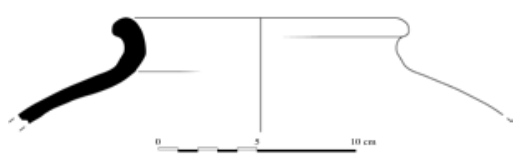

144

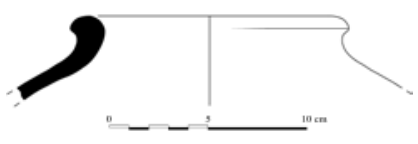

Fig. 139. - d/a.pw.7 (dessin EFA, J. Humbert).

Fig. 140. - d/a.pw.8 (dessin EFA, J. Humbert).

Fig. 141. — d/a.pw.9 (dessin EFA, J. Humbert).

Fig. 142. - d/a.pw.1 (dessin EFA, J. Humbert).

Fig. 143. - d/a.pw.2 (dessin EFA, J. Humbert).

Fig. 144. - d/a.pw.4 (dessin EFA, J. Humbert).

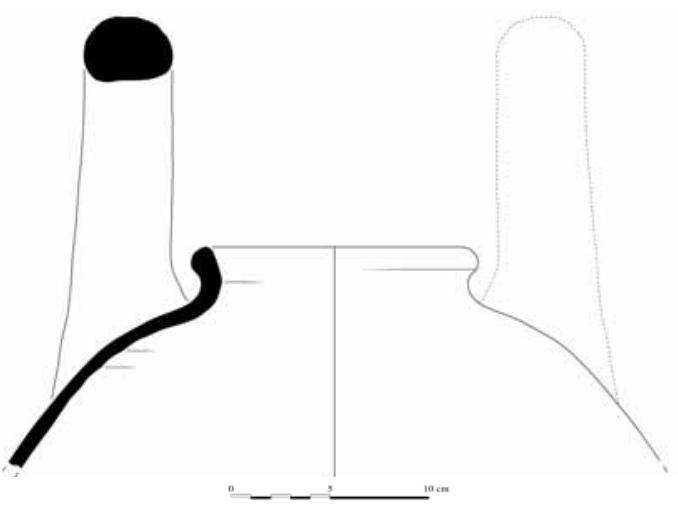

BCH 133 (2009) 
145

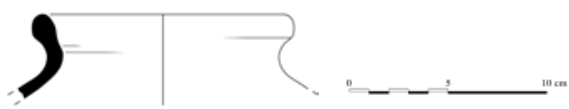

146
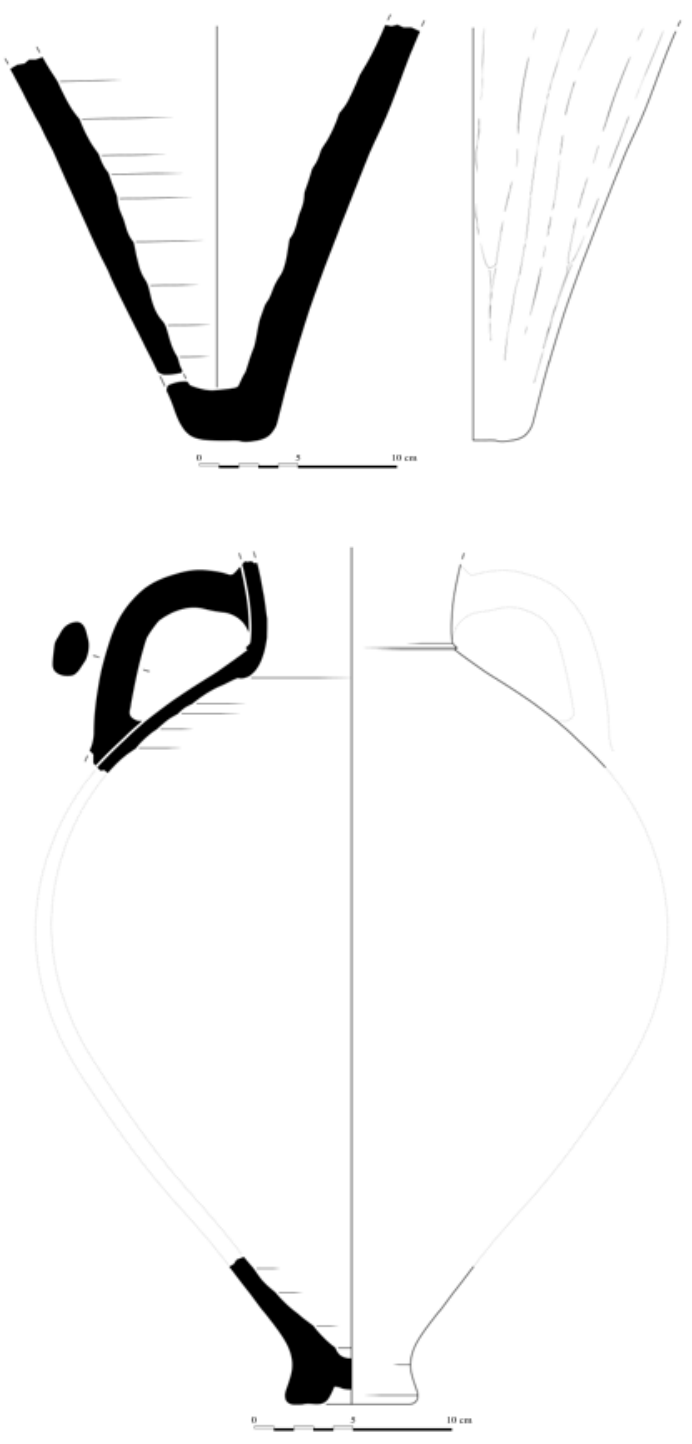

\section{8}

147
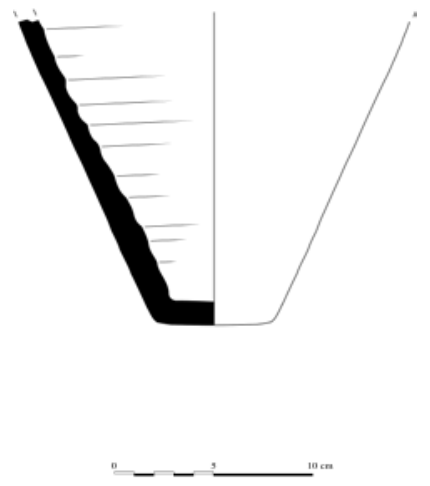

Fig. 145. - d/a.pw.6 (dessin EFA, J. Humbert).

Fig. 146 et 147. - Fonds d'amphores à anses de panier de type C.3 (dessin EFA, J. Humbert).

Fig. 148. - d/a.pw.16 (dessin EFA, J. Humbert). 
149

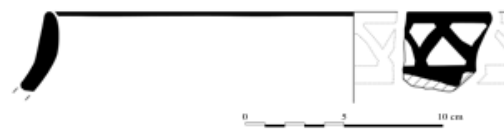

150

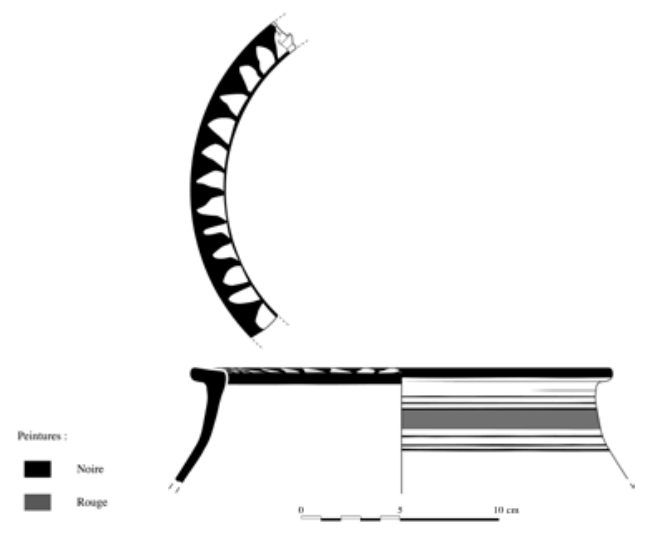

152

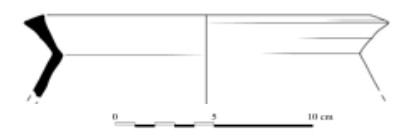

154

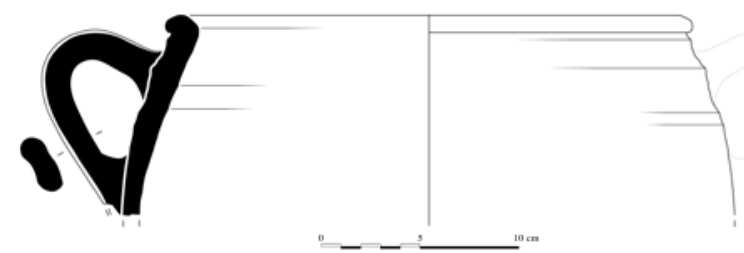

155
151

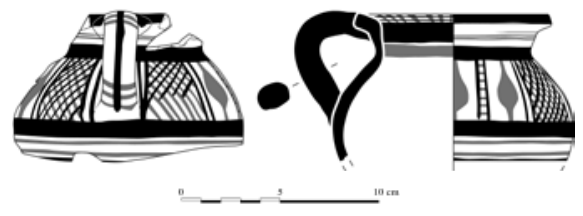

153

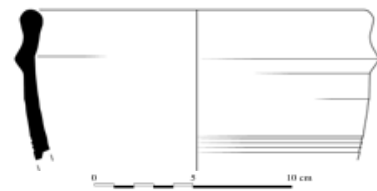

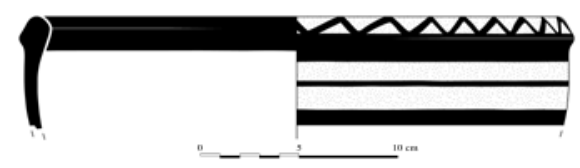

Fig. 149. - d/a.d.12 (dessin EFA, J. Humbert).

Fig. 150. — d/d.1 (dessin EFA, J. Humbert).

Fig. 151. — d/cr.bichr.3 (dessin EFA, J. Humbert).

Fig. 152. - d/d.2 (dessin EFA, J. Humbert).

Fig. 153. - d/b.pw.34 (dessin EFA, J. Humbert).

Fig. 154. - d/b.pw.37 (dessin EFA, J. Humbert).

Fig. 155. - d/d.3 (dessin EFA, J. Humbert).

BCH 133 (2009) 
156

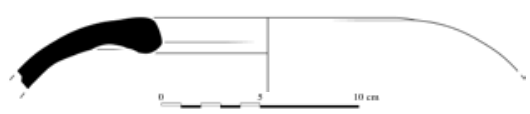

157

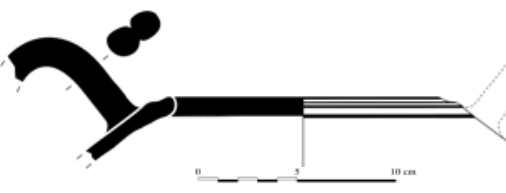

158

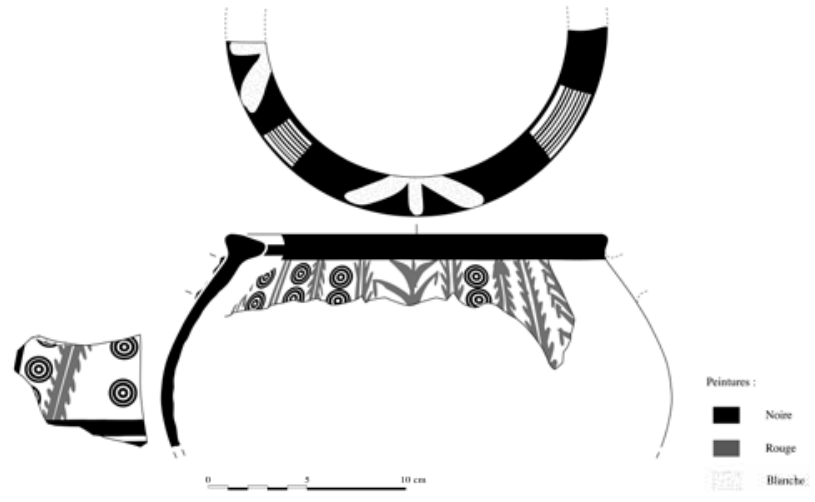

159
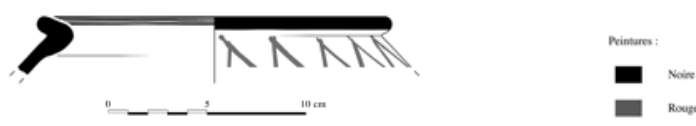

160
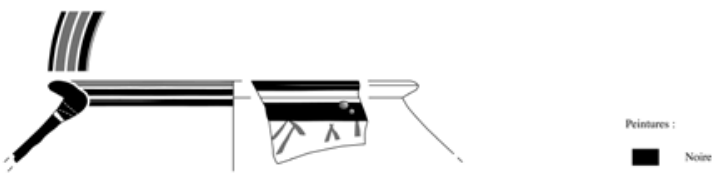

161

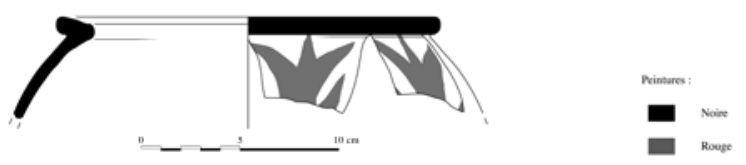

Fig. 156. - d/b.pw.36 (dessin EFA, J. Humbert).

Fig. 157. — d/d.4 (dessin EFA, J. Humbert).

Fig. 158. - d/d.6 (dessin EFA, J. Humbert).

Fig. 159. - d/d.7 (dessin EFA, J. Humbert).

Fig. 160. - d/d.8 (dessin EFA, J. Humbert).

Fig. 161. - d/d.9 (dessin EFA, J. Humbert). 
162
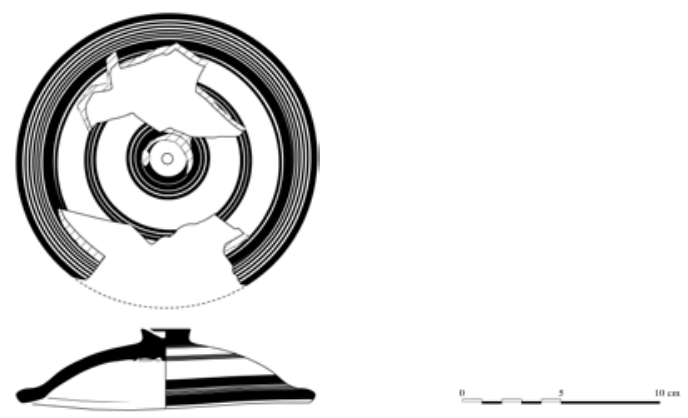

163
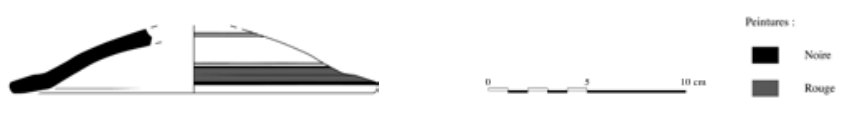

164
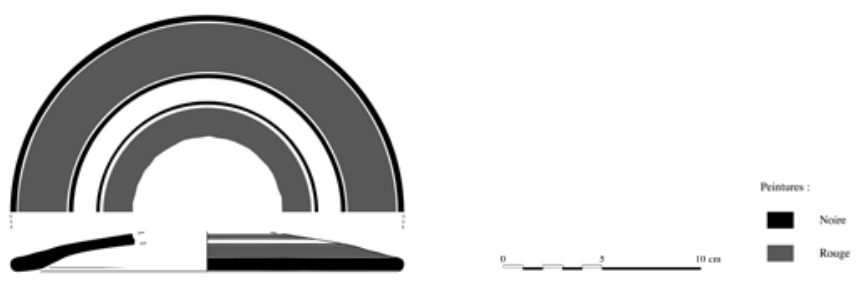

165

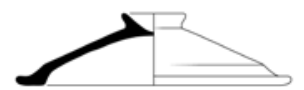

166
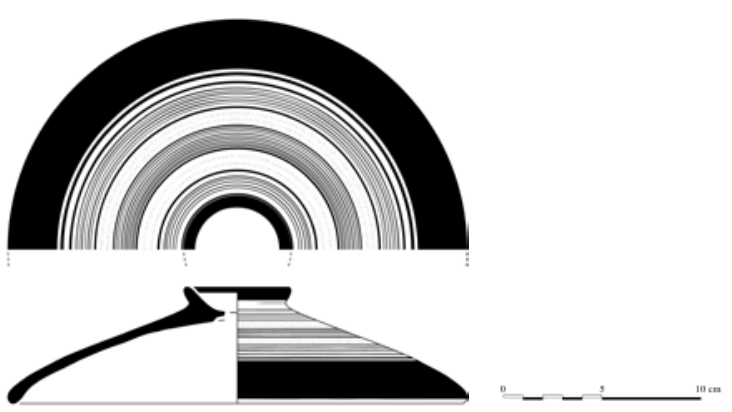

- Peinture noire

Peinture blanche

167
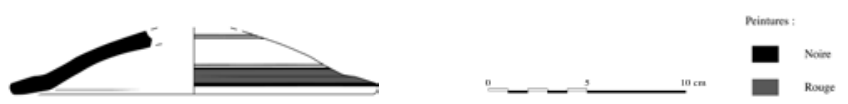

Fig. 162. - d/c.1 (dessin EFA, J. Humbert).

Fig. 163. - d/c.2 (dessin EFA, J. Humbert).

Fig. 164. - d/c.3 (dessin EFA, J. Humbert).

Fig. 165. - d/c.4 (dessin EFA, J. Humbert).

Fig. 166. - d/c.6 (dessin EFA, J. Humbert).

Fig. 167. - d/c.7 (dessin EFA, J. Humbert).

BCH 133 (2009) 
168

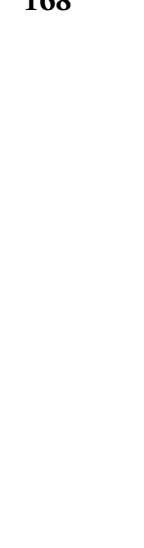

169
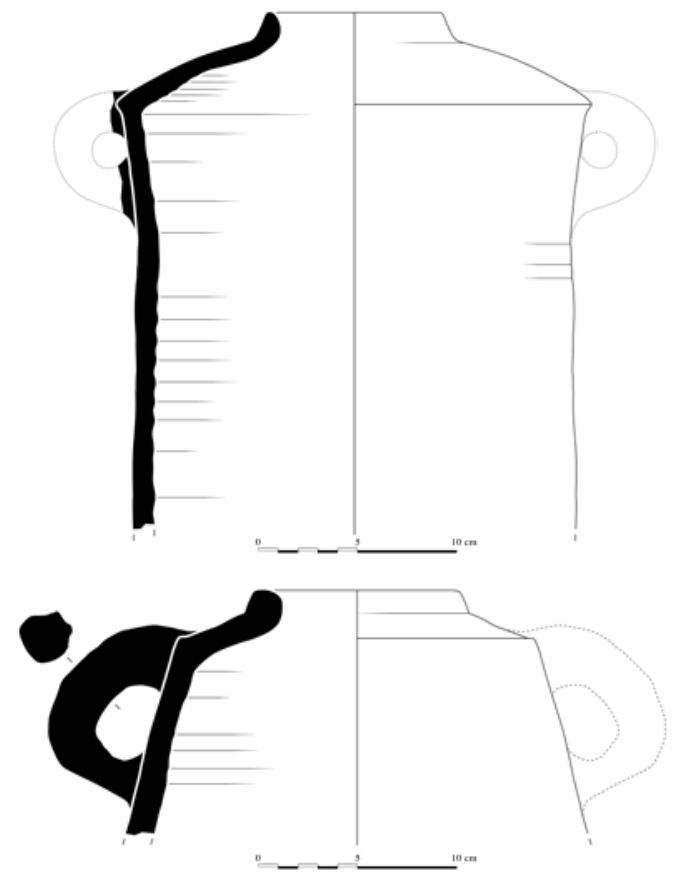

170

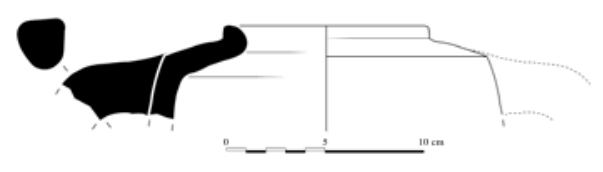

171

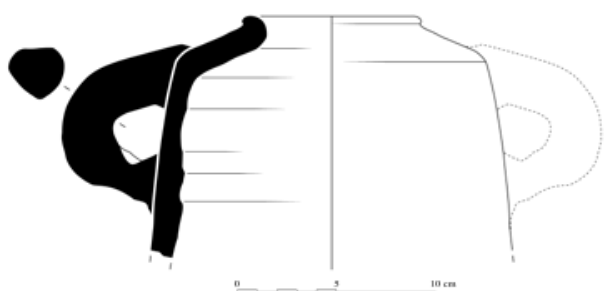

172

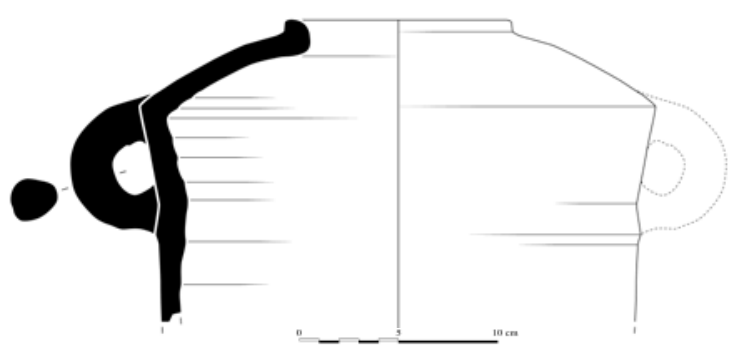

Fig. 168. - d/t.1 (dessin EFA, J. Humbert).

Fig. 169. - d/t.2 (dessin EFA, J. Humbert).

Fig. 170. - d/t.3 (dessin EFA, J. Humbert).

Fig. 171. - d/t.4 (dessin EFA, J. Humbert).

Fig. 172. - d/t.5 (dessin EFA, J. Humbert). 
173

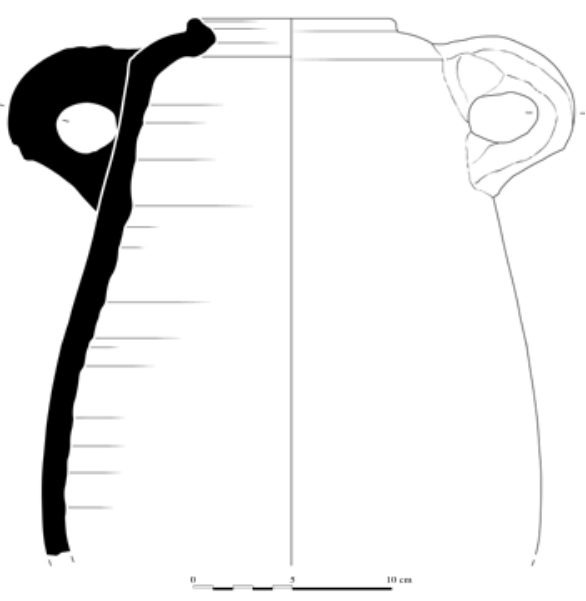

174

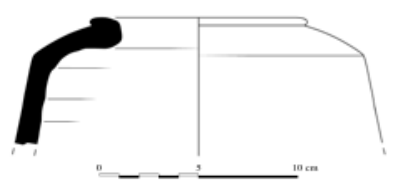

175

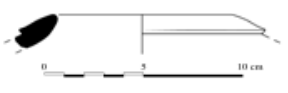

176

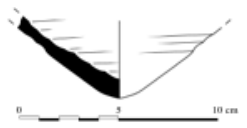

177

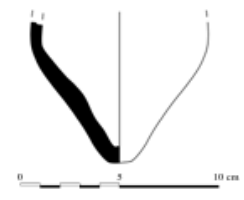

178

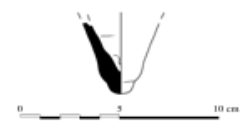

Fig. 173. $-\mathrm{d} / \mathrm{t} .6$ (dessin EFA, J. Humbert).

Fig. 174. — d/t.7 (dessin EFA, J. Humbert).

Fig. 175. - d/t.8 (dessin EFA, J. Humbert).

Fig. 176,177 et 178. — Fonds de jarres-torpilles (dessin EFA, J. Humbert).

BCH 133 (2009) 